\title{
Is Bismuth Really the 'Green' Metal? Exploring the Antimicrobial Activity and Cytotoxicity of Organobismuth-Thiolate Complexes
}

Liam J. Stephens Andrews*.*phil.andrews@monash.edu.

\section{Contents}

\section{Experimental Details}

Preparation of tetrazole thiolates and triazole thiolates

\section{Crystallography Details}

Tabulated data for crystallographic structures for select structures (Table S1 and Figure S1)

\section{Calculated Log P details}

C $\log$ P data for complexes 1-3 (Table S2)

\section{NMR Details}

${ }^{1} \mathrm{H}$ and ${ }^{13} \mathrm{C}$ NMR of tetrazole thiolate precursors (Figures S2-S13)

${ }^{1} \mathrm{H}$ and ${ }^{13} \mathrm{C}$ NMR of Bismuth complexes (1a-1l, 2a-2f and 3a-3l) (Figures S14-S73) 


\section{Preparation of tetrazole thiolates:}

GP5: Tetrazole thiolato synthesis: To a solution of $\mathrm{NaN}_{3}$ (3 equiv.) in deionised water (40 $\mathrm{mL}$ ) was added the substituted phenyl isothiocyanate (1 equiv.) portionally. The resulting solution was stirred at reflux for $5 \mathrm{~h}$ before being cooled to room temperate and washed with $2 \mathrm{x}$ ethyl acetate $(25 \mathrm{~mL})$ to remove the organic impurities. The remaining aqueous phase was first acidified to $\mathrm{pH} 2$ with concentrated $\mathrm{HCl}$, before the organics were then extracted with ethyl acetate $(3 \times 20 \mathrm{~mL})$, dried with $\mathrm{MgSO}_{4}$ and concentrated under vacuo to yield the desired compound as a solid.

GP6: Triazole thiolato synthesis: This reaction procedure was modelled from Youssef et al. ${ }^{1}$ and was further optimized using microwave irradiation producing improved yields of the resulting substituted phenyl carbothioamide derivatives and final thiols.

1-(p-Tolyl)-1H-tetrazole-5-thiol (a): Following GP5: $p$-tolyl isothiocyanate $(1.00 \mathrm{~g}, 6.70$ mmol) and $\mathrm{NaN}_{3}(1.31 \mathrm{~g}, 20.10 \mathrm{mmol})$ were stirred in $40 \mathrm{~mL}$ of deionised $\mathrm{H}_{2} \mathrm{O}$. The desired compound was obtained as a white solid without the need for further purification $(630 \mathrm{mg}$, 49\%). ${ }^{1} \mathrm{H}$ NMR (600 MHz, DMSO-d $d_{6} \delta 7.76-7.71(\mathrm{~m}, 2 \mathrm{H}), 7.43-7.38(\mathrm{~m}, 2 \mathrm{H}), 2.40(\mathrm{~s}$, $3 \mathrm{H}) .{ }^{13} \mathrm{C}$ NMR $\left(101 \mathrm{MHz}, \mathrm{DMSO}-d_{6}\right) \delta 163.6,139.5,131.5,129.7,124.4,20.8$. IR (film, $\mathrm{cm}^{-}$ $\left.{ }^{1}\right): v=3040\left(\mathrm{C}-\mathrm{H}_{\mathrm{AR}}\right), 2551(\mathrm{~S}-\mathrm{H})$. Melting point: $150-151{ }^{\circ} \mathrm{C}$. MS (ESI) calculated for $\mathrm{C}_{8} \mathrm{H}_{8} \mathrm{~N}_{4} \mathrm{~S}: 191.0469(\mathrm{M}-\mathrm{H})^{-}$.

1-(4-Dimethylamino)phenyl)-1H-tetrazole-5-thiol (b): Following GP5: $p$ dimethylaminophenyl isothiocyanate $(1.00 \mathrm{~g}, 6.00 \mathrm{mmol})$ and $\mathrm{NaN}_{3}(1.20 \mathrm{~g}, 18.00 \mathrm{mmol})$ were stirred in $40 \mathrm{~mL}$ of deionised $\mathrm{H}_{2} \mathrm{O}$. The desired compound was obtained as a white solid without the need for further purification (590 mg, 43\%). ${ }^{1} \mathrm{H}$ NMR (400 MHz, DMSO- $\left.d_{6}\right) \delta 7.59-7.54$ $(\mathrm{m}, 2 \mathrm{H}), 6.84(\mathrm{~d}, J=9.1 \mathrm{~Hz}, 2 \mathrm{H}), 2.99(\mathrm{~s}, 6 \mathrm{H}) .{ }^{13} \mathrm{C}$ NMR $\left(101 \mathrm{MHz}, \mathrm{DMSO}-d_{6}\right) \delta 150.7,125.6$, 122.4, 111.6, 40.0. IR (film, $\left.\mathrm{cm}^{-1}\right): v=3038\left(\mathrm{C}-\mathrm{H}_{\mathrm{AR}}\right), 2553(\mathrm{~S}-\mathrm{H}), 1348(\mathrm{C}-\mathrm{N})$. Melting point: $182{ }^{\circ} \mathrm{C}$. MS (ESI) calculated for $\mathrm{C}_{9} \mathrm{H}_{11} \mathrm{~N}_{5} \mathrm{~S}: 220.0735(\mathrm{M}-\mathrm{H})^{-}$.

1-Mesityl-1H-tetrazole-5-thiol (c): Following GP5: 2,4,6-trimethylphenyl isothiocyanate $(1.00 \mathrm{~g}, 5.64 \mathrm{mmol})$ and $\mathrm{NaN}_{3}(1.10 \mathrm{~g}, 16.92 \mathrm{mmol})$ were stirred in $40 \mathrm{~mL}$ of deionised $\mathrm{H}_{2} \mathrm{O}$. The desired compound was obtained as a white solid without the need for further purification (900 mg, 73\%). ${ }^{1} \mathrm{H}$ NMR (600 MHz, DMSO- $\left.d_{6}\right) \delta 7.11(\mathrm{~s}, 2 \mathrm{H}), 2.33(\mathrm{~s}, 3 \mathrm{H}), 1.95(\mathrm{~s}, 6 \mathrm{H}) .{ }^{13} \mathrm{C}$ NMR $\left(101 \mathrm{MHz}\right.$, DMSO- $\left.d_{6}\right) \delta 140.5,135.7,129.1,129.0,20.7,17.0$. IR (film, $\left.\mathrm{cm}^{-1}\right): v=3023$ $\left(\mathrm{C}-\mathrm{H}_{\mathrm{AR}}\right), 2576(\mathrm{~S}-\mathrm{H})$. Melting point: $173^{\circ} \mathrm{C}$. MS (ESI) calculated for $\mathrm{C}_{10} \mathrm{H}_{12} \mathrm{~N}_{4} \mathrm{~S}: 219.0782(\mathrm{M}$ $-\mathrm{H})^{-}$.

1-(4-Chlorophenyl)-1H-tetrazole-5-thiol (d): Following GP5: $p$-chlorophenyl isothiocyanate $(1.00 \mathrm{~g}, 5.89 \mathrm{mmol})$ and $\mathrm{NaN}_{3}(1.15 \mathrm{~g}, 17.69 \mathrm{mmol})$ were stirred in $40 \mathrm{~mL}$ of deionised $\mathrm{H}_{2} \mathrm{O}$. The desired compound was obtained as a white solid without the need for further purification (690 mg, 54\%). ${ }^{1} \mathrm{H}$ NMR (600 MHz, DMSO- $\left.d_{6}\right) \delta 7.99-7.94(\mathrm{~m}, 2 \mathrm{H}), 7.71-7.67(\mathrm{~m}, 2 \mathrm{H}) .{ }^{13} \mathrm{C}$ NMR (101 MHz, DMSO- $\left.d_{6}\right) \delta 134.0,132.9,129.3,126.2$. IR (film, $\left.\mathrm{cm}^{-1}\right): v=2971\left(\mathrm{C}-\mathrm{H}_{\mathrm{AR}}\right)$, $2092\left(\mathrm{C}-\mathrm{H}_{\mathrm{AR}}\right), 2548(\mathrm{~S}-\mathrm{H})$. Melting point: $178{ }^{\circ} \mathrm{C}$. MS (ESI) calculated for $\mathrm{C}_{7} \mathrm{H}_{5} \mathrm{ClN}_{4} \mathrm{~S}$ : $210.9924(\mathrm{M}-\mathrm{H})^{-}$.

1-(4-Methoxyphenyl)-1H-tetrazole-5-thiol (e): Following GP5: $p$-methoxyphenyl isothiocyanate $(1.00 \mathrm{~g}, 6.05 \mathrm{mmol})$ and $\mathrm{NaN}_{3}(1.18 \mathrm{~g}, 18.15 \mathrm{mmol})$ were stirred in $40 \mathrm{of} \mathrm{mL}$ deionised $\mathrm{H}_{2} \mathrm{O}$. The desired compound was obtained as a white solid without the need for further purification $(830 \mathrm{mg}, 62 \%) .{ }^{1} \mathrm{H}$ NMR $\left(600 \mathrm{MHz}, \mathrm{DMSO}-d_{6}\right) 7.76-7.73(\mathrm{~m}, 2 \mathrm{H}), 7.16$ - 7.12 (m, 2H), 3.84 (s, 3H). ${ }^{13} \mathrm{C}$ NMR (101 MHz, DMSO-d $) \delta 163.8,159.9,126.7,126.3$, 
114.4, 55.6. IR (film, $\left.\mathrm{cm}^{-1}\right): v=3052\left(\mathrm{C}-\mathrm{H}_{\mathrm{AR}}\right), 2559$ (S-H), 1251 (C-O). Melting point: 166 ${ }^{\circ} \mathrm{C}$. MS (ESI) calculated for $\mathrm{C}_{8} \mathrm{~N}_{8} \mathrm{~N}_{4} \mathrm{OS}: 203.0418(\mathrm{M}-\mathrm{H})^{-}$.

1-(4-Nitrophenyl)-1H-tetrazole-5-thiol (f): Following GP5: $p$-nitrophenyl isothiocyanate $(1.00 \mathrm{~g}, 5.56 \mathrm{mmol})$ and $\mathrm{NaN}_{3}(1.08 \mathrm{~g}, 16.67 \mathrm{mmol})$ were stirred in $40 \mathrm{~mL}$ of deionised $\mathrm{H}_{2} \mathrm{O}$. The desired compound was obtained as a yellow solid without the need for further purification (640 mg, 52\%). ${ }^{1} \mathrm{H}$ NMR (600 MHz, DMSO- $\left.d_{6}\right) \delta 8.49-8.43(\mathrm{~m}, 2 \mathrm{H}), 8.39-8.32(\mathrm{~m}, 2 \mathrm{H}) .{ }^{13} \mathrm{C}$ NMR (101 MHz, DMSO-d $\left.d_{6}\right) 163.9,147.1,139.0,124.7,124.6$. IR (film, $\left.\mathrm{cm}^{-1}\right): v=2998(\mathrm{C}-$ $\left.\mathrm{H}_{\mathrm{AR}}\right), 1515(\mathrm{~N}-\mathrm{O})$. Melting point: $145^{\circ} \mathrm{C}$. MS (ESI) calculated for $\mathrm{C}_{7} \mathrm{H}_{5} \mathrm{~N}_{5} \mathrm{O}_{2} \mathrm{~S}: 222.0164(\mathrm{M}-$ $\mathrm{H})^{-}$.

Following GP6: All analytical data such as NMR spectra, melting point and IR were consistent with the literature reported values for compounds $\mathbf{g}-\mathbf{l} .^{1}$ 
Table S1. Crystallography data for complexes $1 \mathrm{a}, \mathbf{1 b}, \mathbf{1 d}, \mathbf{1 f}, \mathbf{1 g}, \mathbf{1 h}, \mathbf{1 k}, \mathbf{1 l}, \mathbf{2 c}$ and $\mathbf{2 d}$.

\begin{tabular}{|c|c|c|c|c|c|}
\hline & 1a & $1 \mathrm{~b}$ & 1d & 1f & $1 \mathrm{~g}$ \\
\hline $\begin{array}{l}\text { Chemical } \\
\text { Formula }\end{array}$ & $\mathrm{C}_{20} \mathrm{H}_{17} \mathrm{BiN}_{4} \mathrm{~S}$ & $\mathrm{C}_{21} \mathrm{H}_{20} \mathrm{BiN}_{5} \mathrm{~S}$ & $\mathrm{BiC}_{19} \mathrm{ClN}_{4} \mathrm{~S}$ & $\mathrm{C}_{19} \mathrm{H}_{14} \mathrm{BiN}_{5} \mathrm{O}_{2} \mathrm{~S}$ & $\mathrm{C}_{25} \mathrm{H}_{19} \mathrm{BiN}_{4} \mathrm{~S}$ \\
\hline $\mathbf{M}_{r}$ & 554.41 & 583.46 & 560.72 & 585.39 & 616.48 \\
\hline Temp (K) & 293 & 293 & $123.01(10)$ & $123.01(10)$ & $122.99(10)$ \\
\hline $\begin{array}{l}\text { Crystal } \\
\text { system, space } \\
\text { group }\end{array}$ & $\begin{array}{l}\text { Orthorhombic, } \\
\mathrm{P} 2_{1} 2_{1} 2_{1}\end{array}$ & $\begin{array}{l}\text { Orthorhombic, } \\
\mathrm{P} 2_{1} 2_{1} 2_{1}\end{array}$ & $\begin{array}{l}\text { Orthorhombic, } \\
\mathrm{P} 2_{1} 2_{1} 2_{1}\end{array}$ & $\begin{array}{l}\text { Orthorhombic, } \\
\mathrm{P} 2_{1} 2_{1} 2_{1}\end{array}$ & $\begin{array}{l}\text { Monoclinic, } \\
\mathrm{P} 2_{1} / \mathrm{c}\end{array}$ \\
\hline $\mathbf{a}, \mathbf{b}, \mathbf{c}(\AA)$ & $\begin{array}{l}8.2212(2) \\
12.7038(3) \\
18.7564(6)\end{array}$ & $\begin{array}{l}8.5610(2) \\
12.7275(4) \\
18.7377(6)\end{array}$ & $\begin{array}{l}8.2564(3) \\
12.6860(4) \\
18.5257(6)\end{array}$ & $\begin{array}{l}8.3203(2) \\
12.6779(3) \\
18.1973(3)\end{array}$ & $\begin{array}{l}6.0666(2) \\
20.4595(8) \\
18.3557(7)\end{array}$ \\
\hline $\mathbf{V}$ & $2041.2(2)$ & $2041.66(10)$ & 1940.39(11) & $1919.52(7)$ & $2250.20(15)$ \\
\hline $\mathbf{Z}$ & 5 & 4 & 4 & 4 & 4 \\
\hline $\mathbf{T}_{\min }, \mathbf{T}_{\max }$ & $0.211,0.830$ & $0.44142,1.00000$ & $0.45125,1.00000$ & $0.26872,1.00000$ & $0.38752,1.00000$ \\
\hline $\mathbf{R}_{\text {int }}$ & 0.0303 & 0.0450 & 0.0483 & 0.094 & 0.0330 \\
\hline $\mathbf{R}_{1}$ & 0.0290 & 0.0322 & 0.0312 & 0.0682 & 0.0279 \\
\hline $\mathbf{W} \mathbf{r}^{2}$ & 0.0729 & 0.0519 & 0.0662 & 0.1843 & 0.0533 \\
\hline $\begin{array}{l}\text { No. of } \\
\text { reflections }\end{array}$ & 5771 & 16336 & 13478 & 8716 & 19415 \\
\hline $\begin{array}{l}\text { No. of } \\
\text { Parameters }\end{array}$ & 113 & 255 & 235 & 253 & 280 \\
\hline
\end{tabular}

Table $\mathbf{S 1}$ continued.

\begin{tabular}{|c|c|c|c|c|c|}
\hline & 1h & $1 \mathrm{k}$ & 11 & $2 \mathrm{c}$ & $2 d$ \\
\hline $\begin{array}{l}\text { Chemical } \\
\text { Formula }\end{array}$ & $\mathrm{C}_{29.5} \mathrm{H}_{27} \mathrm{BiN}_{4} \mathrm{OS}$ & $\mathrm{C}_{25} \mathrm{H}_{20} \mathrm{BiFN}_{4} \mathrm{OS}$ & $\mathrm{C}_{27} \mathrm{H}_{25} \mathrm{BiN}_{4} \mathrm{OS}$ & $\mathrm{C}_{30} \mathrm{H}_{39} \mathrm{BiN}_{8} \mathrm{O}_{2} \mathrm{~S}_{4}$ & $\mathrm{C}_{22} \mathrm{H}_{19} \mathrm{BiCl}_{2} \mathrm{~N}_{8} \mathrm{OS}_{3}$ \\
\hline $\mathbf{M}_{r}$ & 694.59 & 652.49 & 662.55 & 880.91 & 787.51 \\
\hline Temp (K) & $123.00(10)$ & $123.00(10)$ & $123.00(10)$ & $123.00(10)$ & $123.00(10)$ \\
\hline $\begin{array}{l}\text { Crystal } \\
\text { system, space } \\
\text { group }\end{array}$ & $\begin{array}{l}\text { Triclinic, } \\
\text { P-1 }\end{array}$ & Monoclinic, $\mathrm{P} 2{ }_{1} / \mathrm{c}$ & Monoclinic, $\mathrm{P}_{1} / \mathrm{c}$ & $\begin{array}{l}\text { Monoclinic, } \\
\mathrm{P} 2_{1} / \mathrm{n}\end{array}$ & Triclinic, P-1 \\
\hline $\mathbf{a}, \mathbf{b}, \mathbf{c}(\AA)$ & $\begin{array}{l}9.9428(2) \\
10.8585(2) \\
13.1690(3)\end{array}$ & $\begin{array}{l}11.1721(3) \\
9.6087(3) \\
22.2528(6)\end{array}$ & $\begin{array}{l}11.1369(4) \\
9.5326(4) \\
24.4057(11)\end{array}$ & $\begin{array}{l}8.60530(10) \\
23.5304(4) \\
17.7290(3)\end{array}$ & $\begin{array}{l}16.4020(2) \\
17.2623(3) \\
19.9603(3)\end{array}$ \\
\hline $\mathbf{V}$ & $1362.12(5)$ & $2313.85(12)$ & $2523.09(18)$ & $3587.97(10)$ & $5432.60(15)$ \\
\hline $\mathbf{Z}$ & 2 & 4 & 4 & 4 & 8 \\
\hline $\mathbf{T}_{\min }, \mathbf{T}_{\max }$ & $0.67442,1.00000$ & $0.46472,1.00000$ & $0.34511,1.00000$ & $0.59655,1.00000$ & $0.73902,1.00000$ \\
\hline $\mathbf{R}_{\text {int }}$ & 0.0673 & 0.0500 & 0.0550 & 0.0889 & 0.0593 \\
\hline $\mathbf{R}_{1}$ & 0.0348 & 0.0271 & 0.0503 & 0.0433 & 0.0427 \\
\hline $\mathbf{W r ^ { 2 }}$ & 0.0633 & 0.0528 & 0.1043 & 0.1074 & 0.0936 \\
\hline $\begin{array}{l}\text { No. of } \\
\text { reflections }\end{array}$ & 31251 & 29090 & 16783 & 27944 & 95746 \\
\hline
\end{tabular}




\begin{tabular}{|l|l|l|l|l|l|}
\hline $\begin{array}{l}\text { No. of } \\
\text { Parameters }\end{array}$ & 364 & 299 & 310 & 434 & 1369 \\
\hline
\end{tabular}

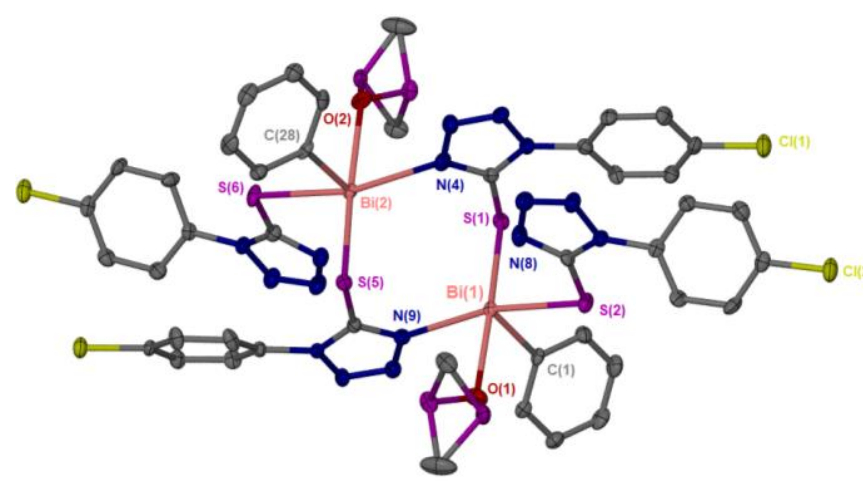

Figure S1. Molecular structure of complex 2d highlighting disorder of DMSO

Table S2. C $\log$ P values of complexes 1,2 and 3. Log P values were predicted using the

\begin{tabular}{|c|c|c|c|}
\hline \multicolumn{4}{|c|}{ ChemDraw 18.1 software. } \\
\hline Complex number & $C \log P$ & Complex number & $C \log P$ \\
\hline $1 a$ & 5.745 & $1 \mathrm{~g}$ & 5.334 \\
\hline 1b & 5.411 & 1h & 5.833 \\
\hline 1c & 6.143 & $1 \mathbf{i}$ & 5.253 \\
\hline $1 d$ & 5.959 & $\mathbf{1 j}$ & 6.047 \\
\hline $1 e$ & 5.165 & $1 k$ & 5.047 \\
\hline $1 f$ & 4.989 & 11 & 6.332 \\
\hline $2 a$ & 6.113 & & \\
\hline $2 b$ & 5.445 & & \\
\hline $2 c$ & 6.909 & & \\
\hline $2 d$ & 6.541 & & \\
\hline $2 e$ & 4.953 & & \\
\hline $2 f$ & 4.601 & & \\
\hline $3 a$ & 6.481 & $3 g$ & 5.248 \\
\hline $3 \mathbf{b}$ & 5.479 & $3 \mathrm{~h}$ & 6.745 \\
\hline $3 c$ & 7.675 & $3 \mathbf{i}$ & 5.005 \\
\hline $3 d$ & 7.123 & $3 \mathbf{j}$ & 7.387 \\
\hline $3 e$ & 4.741 & $3 k$ & 5.677 \\
\hline $3 f$ & 4.213 & 31 & 8.242 \\
\hline
\end{tabular}




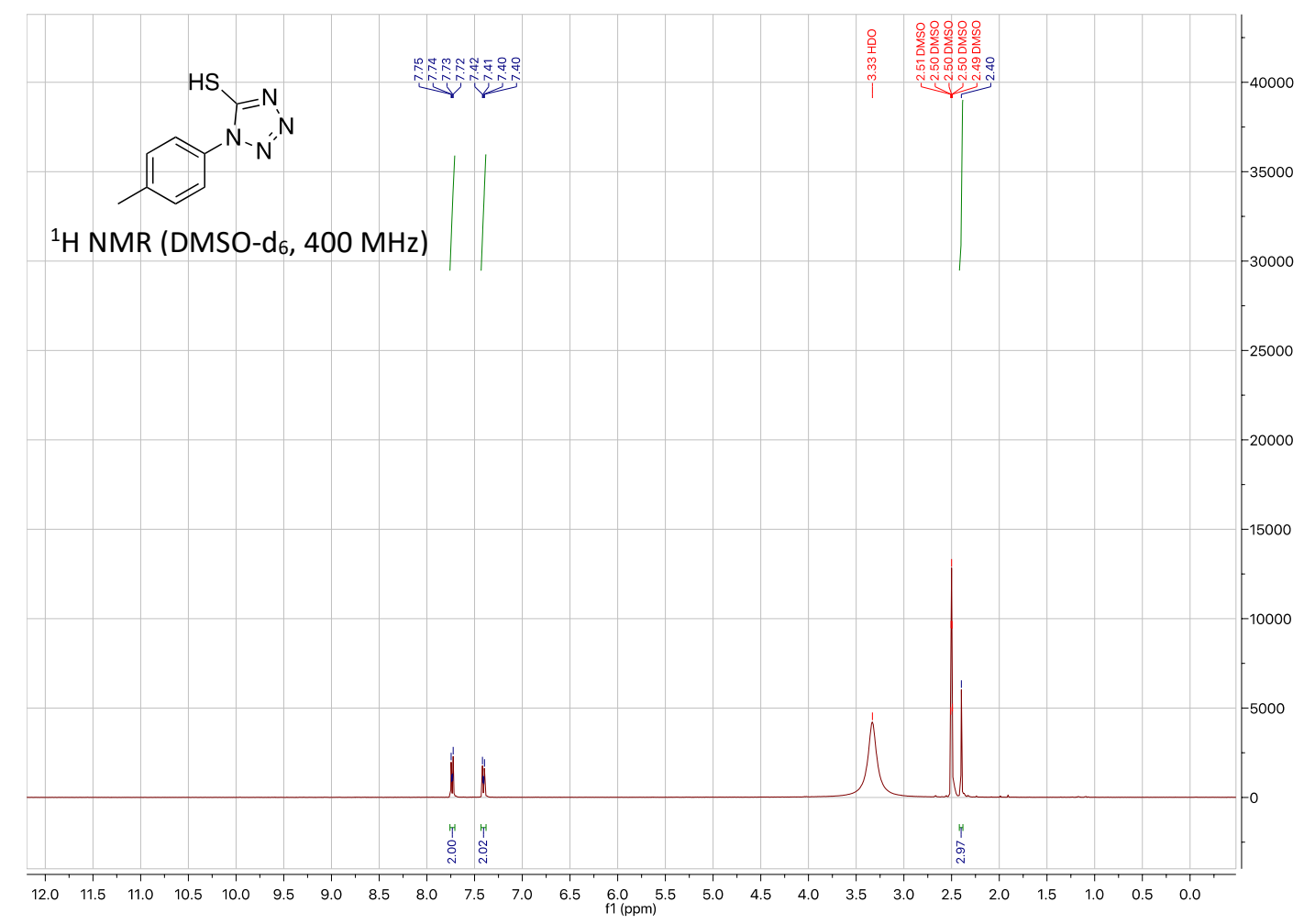

Figure S2. ${ }^{1} \mathrm{H}$ NMR of 1-(p-tolyl)-1H-tetrazole-5-thiol a (DMSO-d $\left.{ }_{6}\right)$

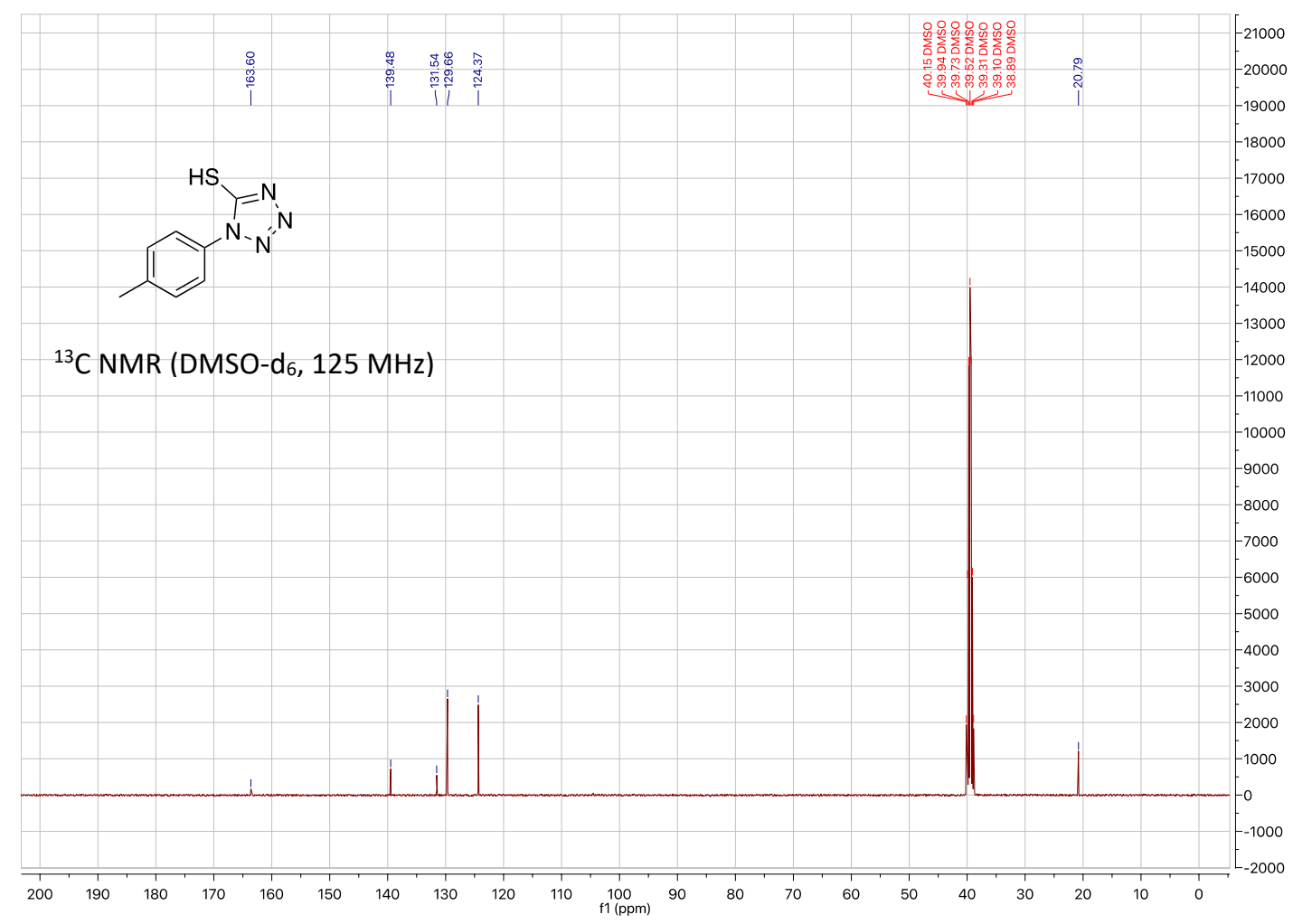

Figure S3. ${ }^{13} \mathrm{C}$ NMR of 1 -(p-tolyl)-1H-tetrazole-5-thiol a (DMSO-d 6 ) 


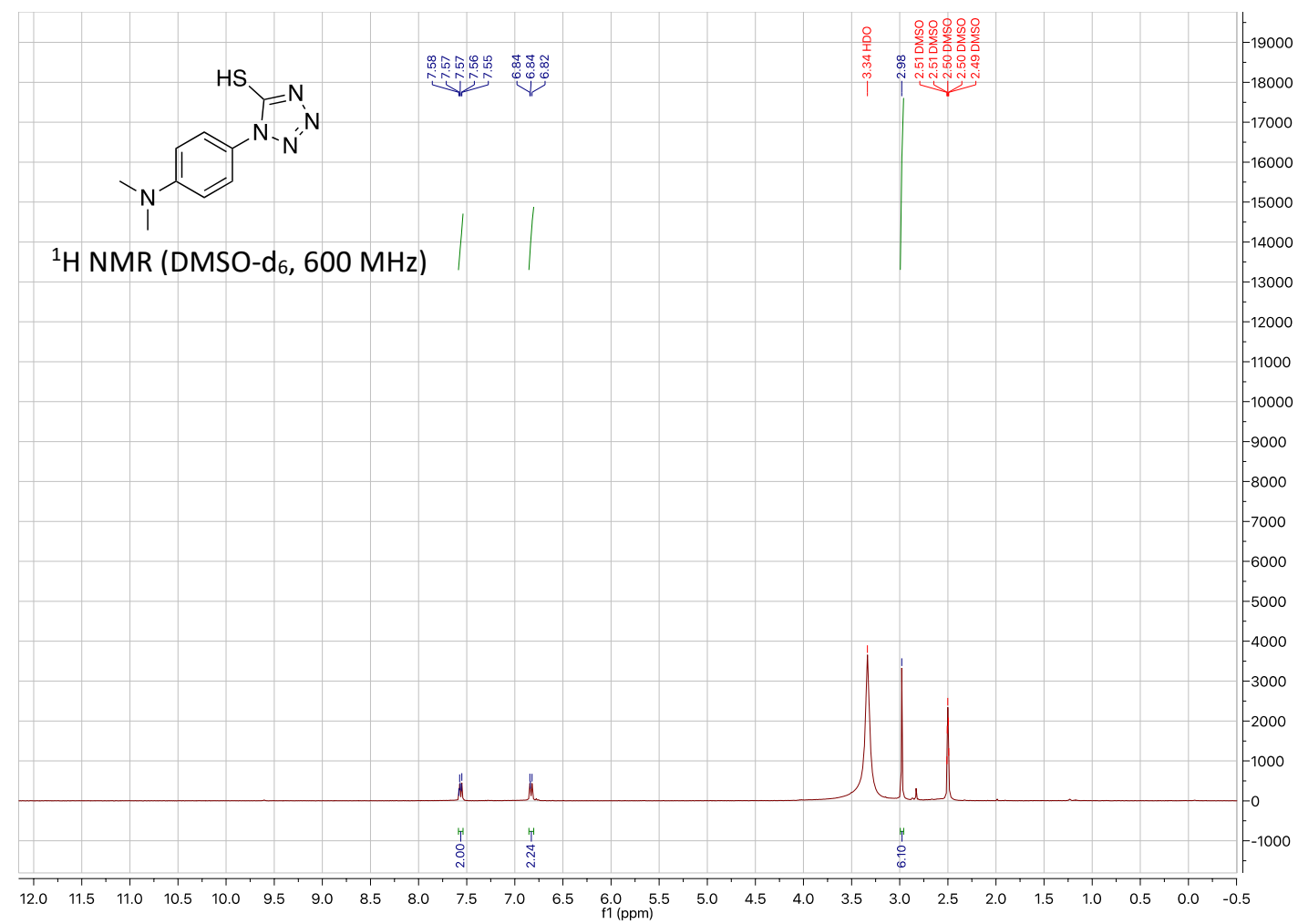

Figure S4. ${ }^{1} \mathrm{H}$ NMR of $p$-dimethylaminophenyl-1H-tetrazole-5-thiol b (DMSO-d ${ }_{6}$ )

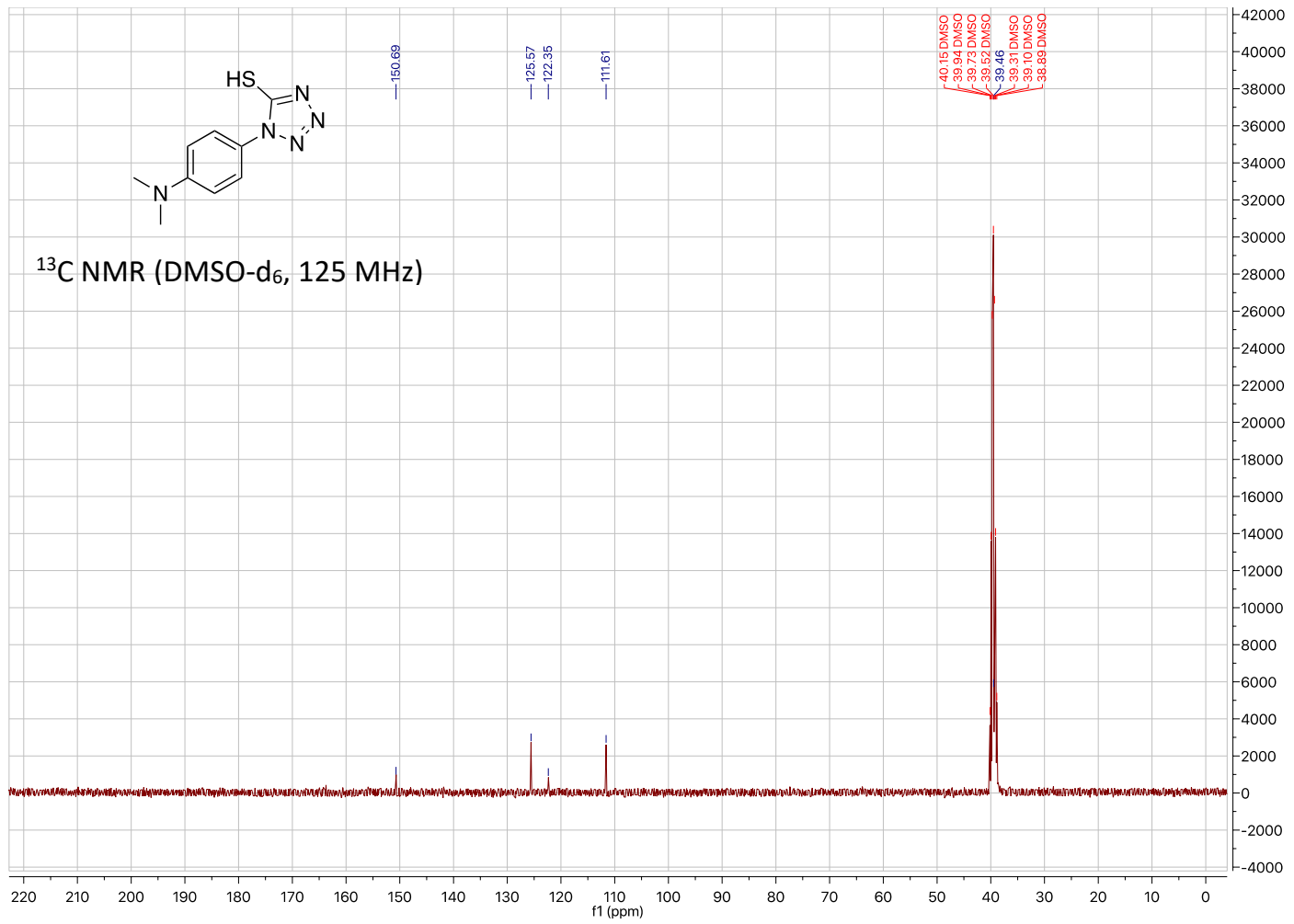

Figure S5. ${ }^{13} \mathrm{C}$ NMR of $p$-dimethylaminophenyl-1H-tetrazole-5-thiol b (DMSO-d ${ }_{6}$ ) 


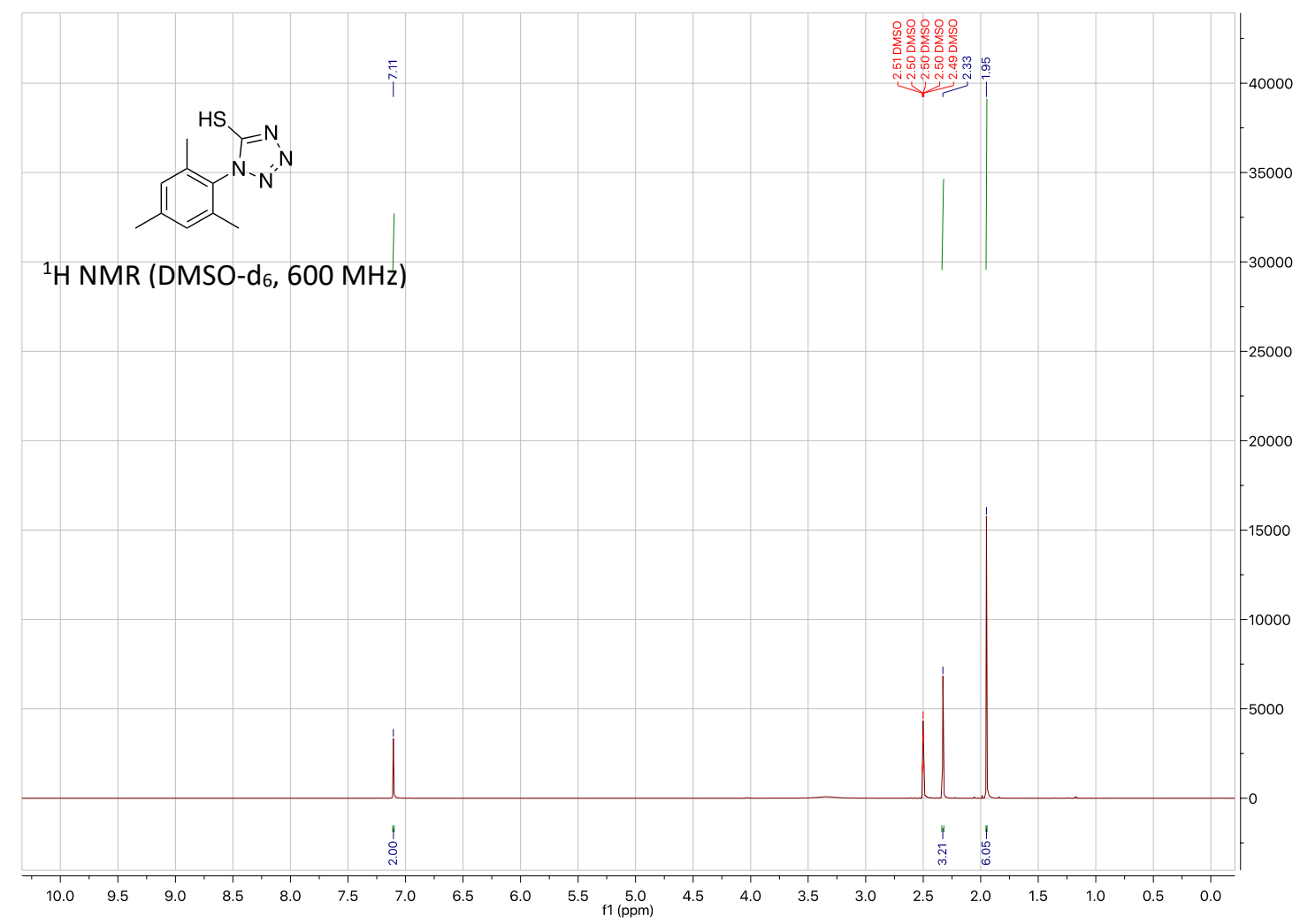

Figure S6. ${ }^{1} \mathrm{H}$ NMR of 1-mesityl-1H-tetrazole-5-thiol c (DMSO-d 6 )

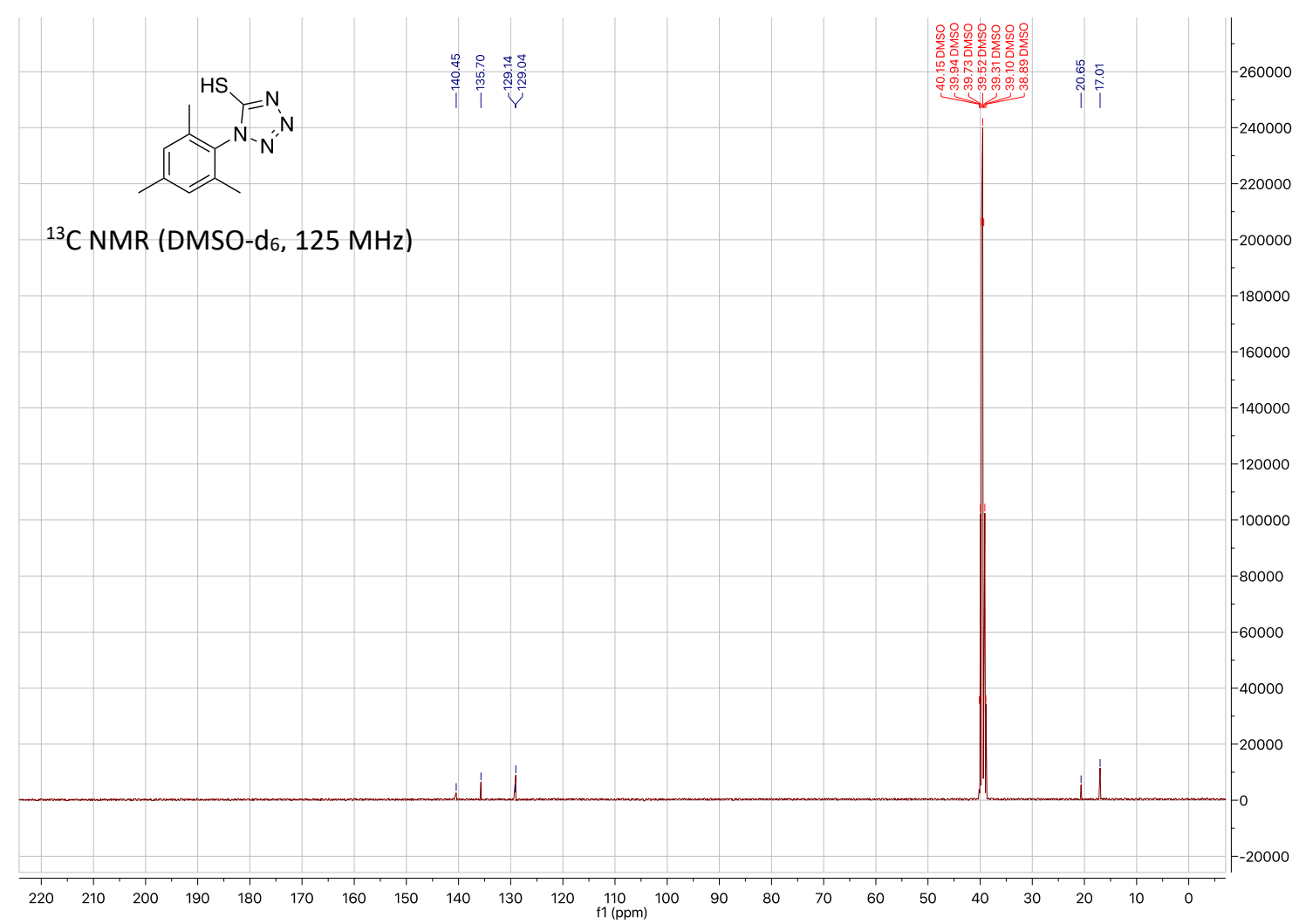

Figure S7. ${ }^{13} \mathrm{C}$ NMR of 1-mesityl-1H-tetrazole-5-thiol c (DMSO-d $\left.\mathrm{d}_{6}\right)$ 


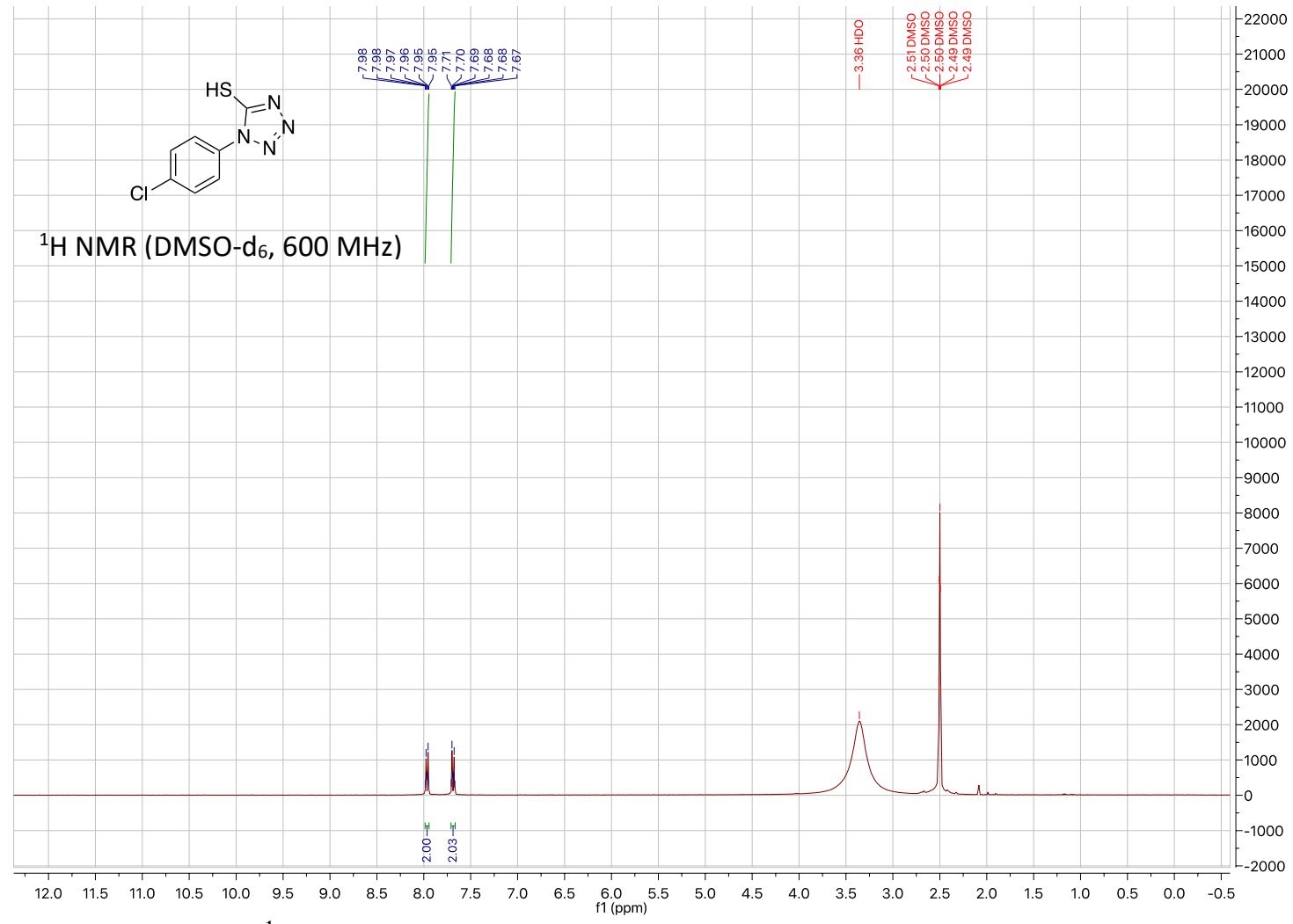

Figure S8. ${ }^{1} \mathrm{H}$ NMR of 1-(4-chlorophenyl)-1H-tetrazole-5-thiol d (DMSO-d $\left.\mathrm{d}_{6}\right)$

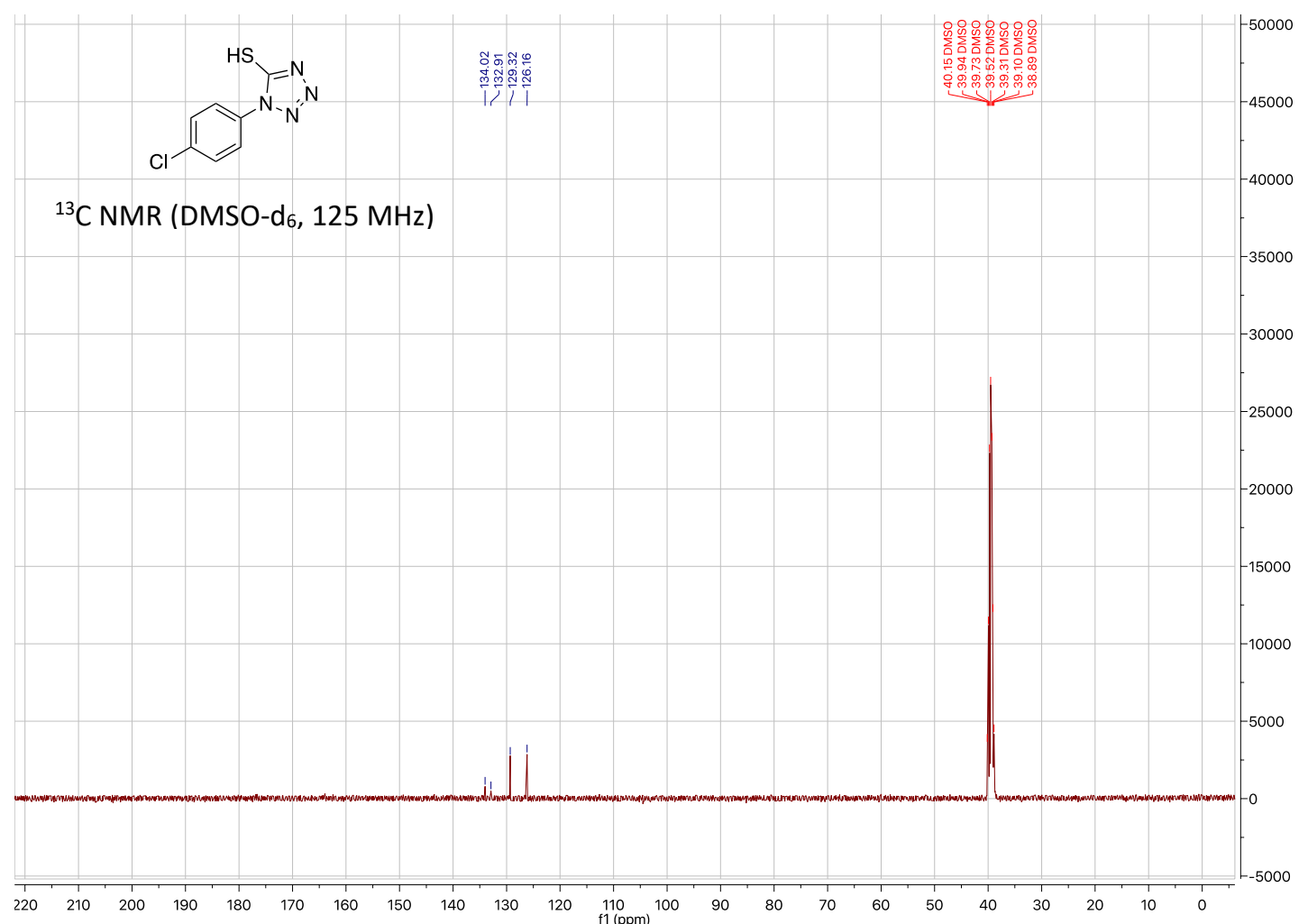

Figure S9. ${ }^{13} \mathrm{C}$ NMR of 1-(4-chlorophenyl)-1H-tetrazole-5-thiol d (DMSO-d $\left.{ }_{6}\right)$ 


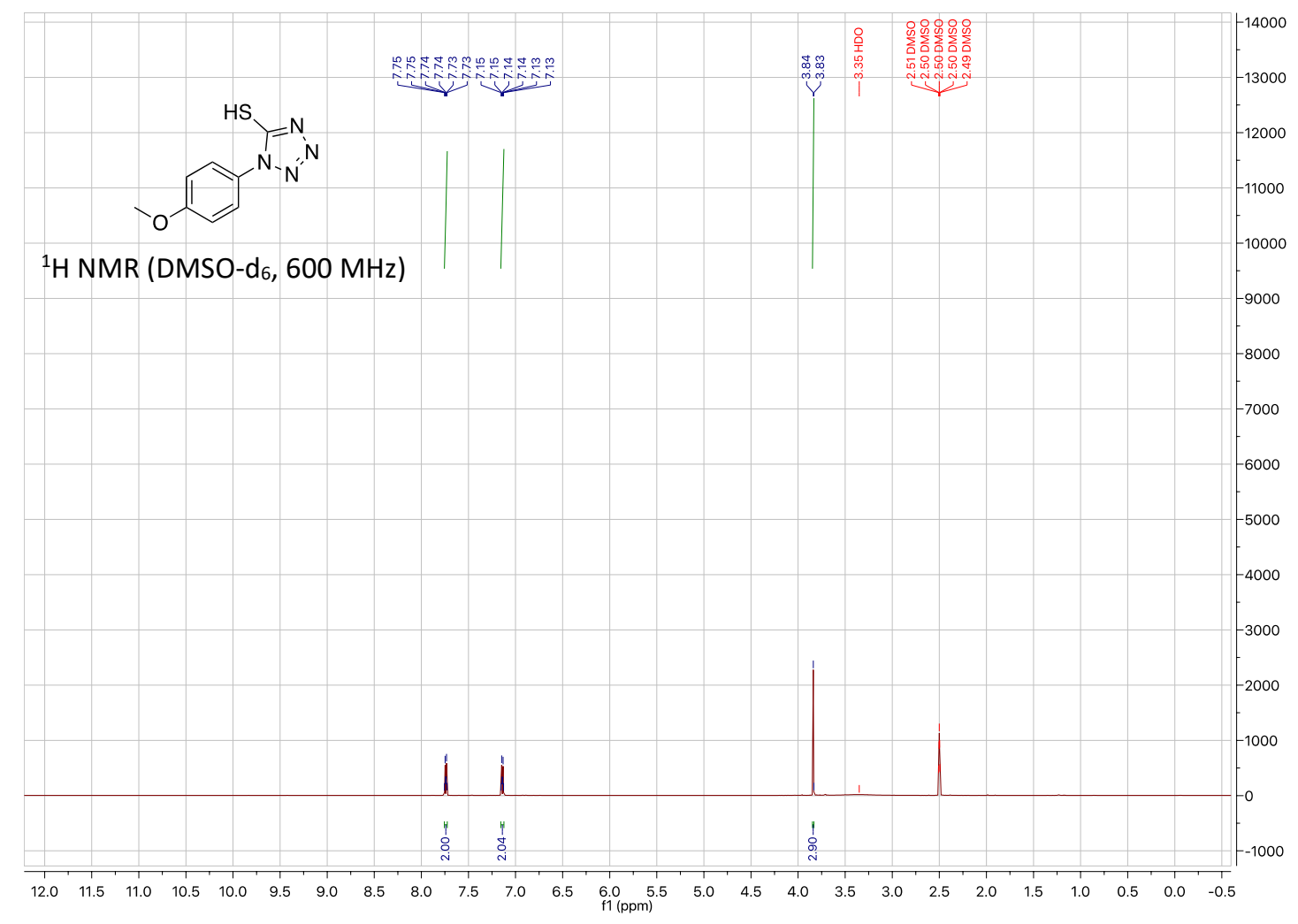

Figure S10. ${ }^{1} \mathrm{H}$ NMR of 1-(4-methoxyphenyl)-1H-tetrazole-5-thiol e (DMSO-d $\mathrm{d}_{6}$ )

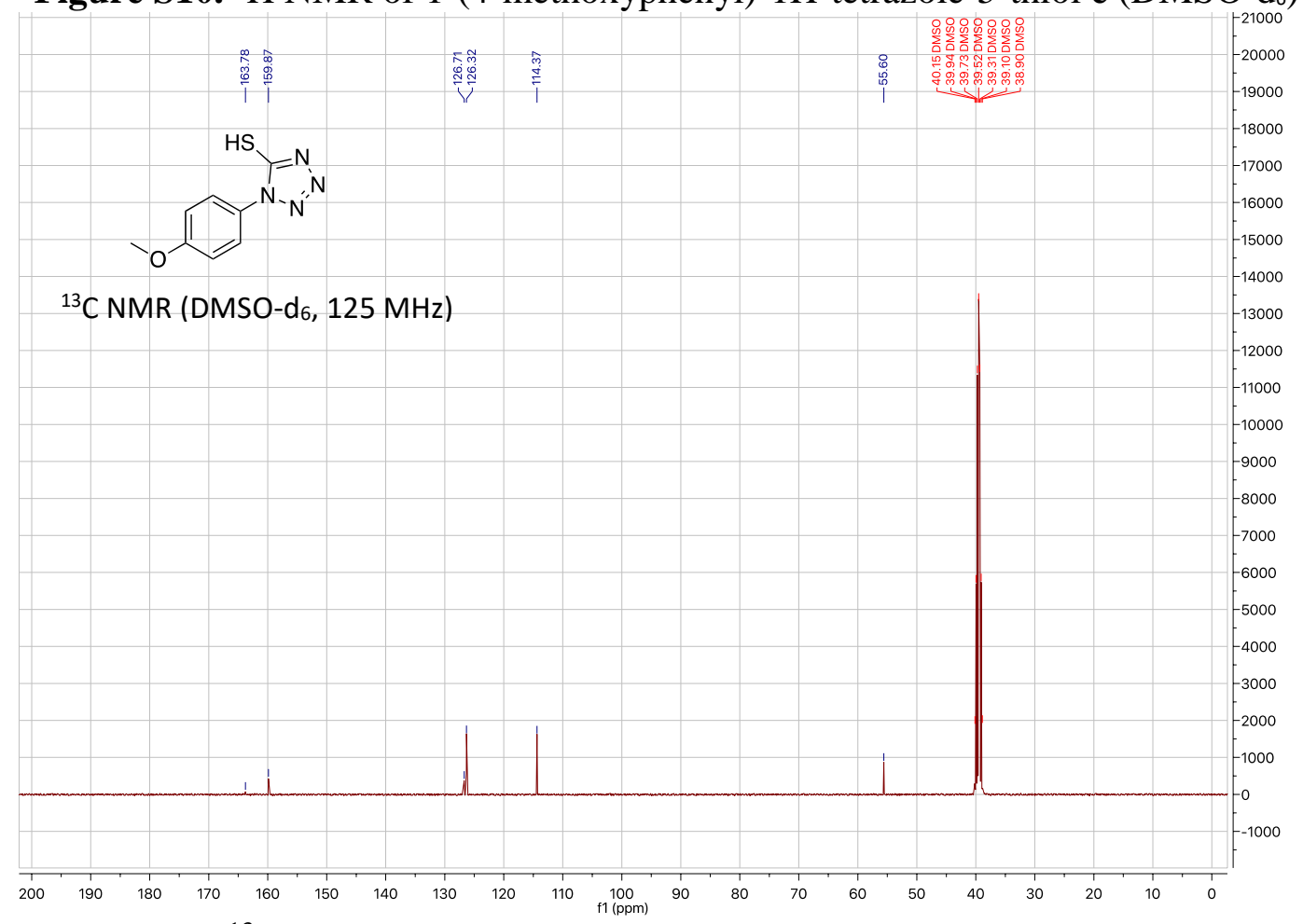

Figure S11. ${ }^{13} \mathrm{C}$ NMR of 1-(4-methoxyphenyl)-1H-tetrazole-5-thiol e (DMSO-d $\left.\mathrm{d}_{6}\right)$ 


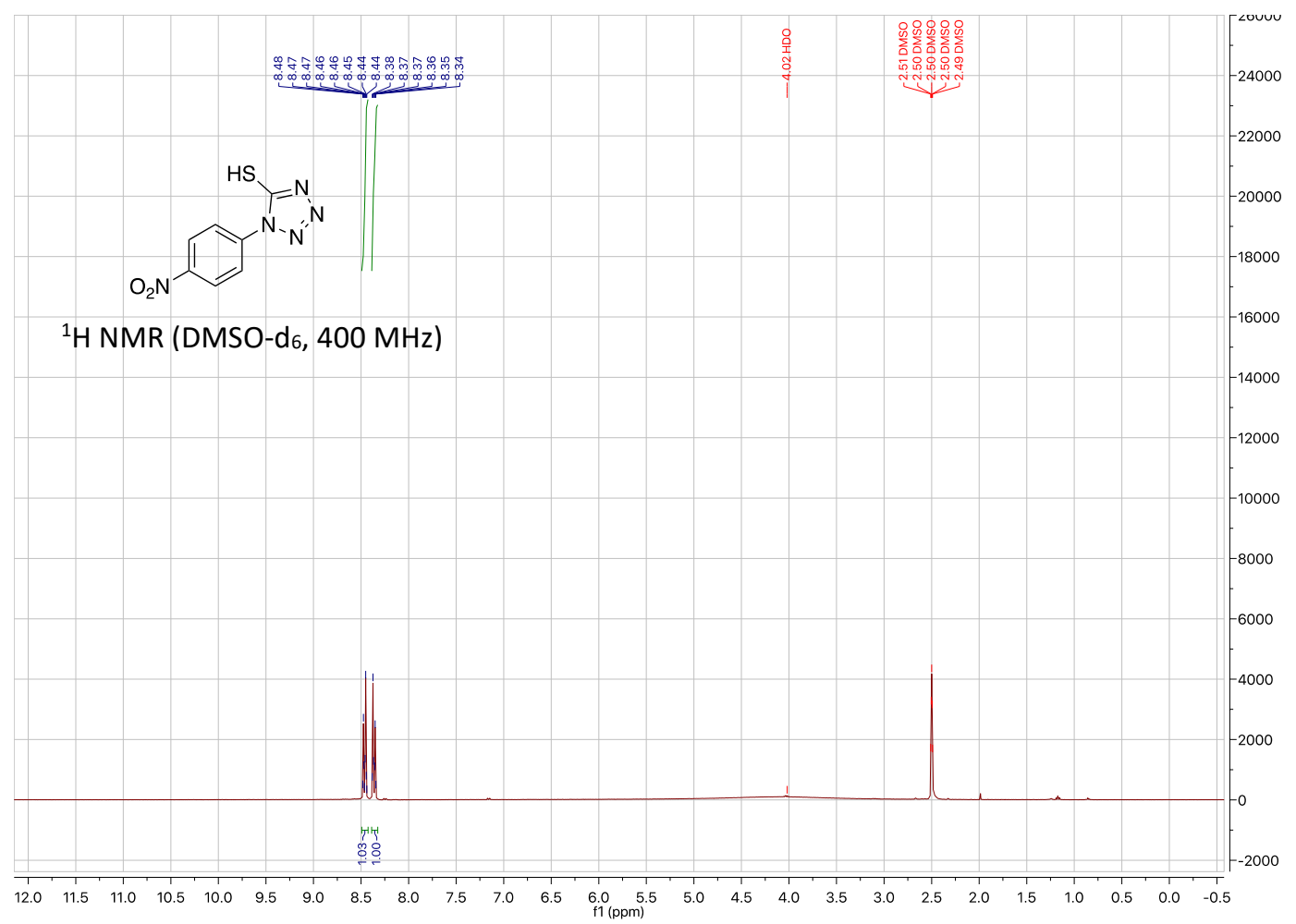

Figure S12. ${ }^{1} \mathrm{H}$ NMR of 1-(4-nitrophenyl)-1H-tetrazole-5-thiol $\mathbf{f}\left(\mathrm{DMSO}^{-} \mathrm{d}_{6}\right)$

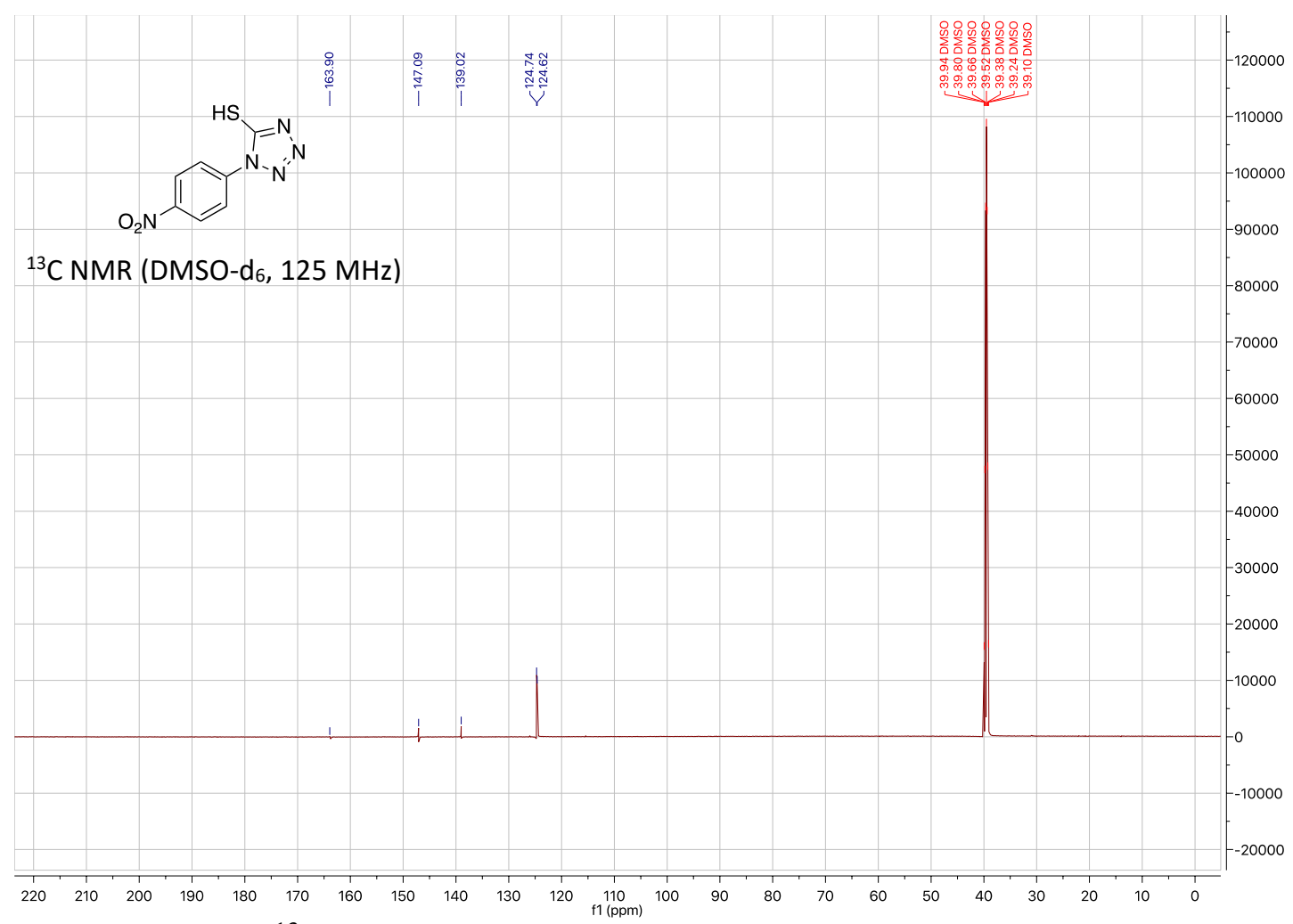

Figure S13. ${ }^{13} \mathrm{C}$ NMR of 1-(4-nitrophenyl)-1H-tetrazole-5-thiol $\mathbf{f}\left(\mathrm{DMSO}-\mathrm{d}_{6}\right)$ 


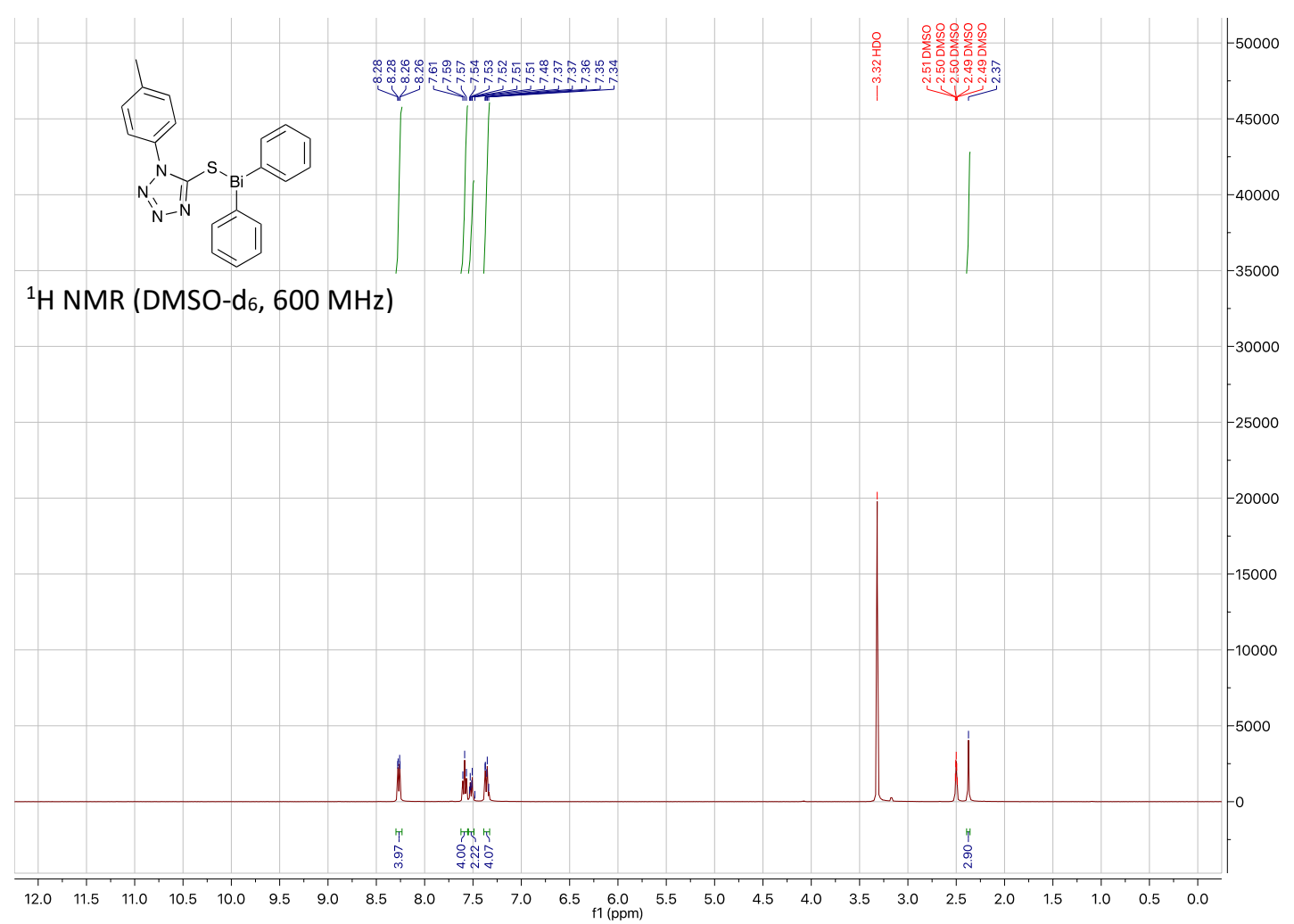

Figure S14. ${ }^{1} \mathrm{H}$ NMR of $\left[\mathrm{Bi}(\mathrm{Ph})_{2}\left(\mathrm{C}_{8} \mathrm{H}_{7} \mathrm{~N}_{4} \mathrm{~S}\right)\right]$ 1a $\left(\mathrm{DMSO}-\mathrm{d}_{6}\right)$

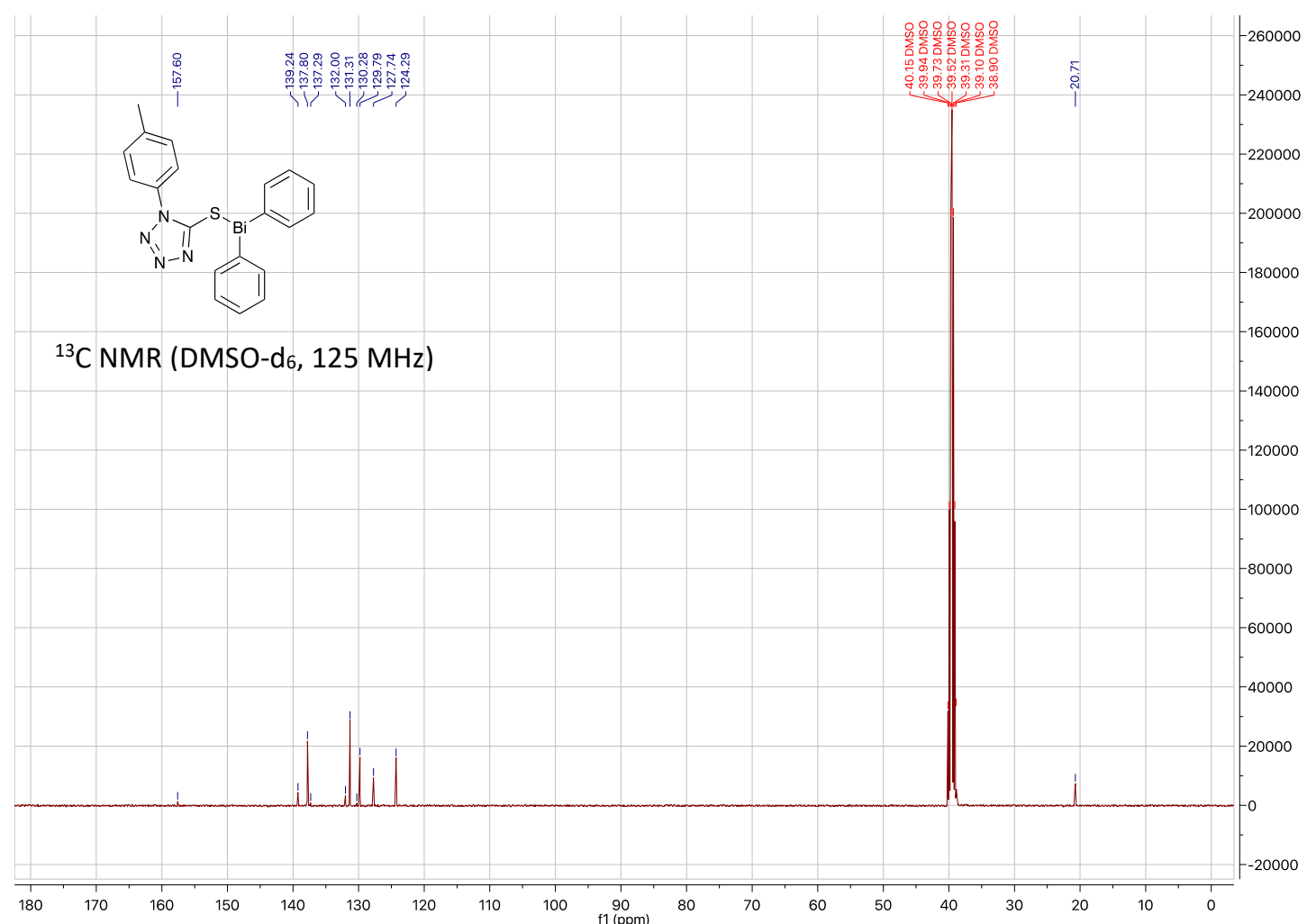

Figure S15. ${ }^{13} \mathrm{C}$ NMR of $\left[\mathrm{Bi}(\mathrm{Ph})_{2}\left(\mathrm{C}_{8} \mathrm{H}_{7} \mathrm{~N}_{4} \mathrm{~S}\right)\right]$ 1a $\left(\mathrm{DMSO}-\mathrm{d}_{6}\right)$ 


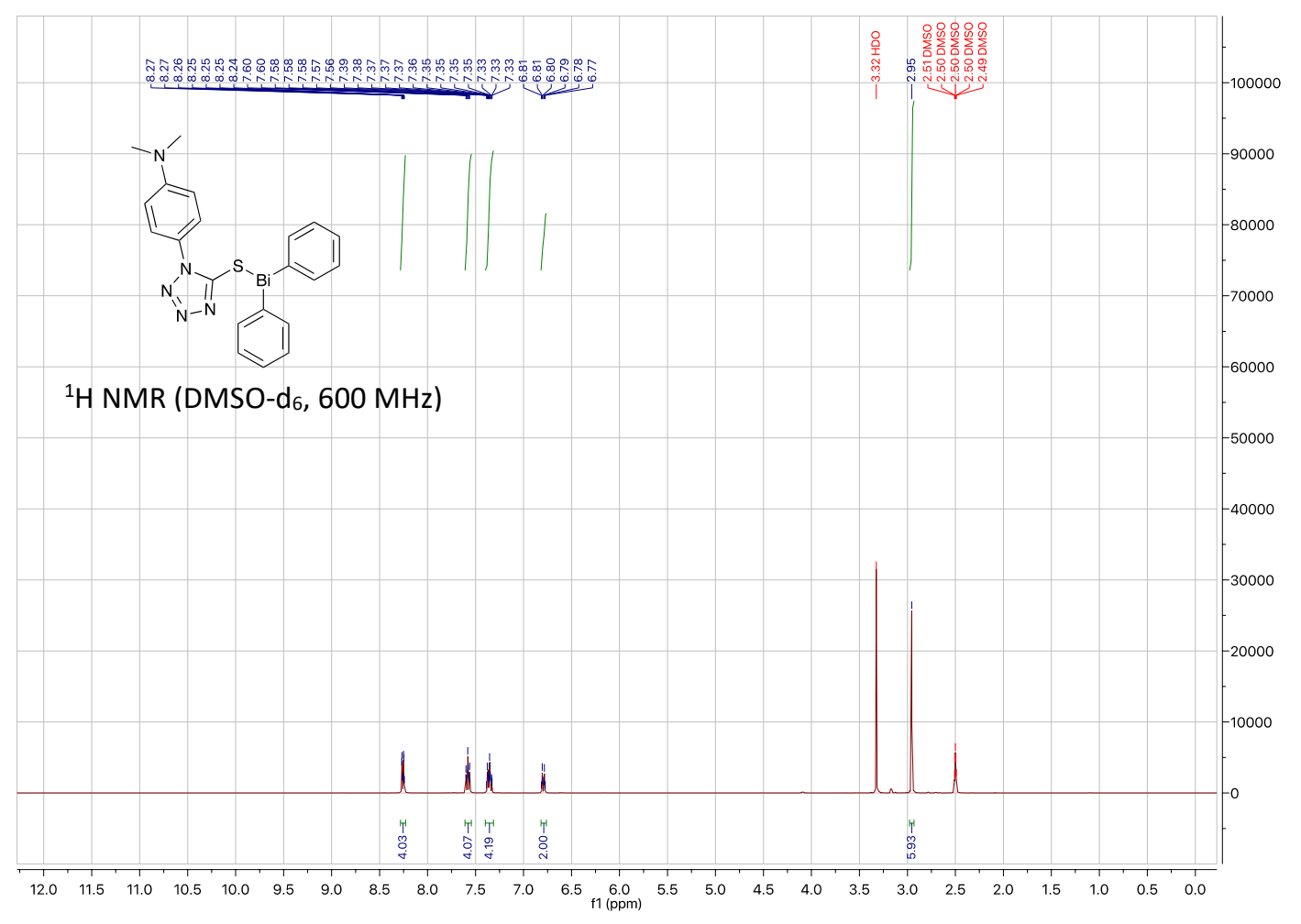

Figure S16. ${ }^{1} \mathrm{H} \mathrm{NMR}$ of $\left[\mathrm{Bi}(\mathrm{Ph})_{2}\left(\mathrm{C}_{9} \mathrm{H}_{10} \mathrm{~N}_{5} \mathrm{~S}\right)\right] \mathbf{1 b}\left(\mathrm{DMSO}-\mathrm{d}_{6}\right)$

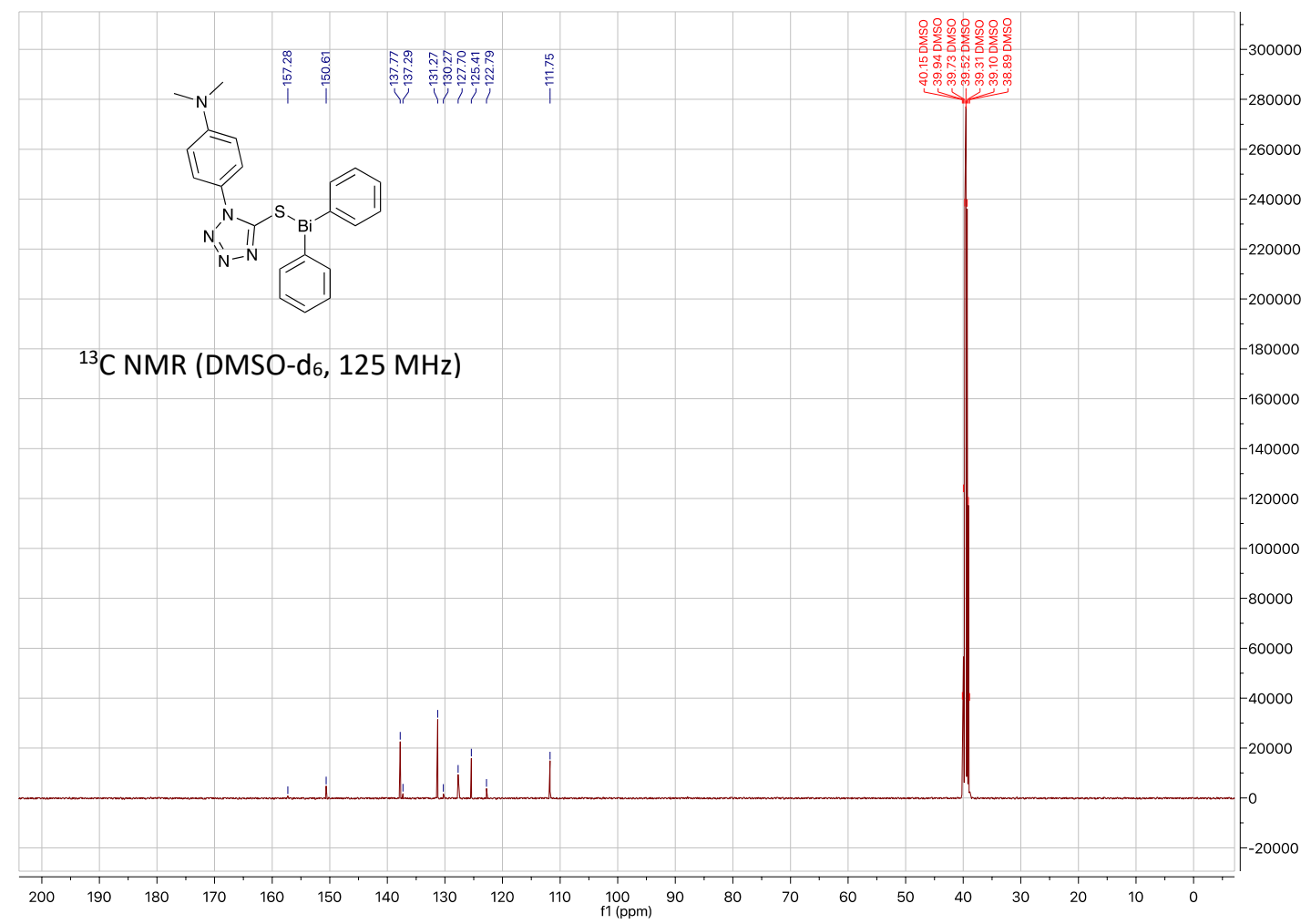

Figure S17. ${ }^{13} \mathrm{C}$ NMR of $\left[\mathrm{Bi}(\mathrm{Ph})_{2}\left(\mathrm{C}_{9} \mathrm{H}_{10} \mathrm{~N}_{5} \mathrm{~S}\right)\right]$ 1b (DMSO-d $\left.\mathrm{d}_{6}\right)$ 


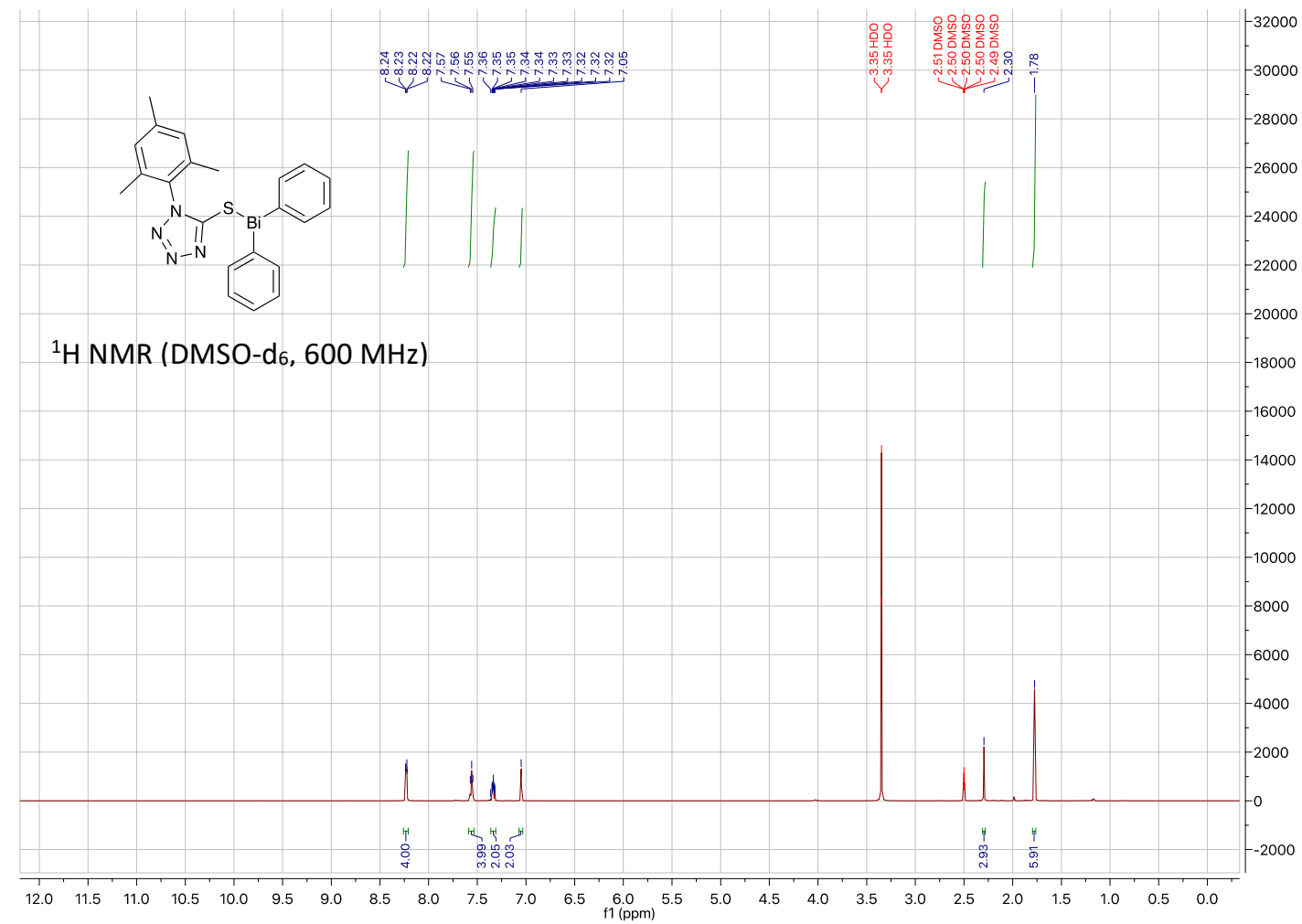

Figure S18. ${ }^{1} \mathrm{H}$ NMR of $\left[\mathrm{Bi}(\mathrm{Ph})_{2}\left(\mathrm{C}_{10} \mathrm{H}_{11} \mathrm{~N}_{4} \mathrm{~S}\right)\right]$ 1c $\left(\mathrm{DMSO}-\mathrm{d}_{6}\right)$

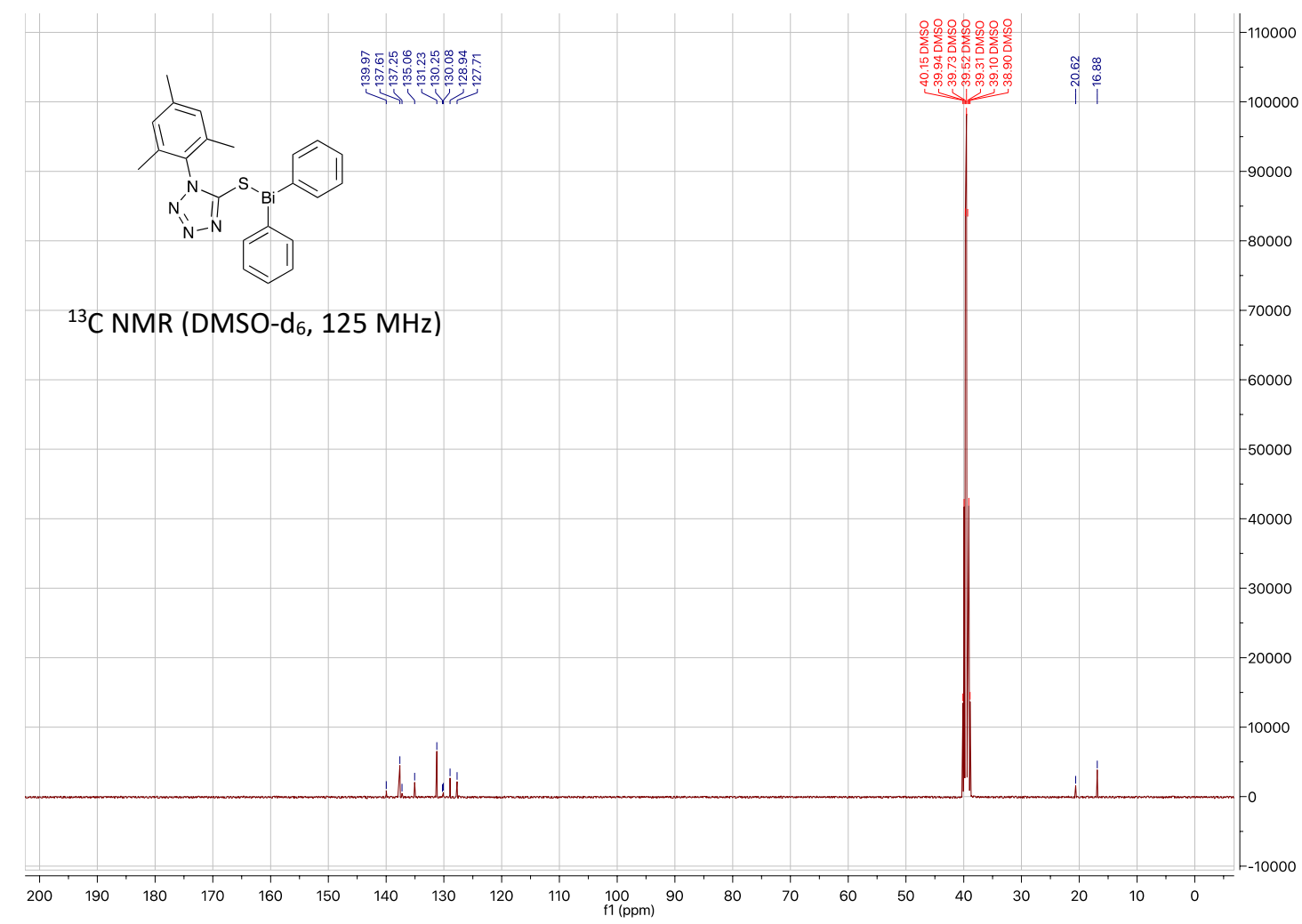

Figure S19. ${ }^{13} \mathrm{C}$ NMR of $\left[\mathrm{Bi}(\mathrm{Ph})_{2}\left(\mathrm{C}_{10} \mathrm{H}_{11} \mathrm{~N}_{4} \mathrm{~S}\right)\right] 1 \mathrm{c}\left(\mathrm{DMSO}-\mathrm{d}_{6}\right)$ 


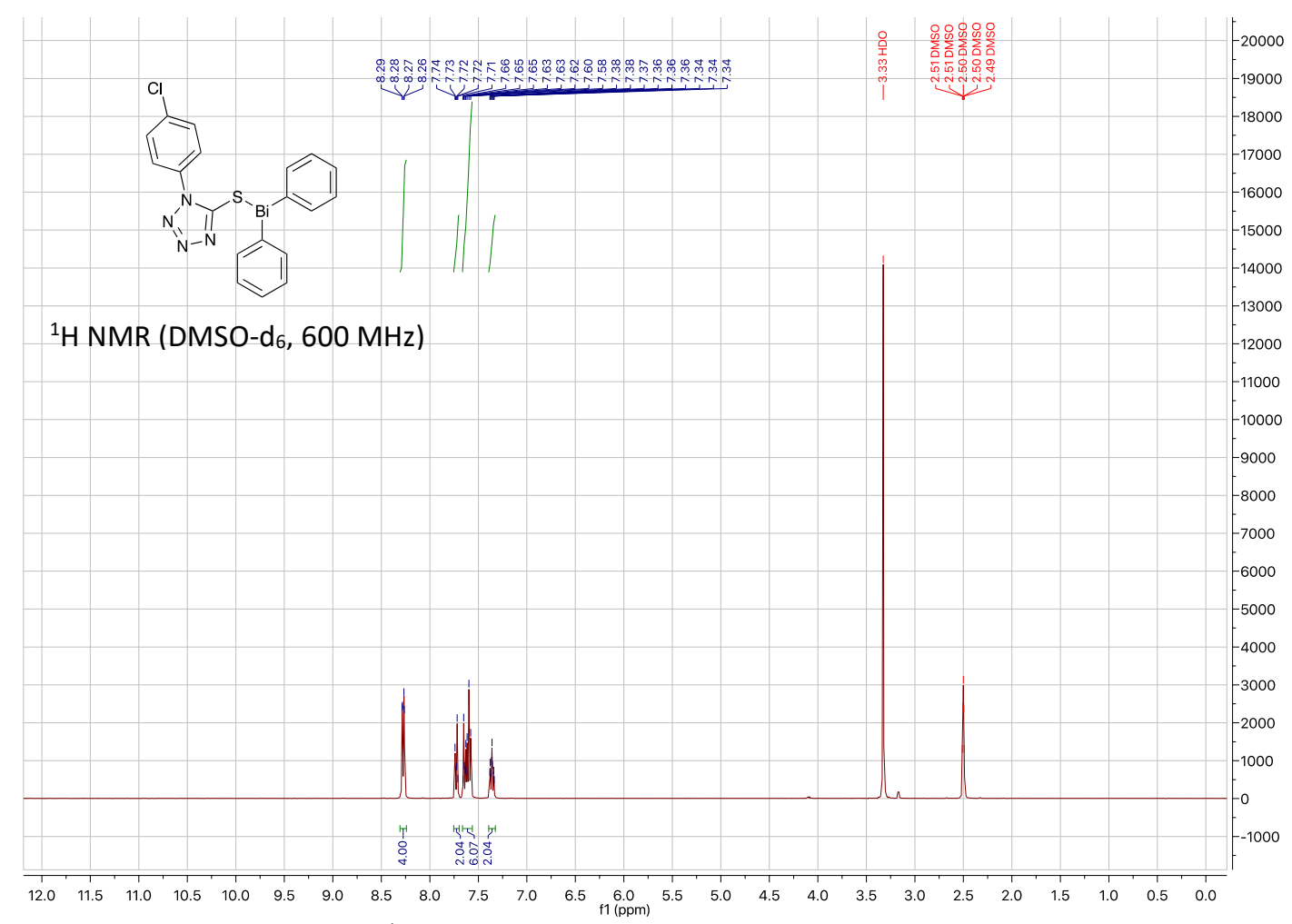

Figure S20. ${ }^{1} \mathrm{H}$ NMR of $\left[\mathrm{Bi}(\mathrm{Ph})_{2}\left(\mathrm{C}_{7} \mathrm{H}_{4} \mathrm{~N}_{4} \mathrm{ClS}\right)\right]$ 1d $\left(\mathrm{DMSO}-\mathrm{d}_{6}\right)$

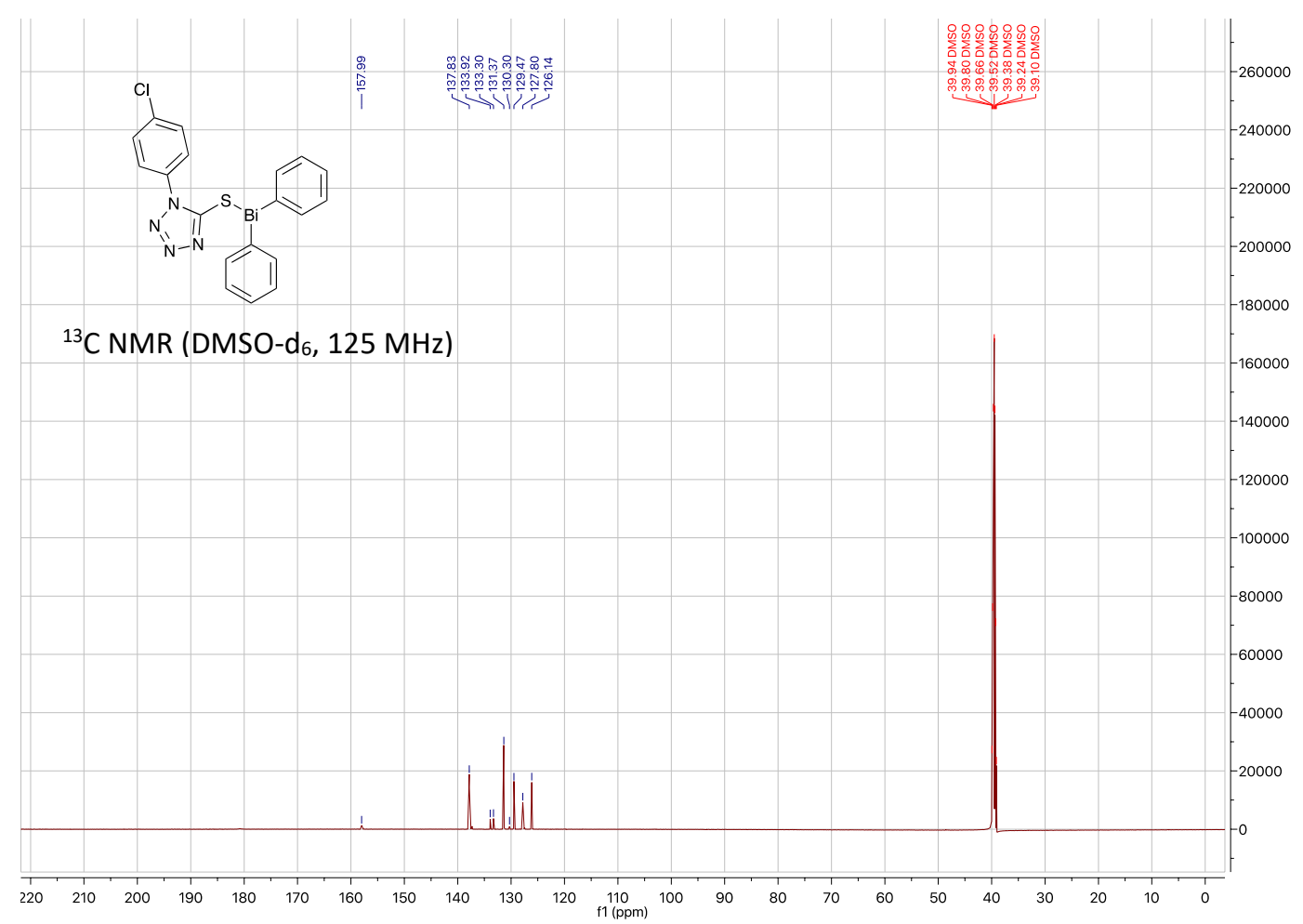

Figure S21. ${ }^{13} \mathrm{C}$ NMR of $\left[\mathrm{Bi}(\mathrm{Ph})_{2}\left(\mathrm{C}_{7} \mathrm{H}_{4} \mathrm{~N}_{4} \mathrm{ClS}\right)\right]$ 1d $\left(\mathrm{DMSO}-\mathrm{d}_{6}\right)$ 


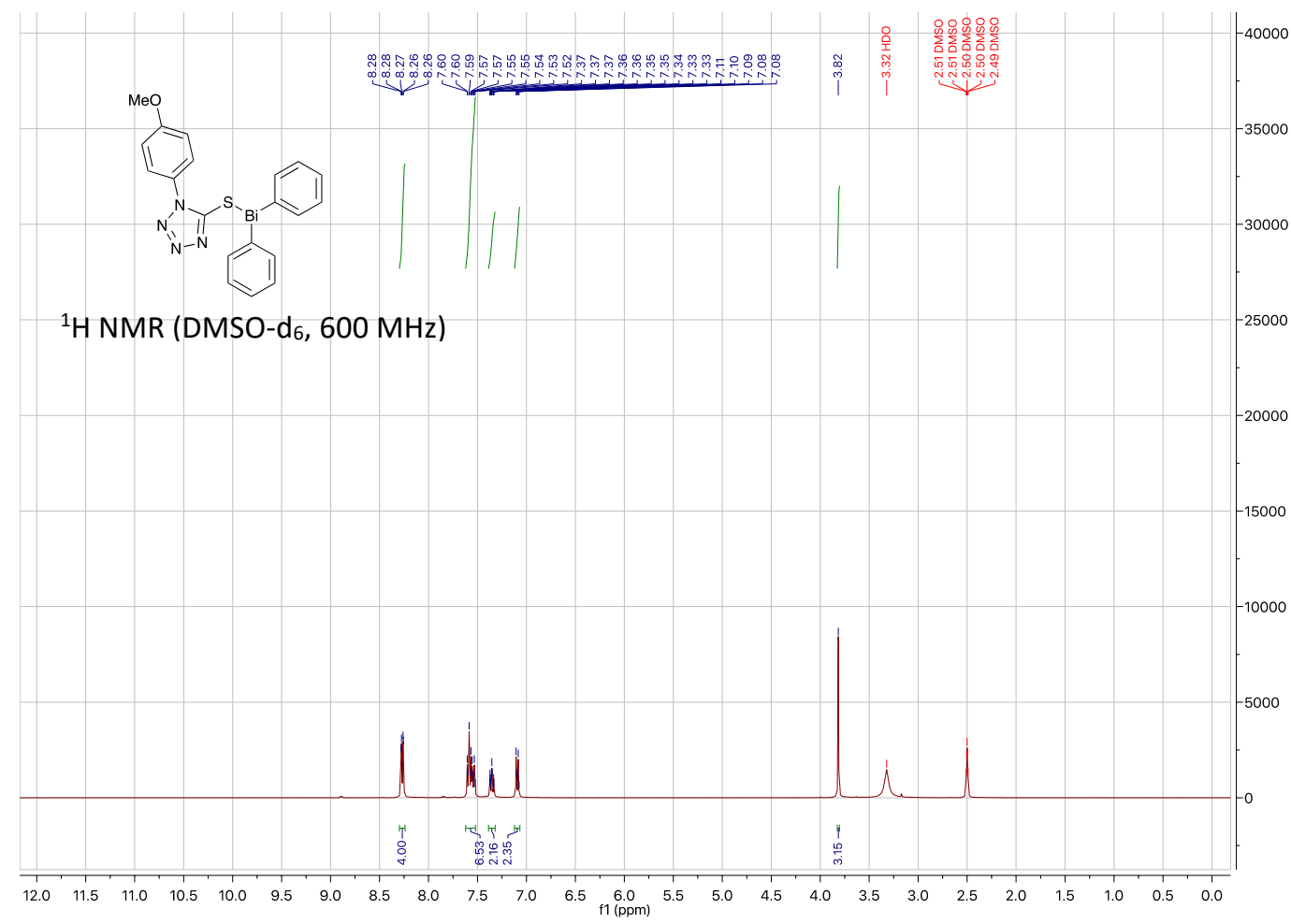

Figure S22. ${ }^{1} \mathrm{H}$ NMR of $\left[\mathrm{Bi}(\mathrm{Ph})_{2}\left(\mathrm{C}_{8} \mathrm{H}_{7} \mathrm{~N}_{4} \mathrm{OS}\right)\right]$ 1e $\left(\mathrm{DMSO}-\mathrm{d}_{6}\right)$

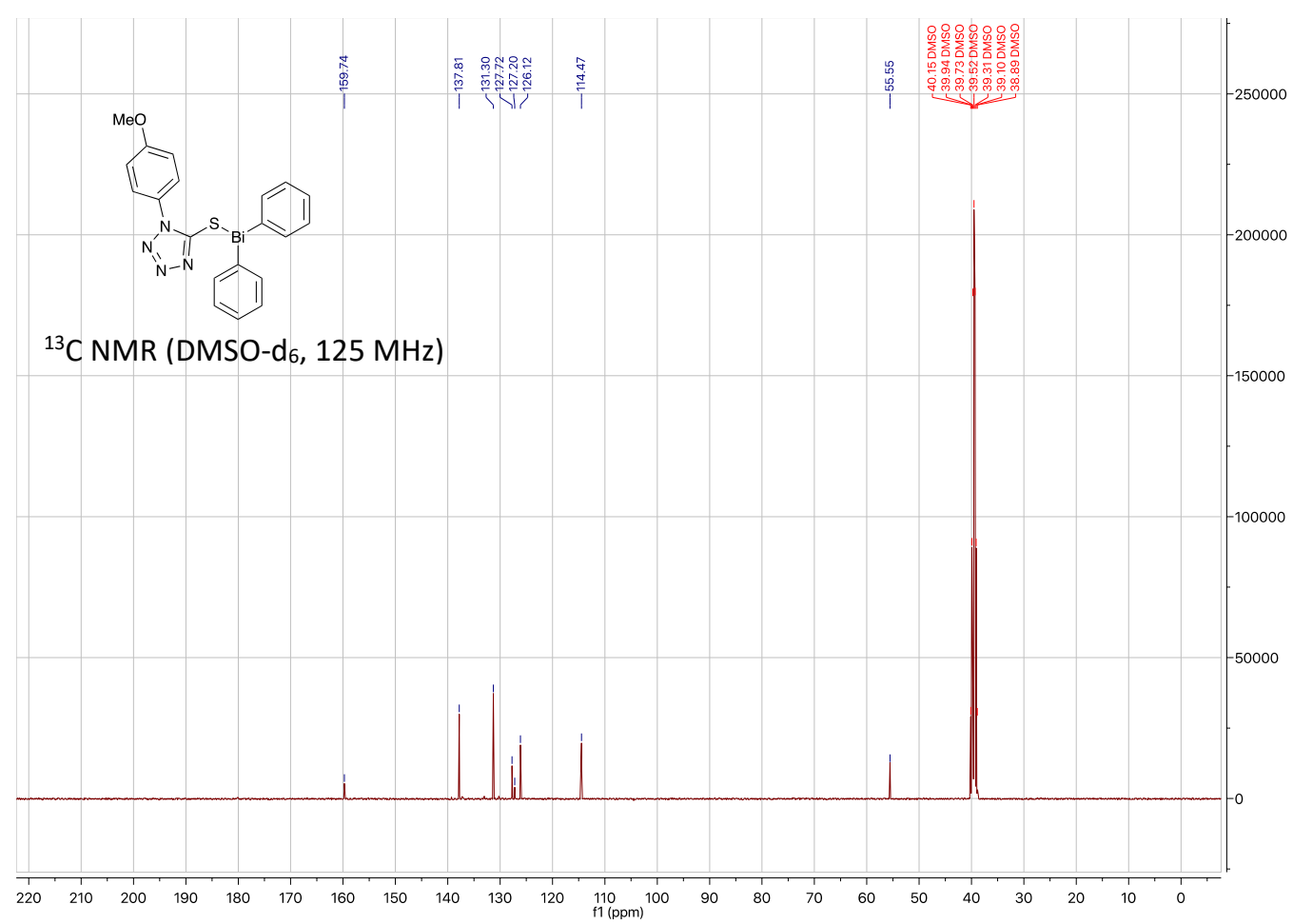

Figure S23. ${ }^{13} \mathrm{C} \mathrm{NMR}$ of $\left[\mathrm{Bi}(\mathrm{Ph})_{2}\left(\mathrm{C}_{8} \mathrm{H}_{7} \mathrm{~N}_{4} \mathrm{OS}\right)\right] \mathbf{1 e}\left(\mathrm{DMSO}-\mathrm{d}_{6}\right)$ 


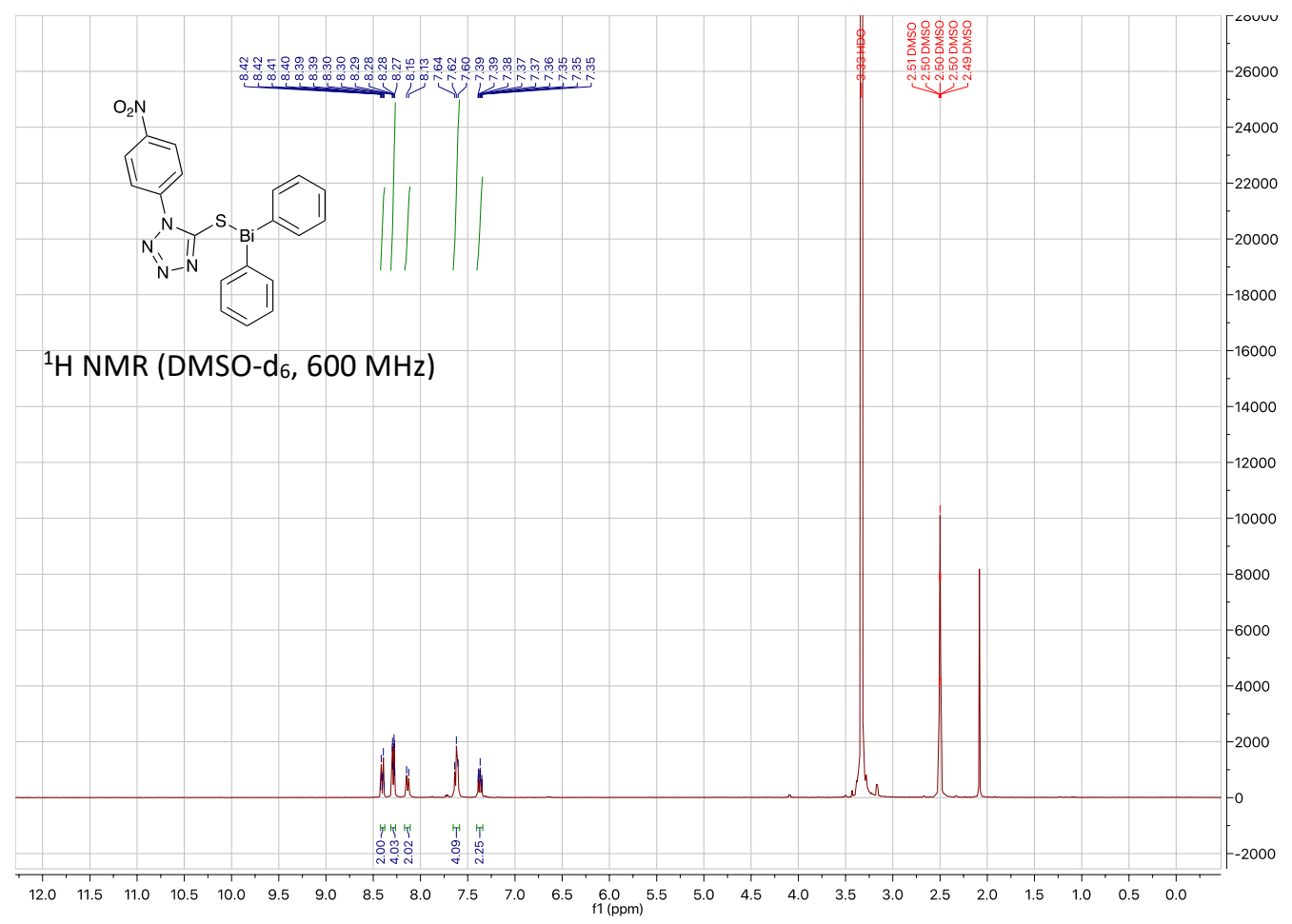

Figure S24. ${ }^{1} \mathrm{H}$ NMR of $\left[\mathrm{Bi}(\mathrm{Ph})_{2}\left(\mathrm{C}_{7} \mathrm{H}_{4} \mathrm{~N}_{5} \mathrm{O}_{2} \mathrm{~S}\right)\right]$ 1f $\left(\mathrm{DMSO}-\mathrm{d}_{6}\right)$

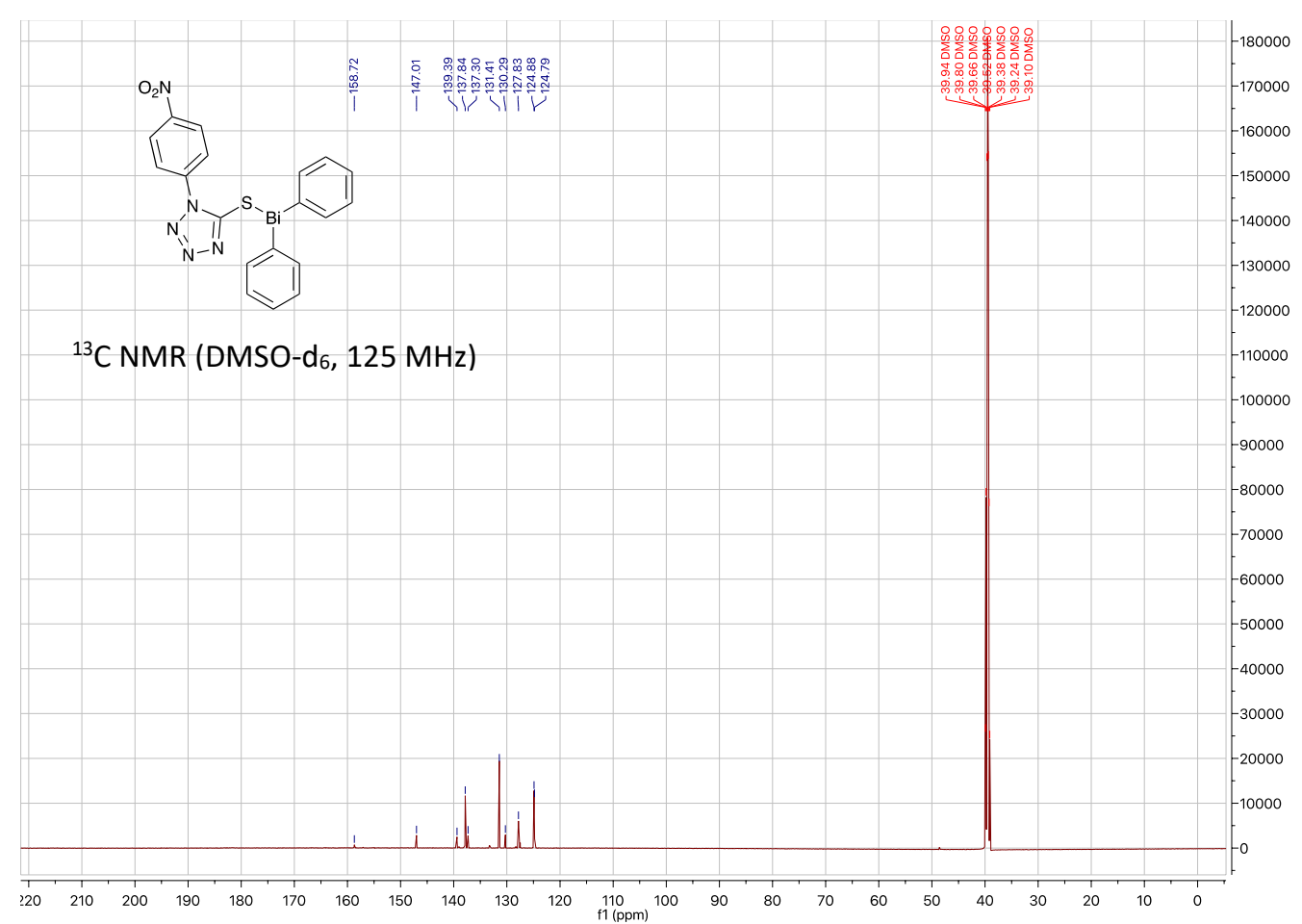

Figure S25. ${ }^{13} \mathrm{C}$ NMR of $\left[\mathrm{Bi}(\mathrm{Ph})_{2}\left(\mathrm{C}_{7} \mathrm{H}_{4} \mathrm{~N}_{5} \mathrm{O}_{2} \mathrm{~S}\right)\right] \mathbf{1 f}\left(\mathrm{DMSO}-\mathrm{d}_{6}\right)$ 


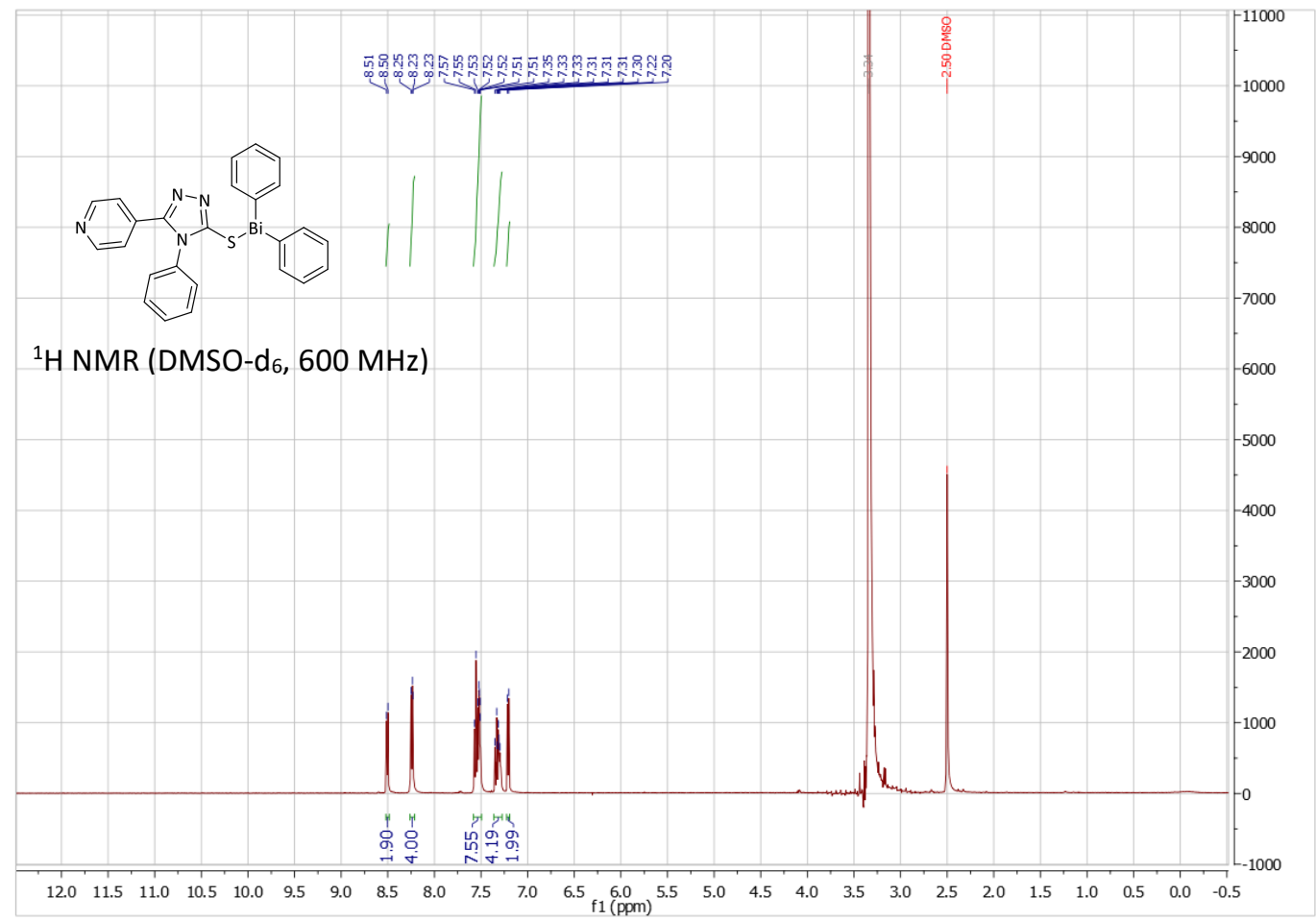

Figure S26. ${ }^{1} \mathrm{H} \mathrm{NMR}$ of $\left[\mathrm{Bi}(\mathrm{Ph})_{2}\left(\mathrm{C}_{13} \mathrm{H}_{9} \mathrm{~N}_{4} \mathrm{~S}\right)\right] 1 \mathrm{1g}\left(\mathrm{DMSO}-\mathrm{d}_{6}\right)$

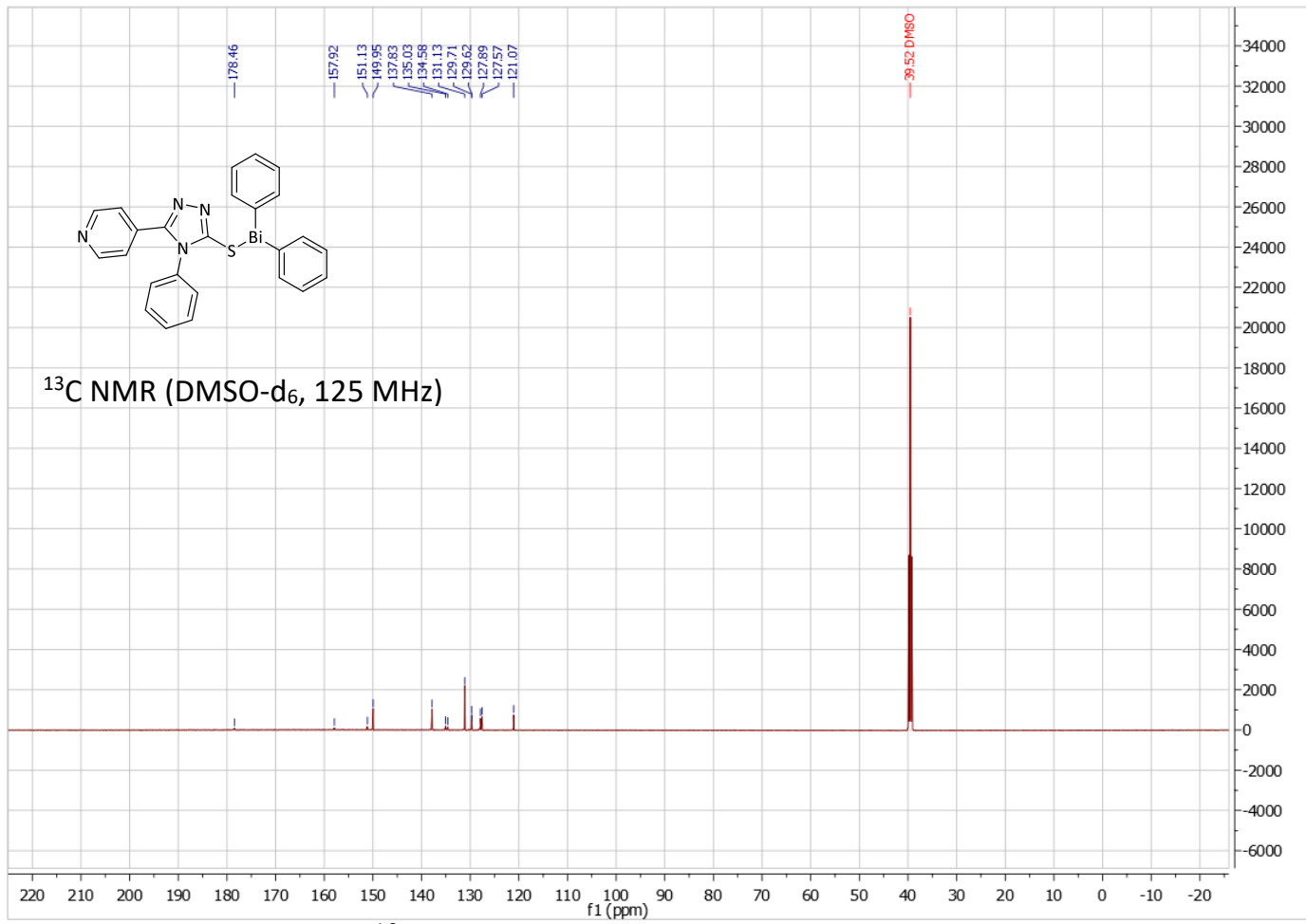

Figure S27. ${ }^{13} \mathrm{C}$ NMR of $\left[\mathrm{Bi}(\mathrm{Ph})_{2}\left(\mathrm{C}_{13} \mathrm{H}_{9} \mathrm{~N}_{4} \mathrm{~S}\right)\right] \mathbf{1 g}\left(\mathrm{DMSO}-\mathrm{d}_{6}\right)$ 


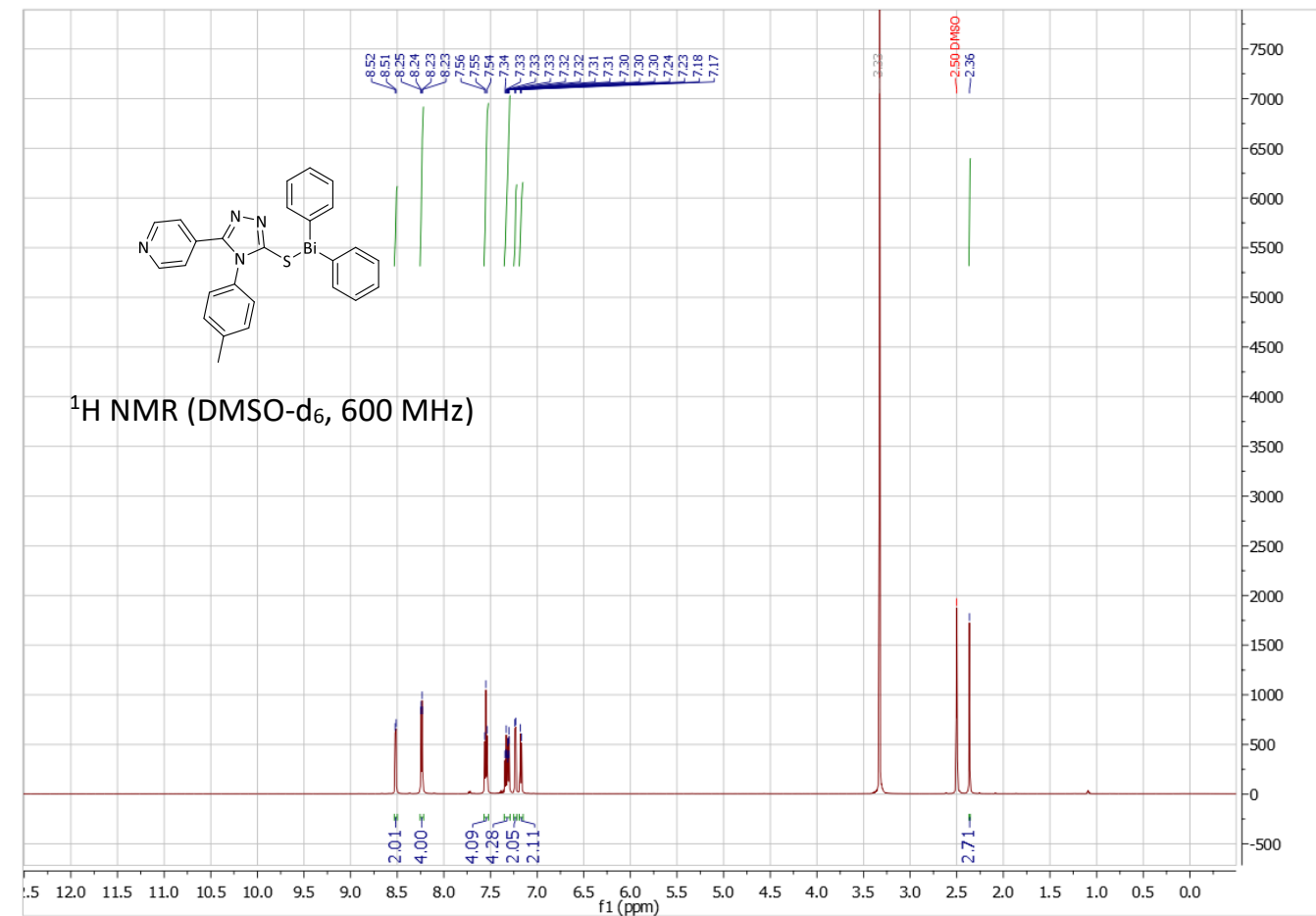

Figure S28. ${ }^{1} \mathrm{H}$ NMR of $\left[\mathrm{Bi}(\mathrm{Ph})_{2}\left(\mathrm{C}_{14} \mathrm{H}_{11} \mathrm{~N}_{4} \mathrm{~S}\right)\right]$ 1h $\left(\mathrm{DMSO}-\mathrm{d}_{6}\right)$

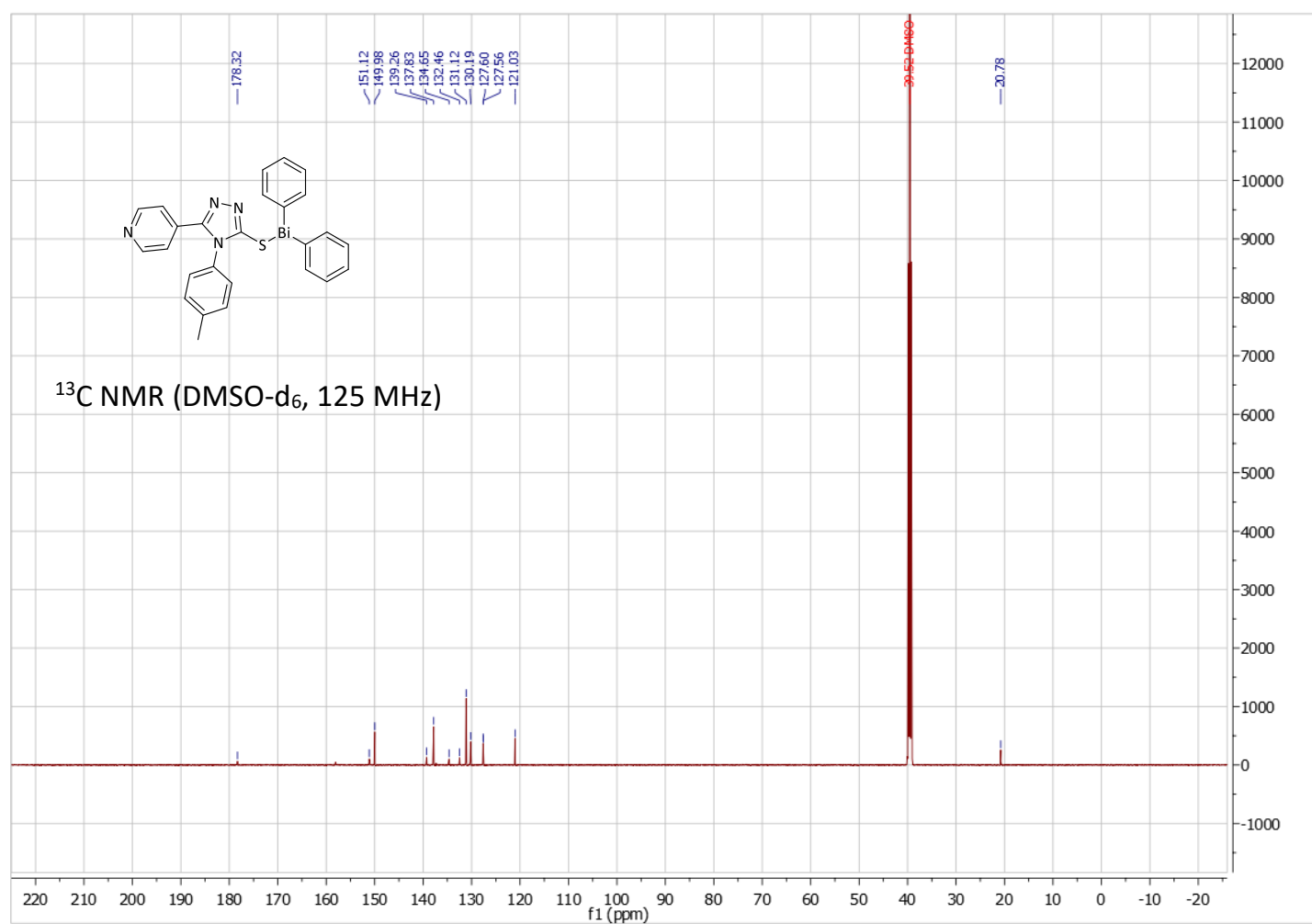

Figure S29. ${ }^{13} \mathrm{C}$ NMR of $\left[\mathrm{Bi}(\mathrm{Ph})_{2}\left(\mathrm{C}_{14} \mathrm{H}_{11} \mathrm{~N}_{4} \mathrm{~S}\right)\right]$ 1h $\left(\mathrm{DMSO}-\mathrm{d}_{6}\right)$ 


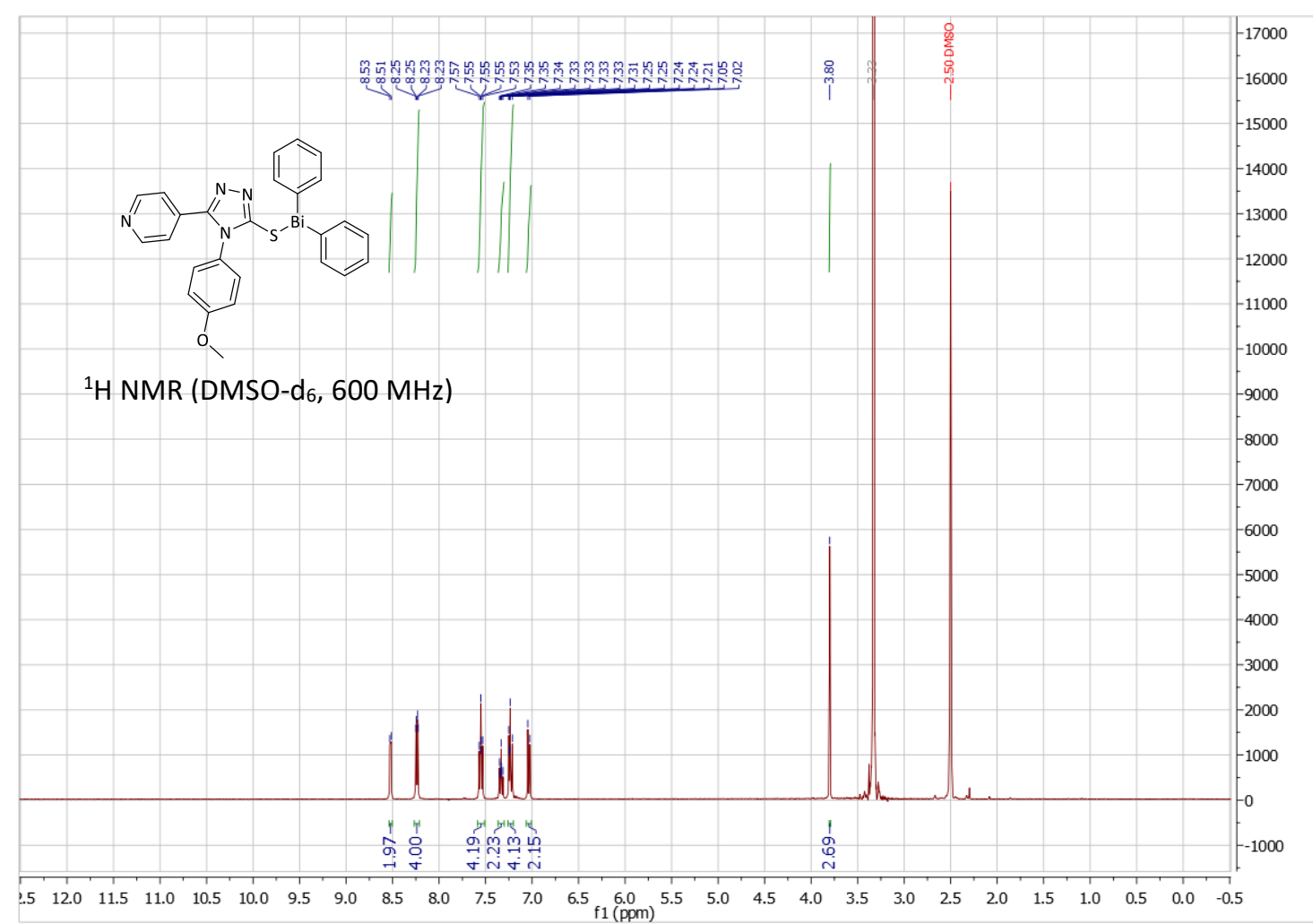

Figure S30. ${ }^{1} \mathrm{H}$ NMR of $\left[\mathrm{Bi}(\mathrm{Ph})_{2}\left(\mathrm{C}_{14} \mathrm{H}_{11} \mathrm{~N}_{4} \mathrm{OS}\right)\right] \mathbf{1} \mathbf{i}\left(\mathrm{DMSO}-\mathrm{d}_{6}\right)$

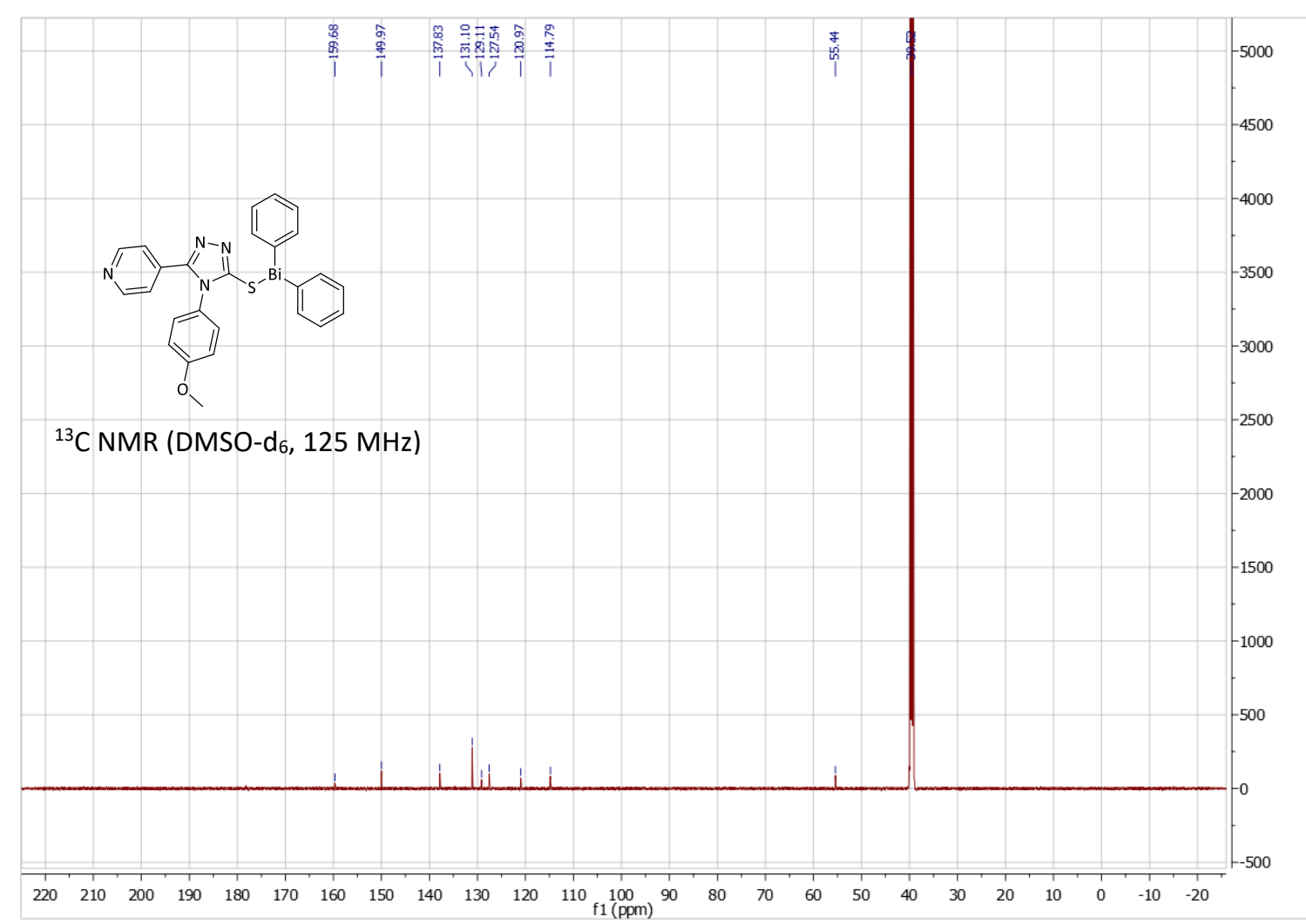

Figure S31. ${ }^{1} \mathrm{H}$ NMR of $\left[\mathrm{Bi}(\mathrm{Ph})_{2}\left(\mathrm{C}_{14} \mathrm{H}_{11} \mathrm{~N}_{4} \mathrm{OS}\right)\right]$ 1i $\left(\mathrm{DMSO}_{-} \mathrm{d}_{6}\right)$ 


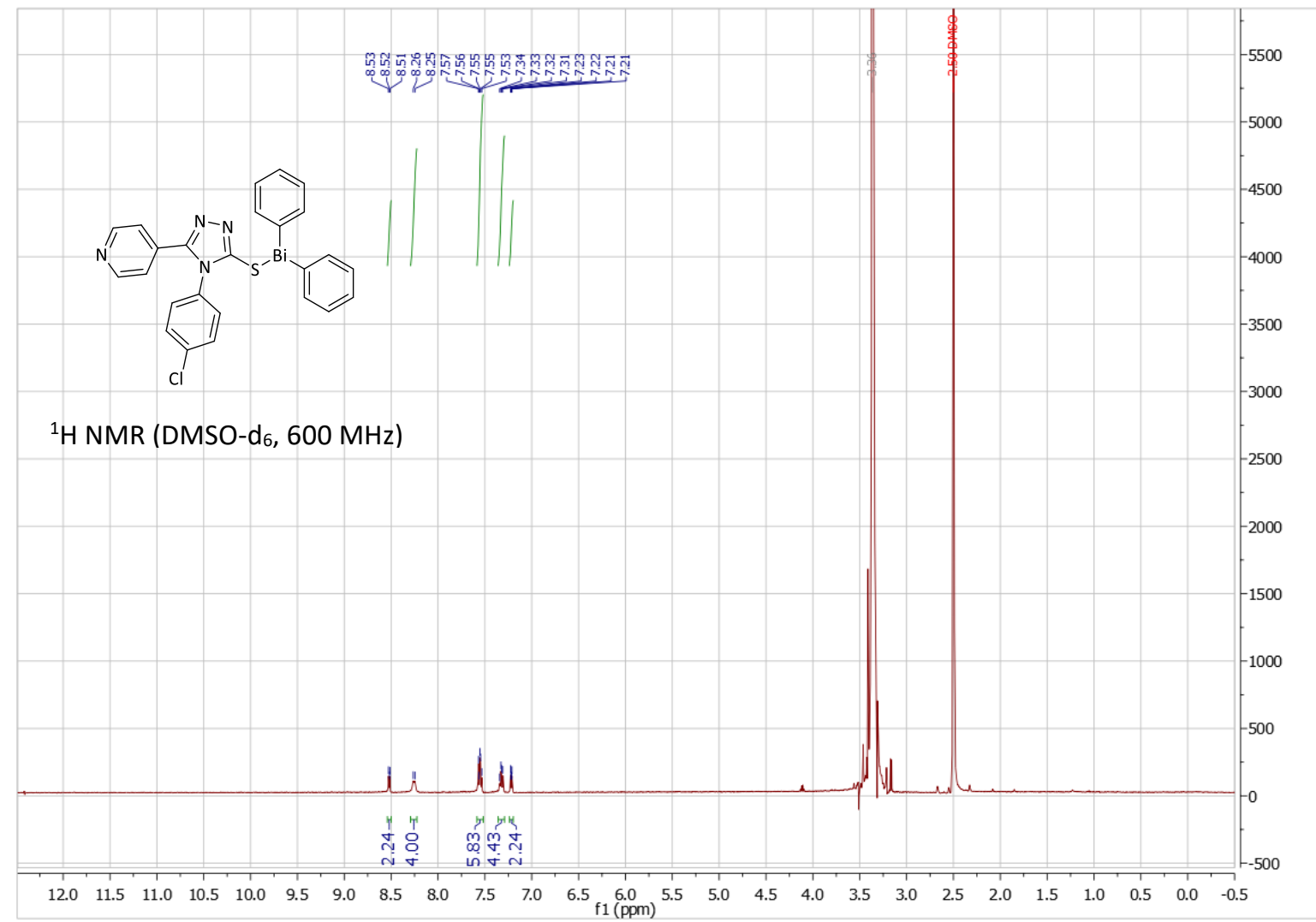

Figure S32. ${ }^{1} \mathrm{H}$ NMR of $\left[\mathrm{Bi}(\mathrm{Ph})_{2}\left(\mathrm{C}_{13} \mathrm{H}_{8} \mathrm{~N}_{4} \mathrm{ClS}\right)\right] \mathbf{1} \mathbf{j}\left(\mathrm{DMSO}-\mathrm{d}_{6}\right)$

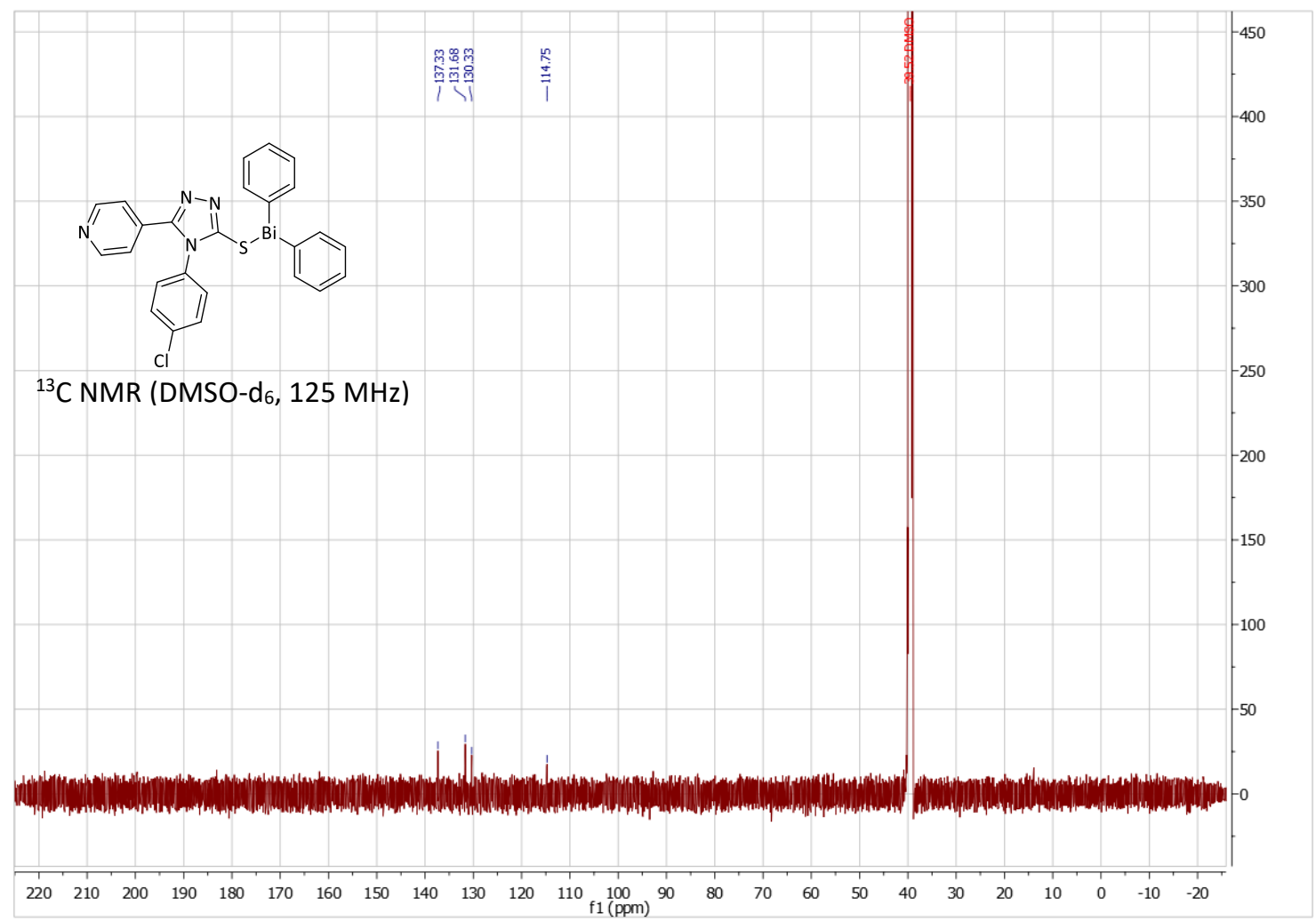

Figure S33. ${ }^{13} \mathrm{C}$ NMR of $\left[\mathrm{Bi}(\mathrm{Ph})_{2}\left(\mathrm{C}_{13} \mathrm{H}_{8} \mathrm{~N}_{4} \mathrm{ClS}\right)\right] \mathbf{1 j}$ (DMSO-d $\left.\mathrm{d}_{6}\right)$ 


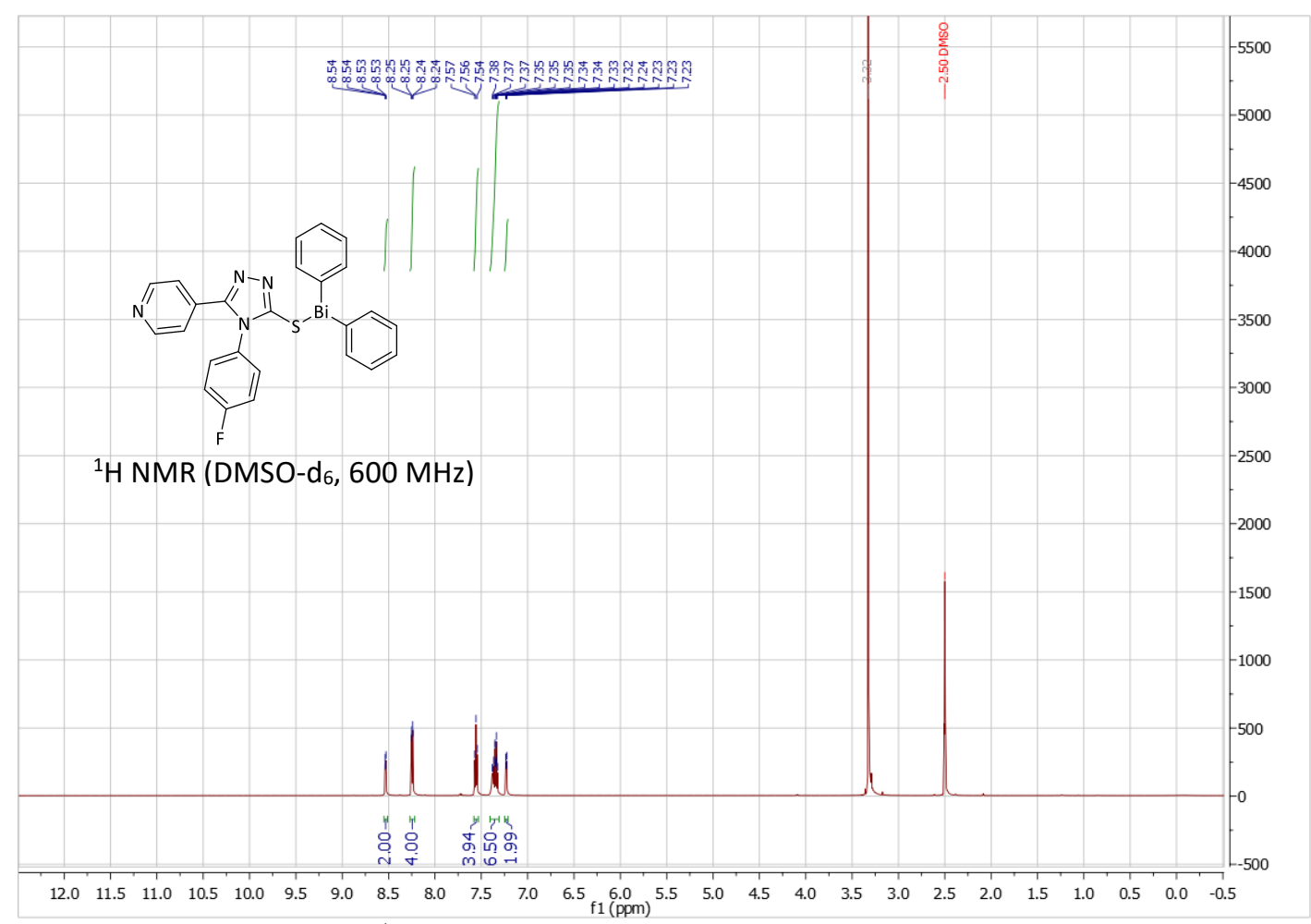

Figure S34. ${ }^{1} \mathrm{H}$ NMR of $\left[\mathrm{Bi}(\mathrm{Ph})_{2}\left(\mathrm{C}_{13} \mathrm{H}_{8} \mathrm{~N}_{4} \mathrm{FS}\right)\right]$ 1k (DMSO- $\left.\mathrm{d}_{6}\right)$

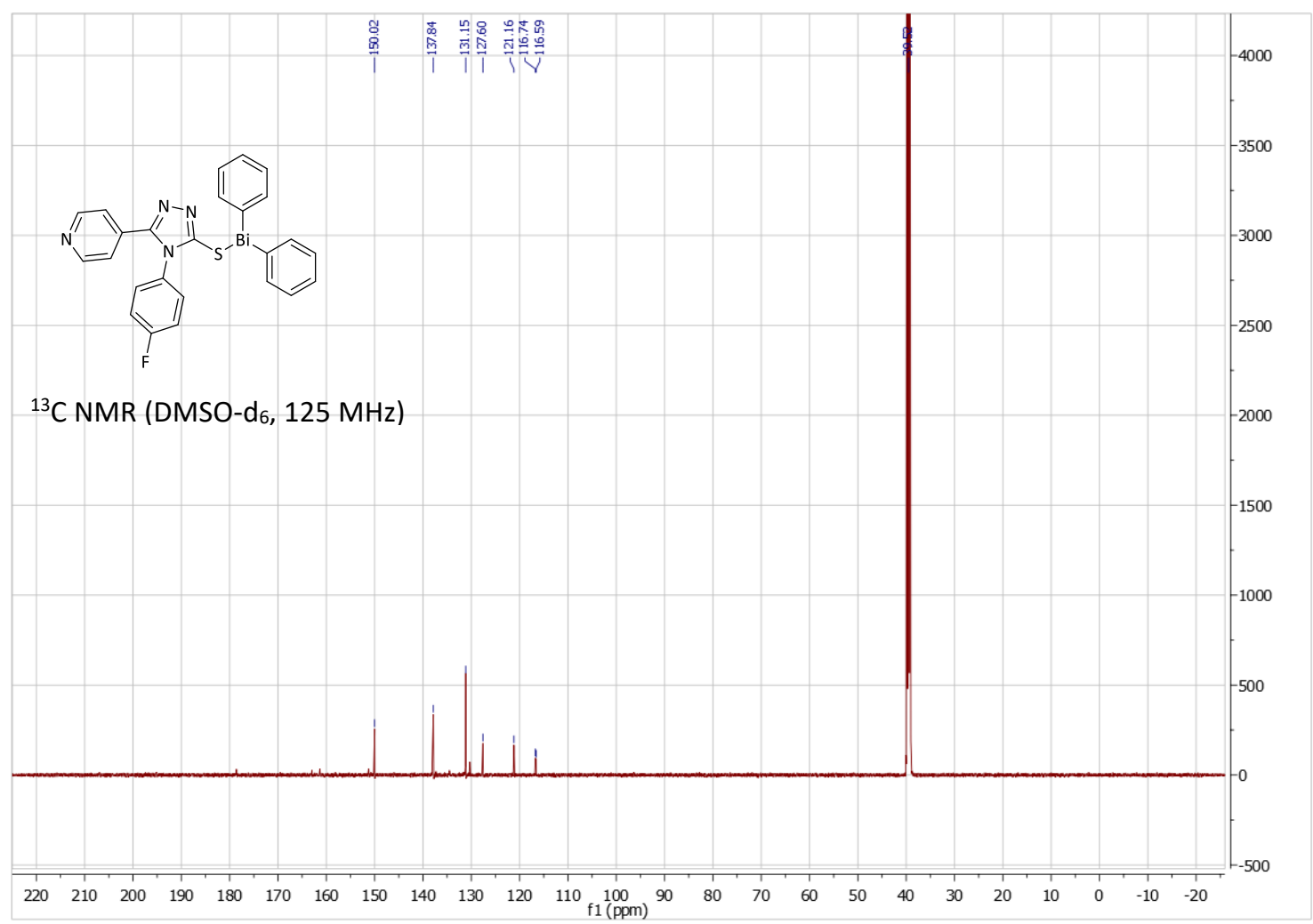

Figure S35. ${ }^{13} \mathrm{C}$ NMR of $\left[\mathrm{Bi}(\mathrm{Ph})_{2}\left(\mathrm{C}_{13} \mathrm{H}_{8} \mathrm{~N}_{4} \mathrm{FS}\right)\right]$ 1k (DMSO- $\left.\mathrm{d}_{6}\right)$ 


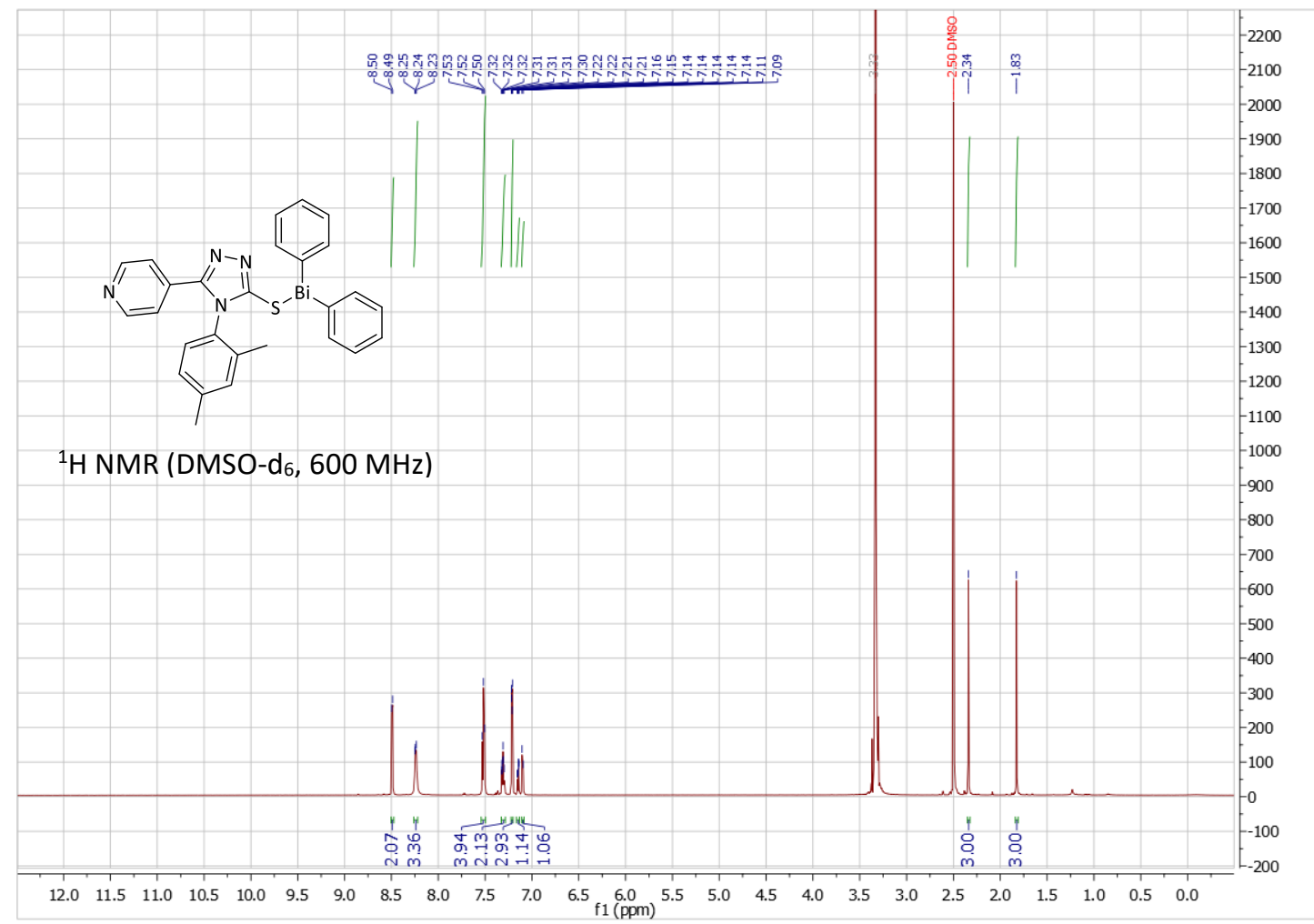

Figure S36. ${ }^{1} \mathrm{H}$ NMR of $\left[\mathrm{Bi}(\mathrm{Ph})_{2}\left(\mathrm{C}_{15} \mathrm{H}_{13} \mathrm{~N}_{4} \mathrm{~S}\right)\right] 1 \mathrm{ll}\left(\mathrm{DMSO}-\mathrm{d}_{6}\right)$

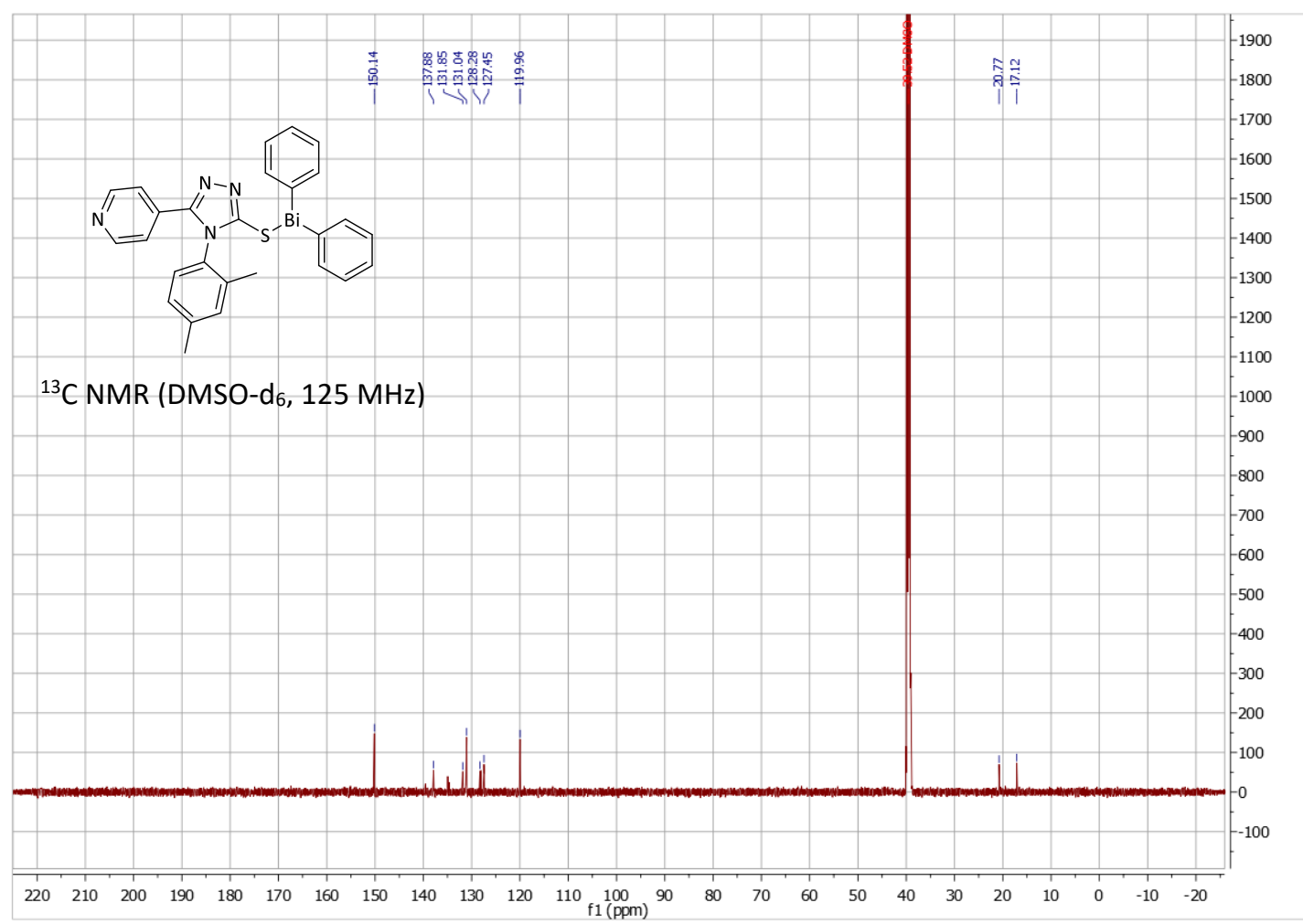

Figure S37. ${ }^{13} \mathrm{C}$ NMR of $\left[\mathrm{Bi}(\mathrm{Ph})_{2}\left(\mathrm{C}_{15} \mathrm{H}_{13} \mathrm{~N}_{4} \mathrm{~S}\right)\right] 11\left(\mathrm{DMSO}-\mathrm{d}_{6}\right)$ 


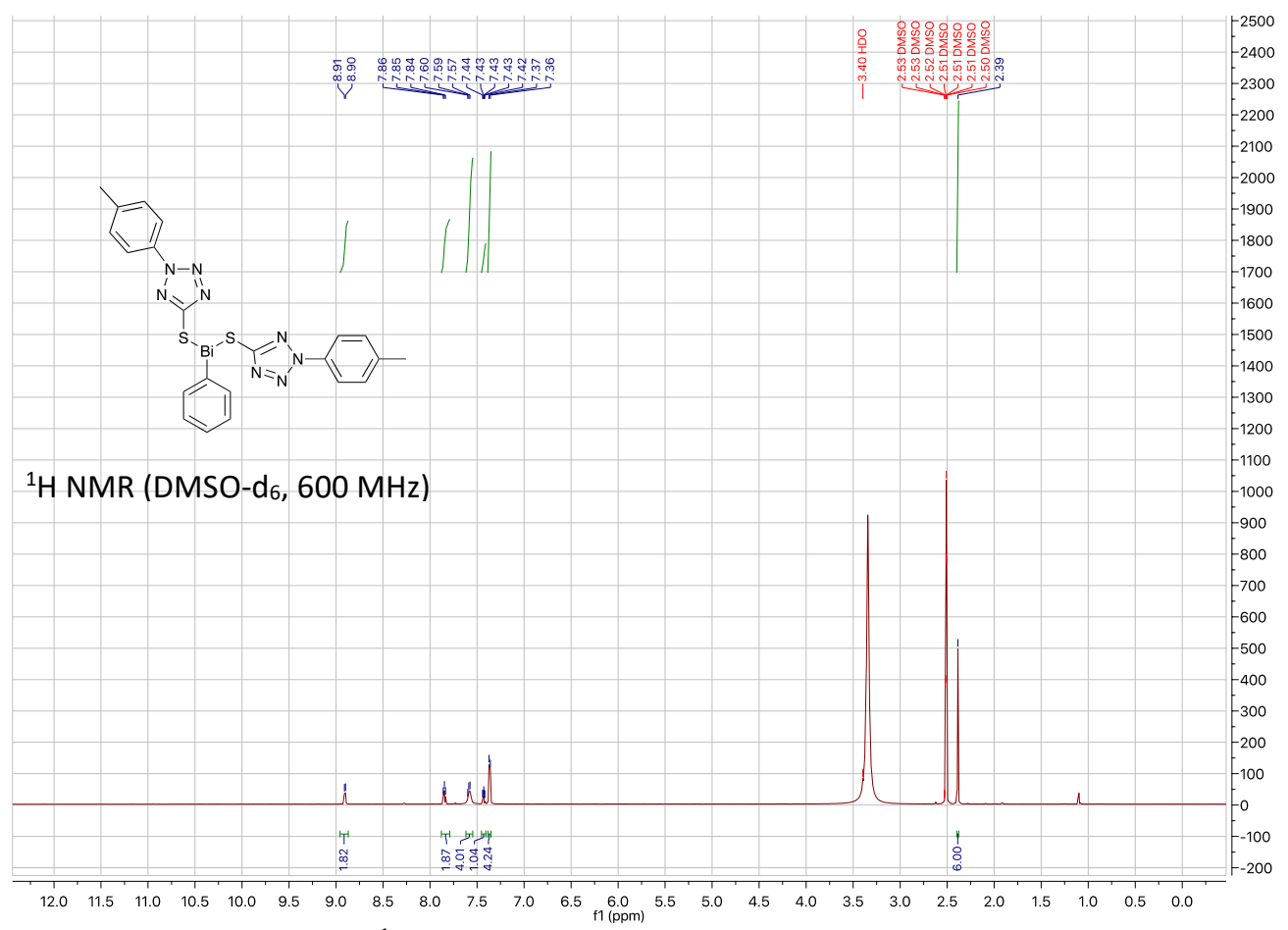

Figure S38. ${ }^{1} \mathrm{H} \mathrm{NMR}$ of $\left[\mathrm{Bi}(\mathrm{Ph})\left(\mathrm{C}_{8} \mathrm{H}_{7} \mathrm{~N}_{4} \mathrm{~S}\right)_{2}\right] 2 \mathbf{2 a}\left(\mathrm{DMSO}-\mathrm{d}_{6}\right)$

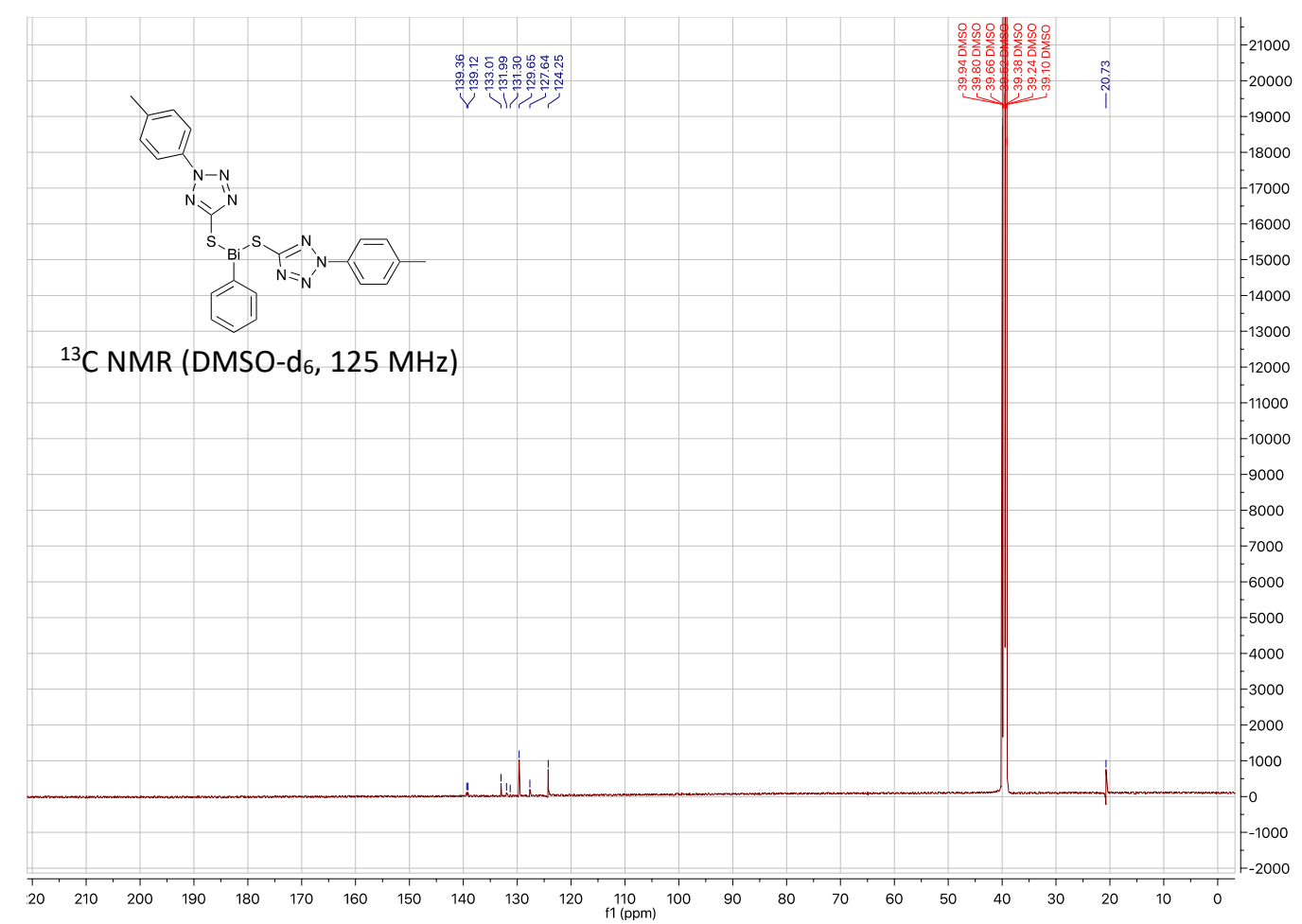

Figure S39. ${ }^{13} \mathrm{C}$ NMR of $\left[\mathrm{Bi}(\mathrm{Ph})\left(\mathrm{C}_{8} \mathrm{H}_{7} \mathrm{~N}_{4} \mathrm{~S}\right)_{2}\right] \mathbf{2 a}\left(\mathrm{DMSO}-\mathrm{d}_{6}\right)$ 


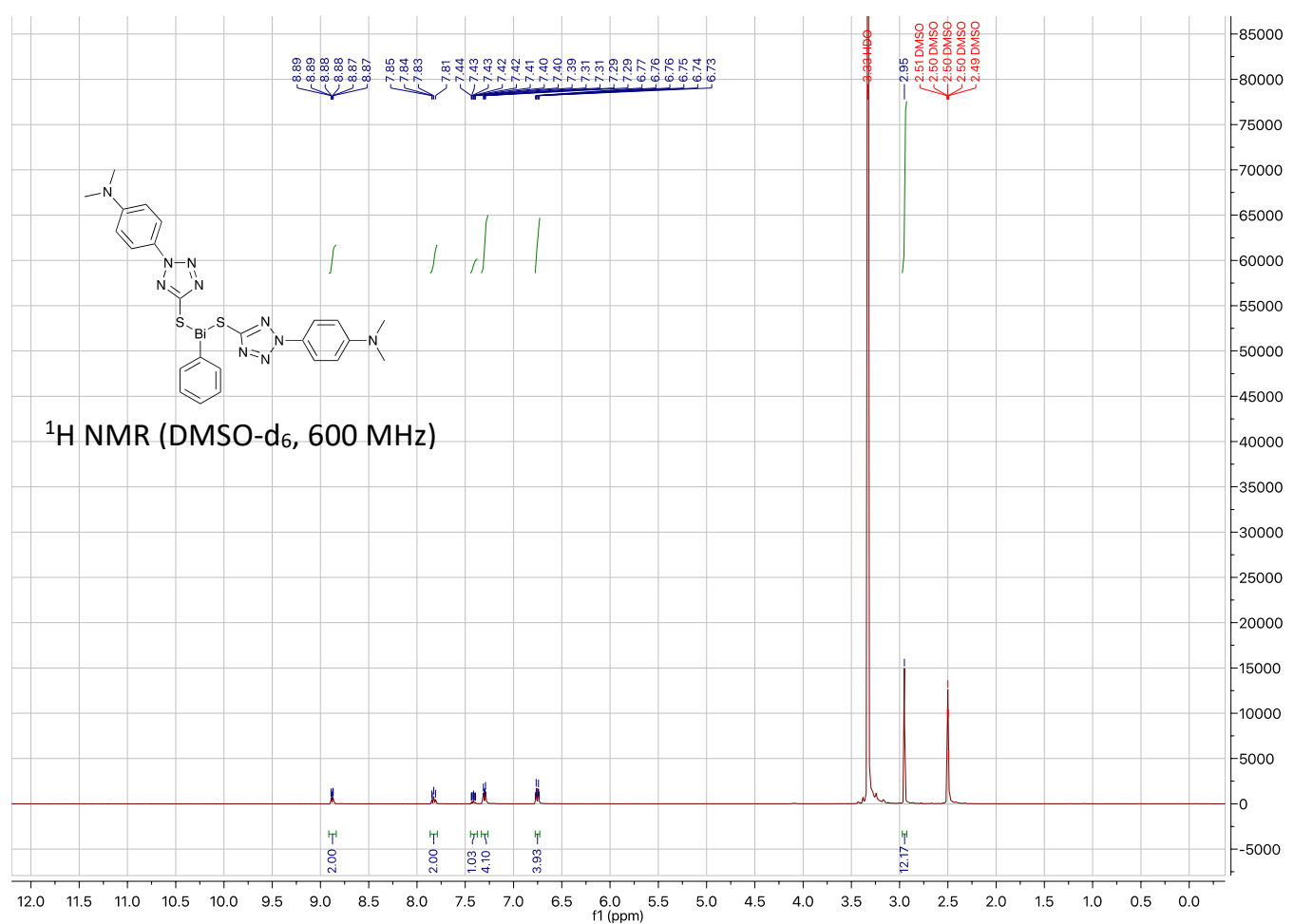

Figure S40. ${ }^{1} \mathrm{H} \mathrm{NMR}$ of $\left[\mathrm{Bi}(\mathrm{Ph})\left(\mathrm{C}_{9} \mathrm{H}_{10} \mathrm{~N}_{5} \mathrm{~S}\right)_{2}\right] \mathbf{2 b}\left(\mathrm{DMSO}_{-} \mathrm{d}_{6}\right)$

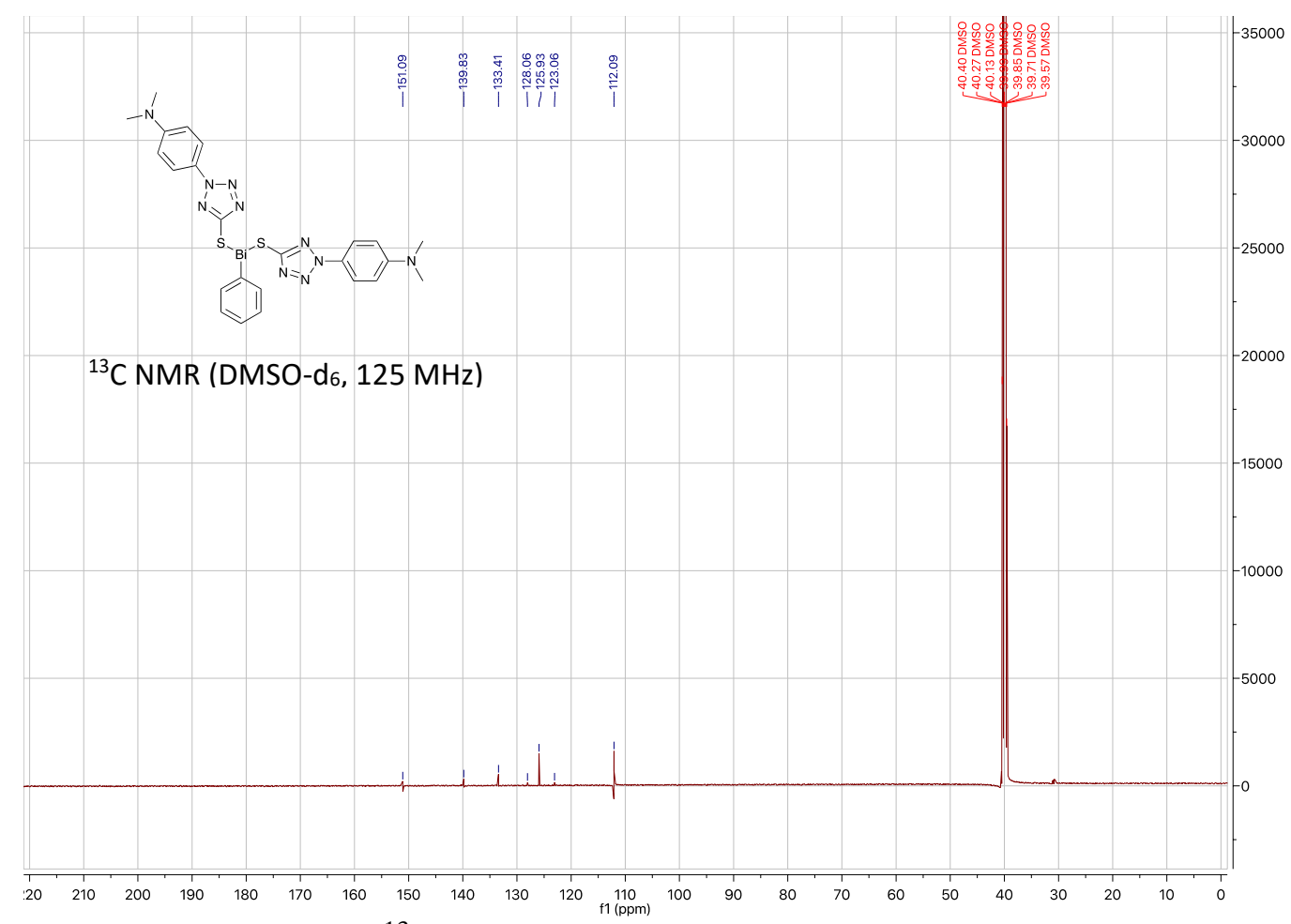

Figure S41. ${ }^{13} \mathrm{C}$ NMR of $\left[\mathrm{Bi}(\mathrm{Ph})\left(\mathrm{C}_{9} \mathrm{H}_{10} \mathrm{~N}_{5} \mathrm{~S}\right)_{2}\right] \mathbf{2 b}\left(\mathrm{DMSO}-\mathrm{d}_{6}\right)$ 


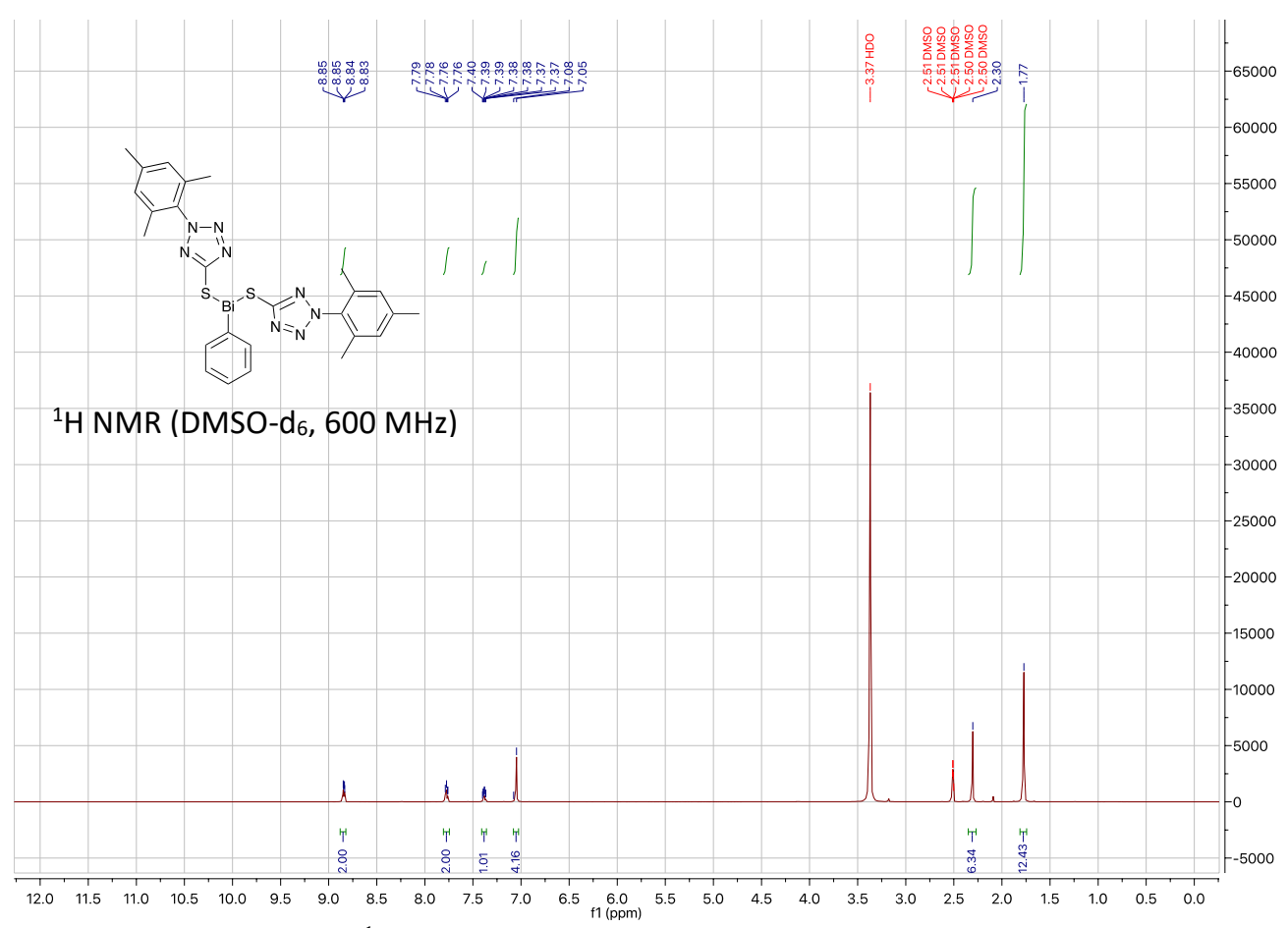

Figure S42. ${ }^{1} \mathrm{H}$ NMR of $\left[\mathrm{Bi}(\mathrm{Ph})\left(\mathrm{C}_{10} \mathrm{H}_{11} \mathrm{~N}_{4} \mathrm{~S}\right)_{2}\right] 2 \mathbf{2 c}\left(\mathrm{DMSO}-\mathrm{d}_{6}\right)$

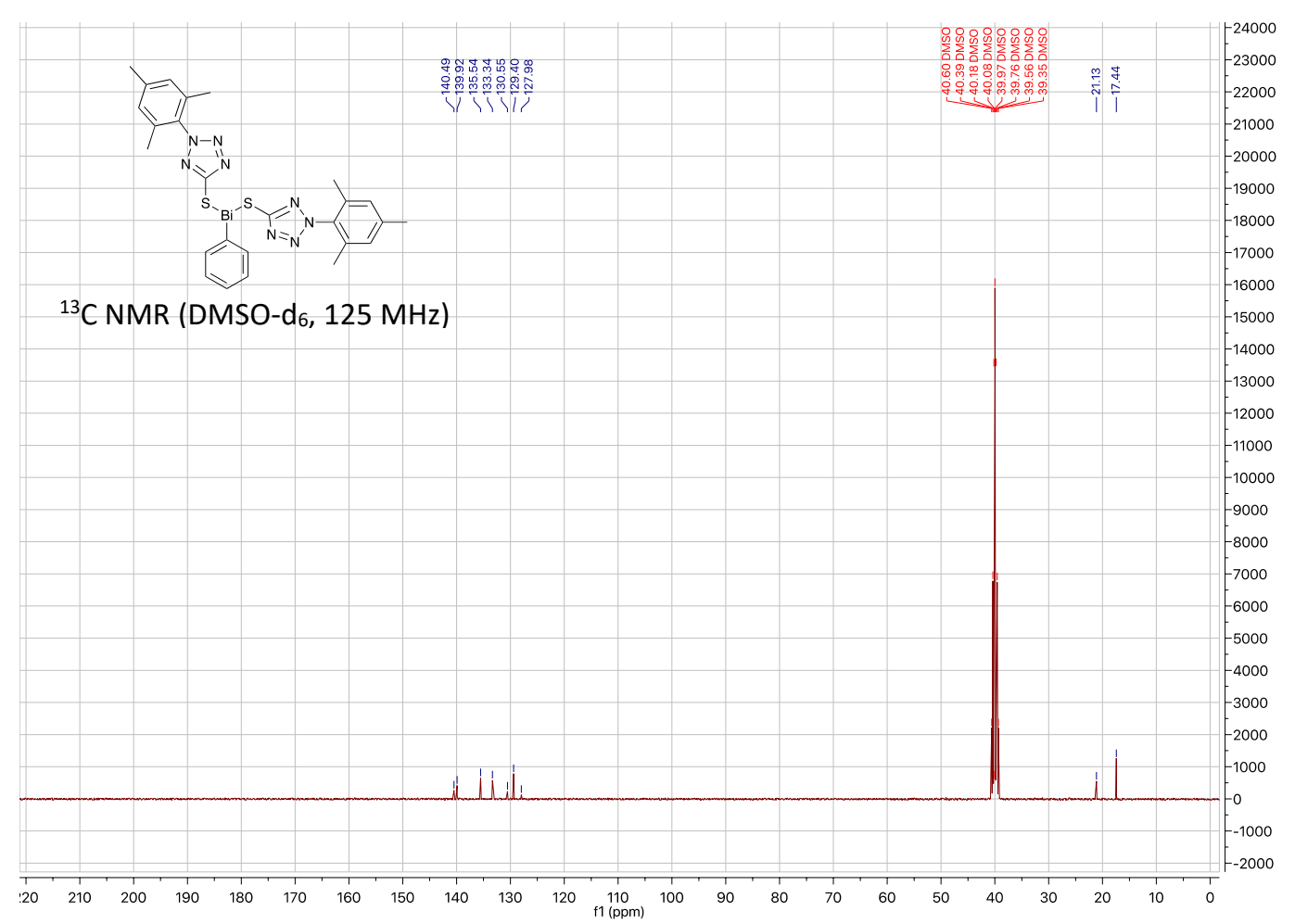

Figure S43. ${ }^{13} \mathrm{C}$ NMR of $\left[\mathrm{Bi}(\mathrm{Ph})\left(\mathrm{C}_{10} \mathrm{H}_{11} \mathrm{~N}_{4} \mathrm{~S}\right)_{2}\right] 2 \mathrm{c}\left(\mathrm{DMSO}-\mathrm{d}_{6}\right)$ 


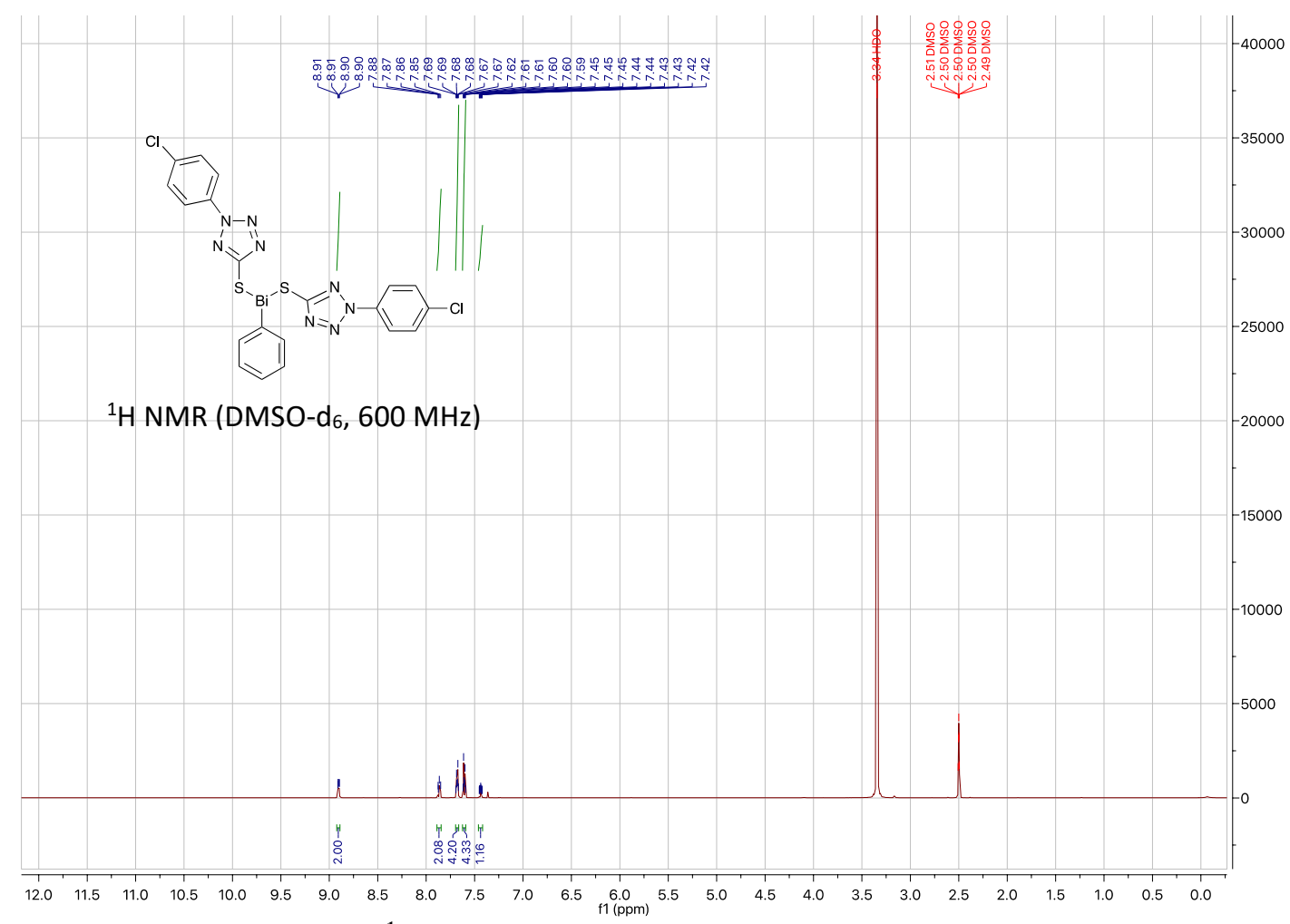

Figure S44. ${ }^{1} \mathrm{H}$ NMR of $\left[\mathrm{Bi}(\mathrm{Ph})\left(\mathrm{C}_{7} \mathrm{H}_{4} \mathrm{~N}_{4} \mathrm{ClS}\right)_{2}\right]$ 2d $\left(\mathrm{DMSO}-\mathrm{d}_{6}\right)$

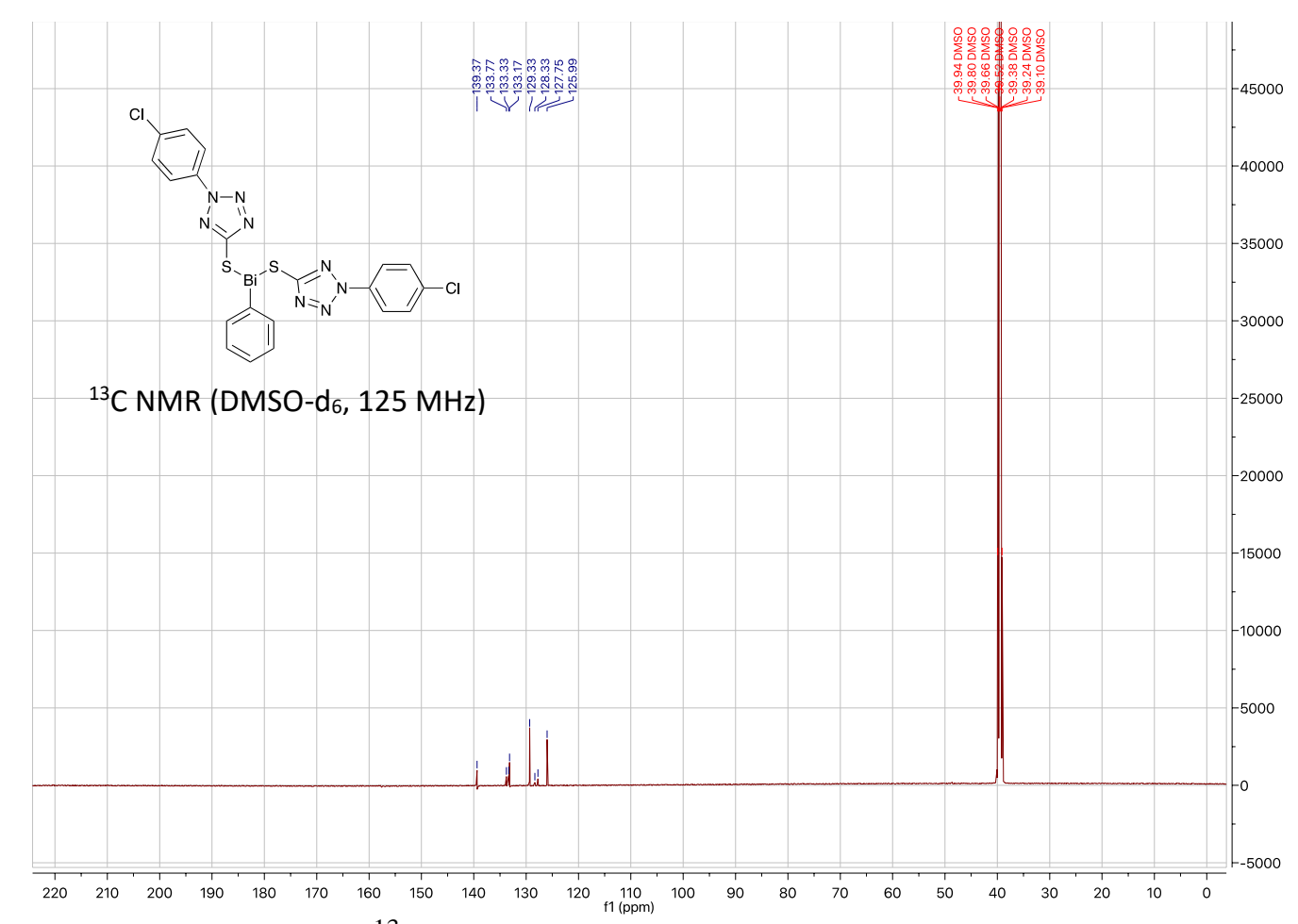

Figure S45. ${ }^{13} \mathrm{C}$ NMR of $\left[\mathrm{Bi}(\mathrm{Ph})\left(\mathrm{C}_{7} \mathrm{H}_{4} \mathrm{~N}_{4} \mathrm{ClS}\right)_{2}\right] \mathbf{2 d}\left(\mathrm{DMSO}_{-} \mathrm{d}_{6}\right)$ 


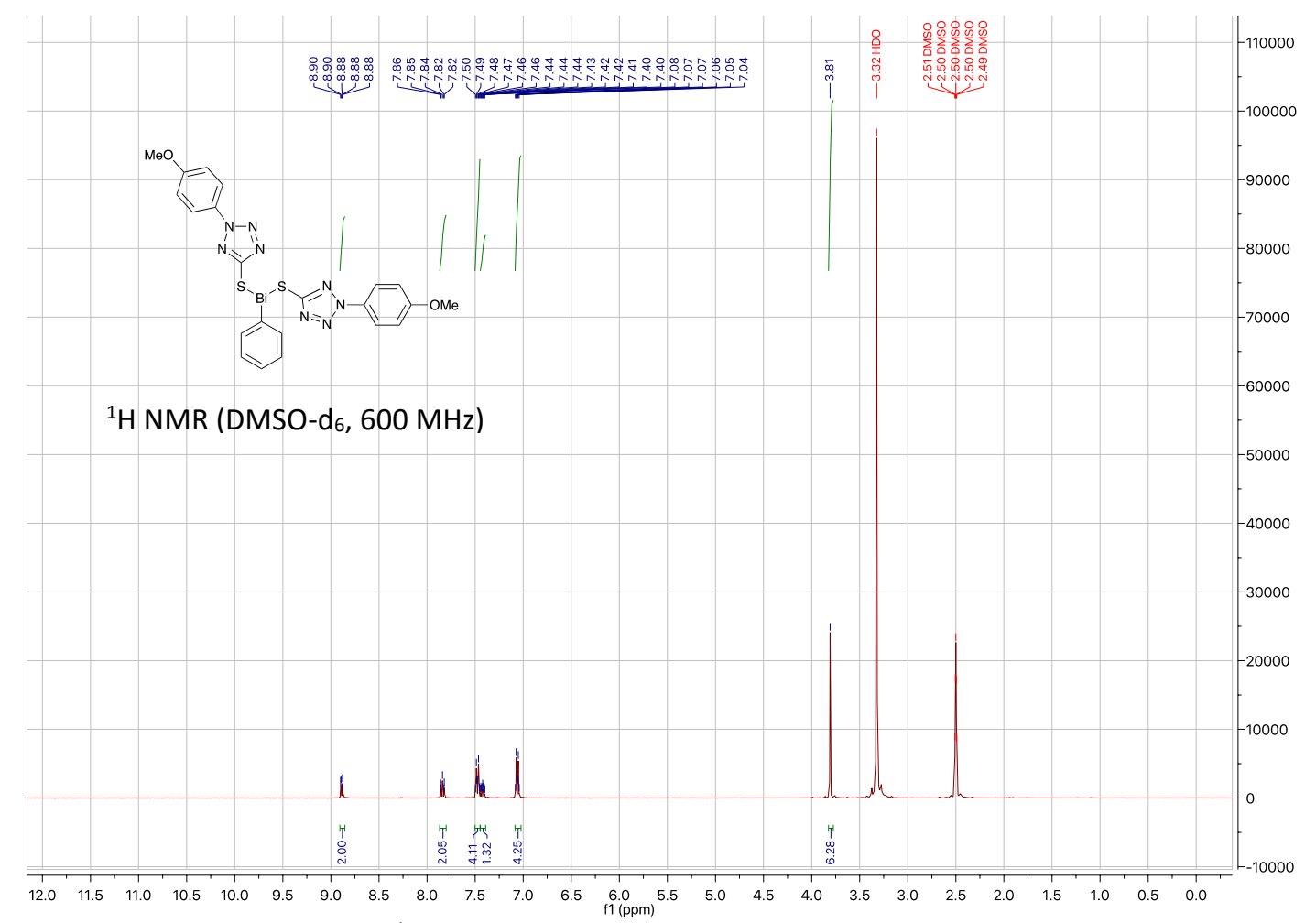

Figure S46. ${ }^{1} \mathrm{H}$ NMR of $\left[\mathrm{Bi}(\mathrm{Ph})\left(\mathrm{C}_{8} \mathrm{H}_{7} \mathrm{~N}_{4} \mathrm{OS}\right)_{2}\right] \mathbf{2 e}\left(\mathrm{DMSO}_{-} \mathrm{d}_{6}\right)$

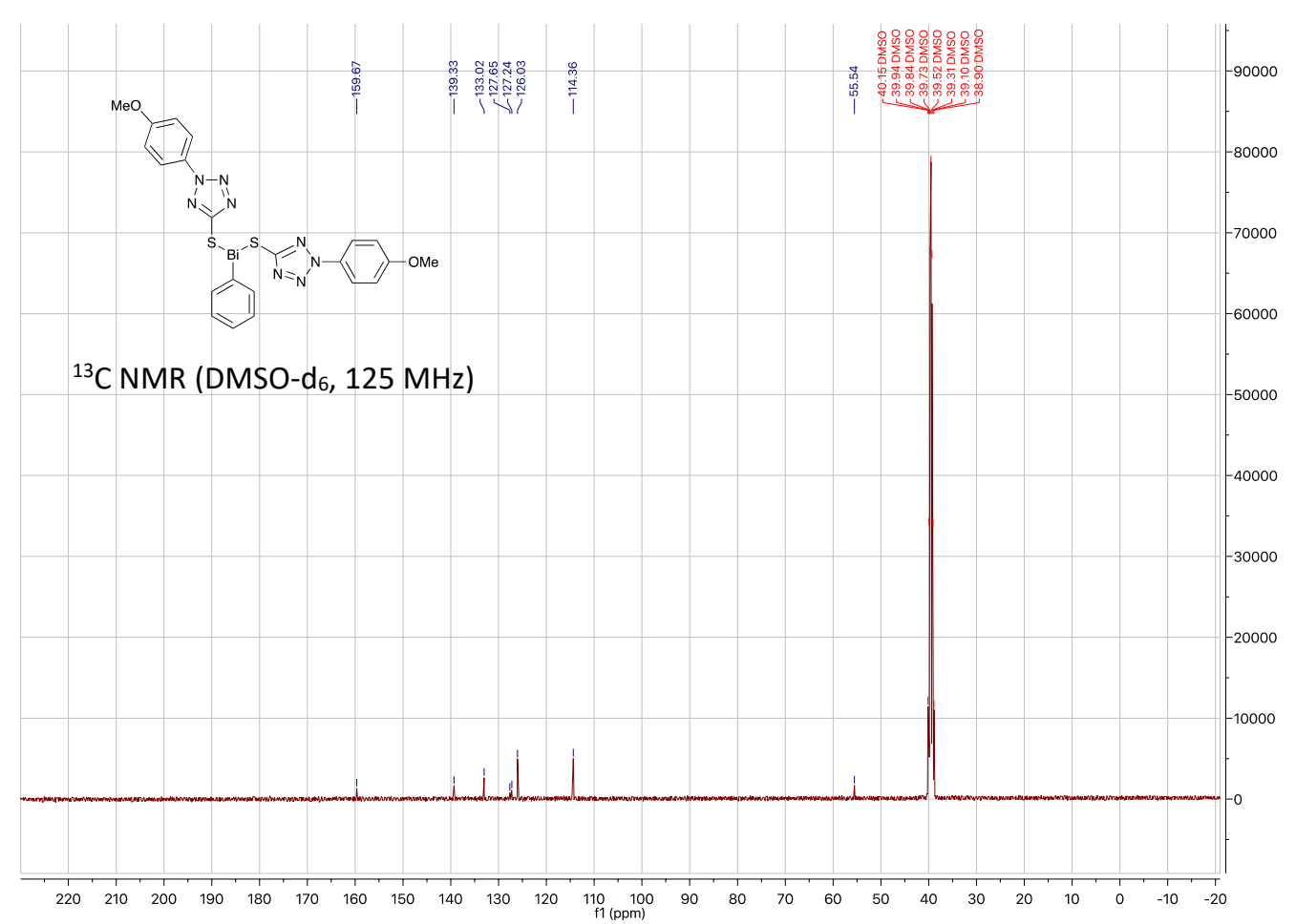

Figure S47. ${ }^{13} \mathrm{C}$ NMR of $\left[\mathrm{Bi}(\mathrm{Ph})\left(\mathrm{C}_{8} \mathrm{H}_{7} \mathrm{~N}_{4} \mathrm{OS}\right)_{2}\right] \mathbf{2 e}\left(\mathrm{DMSO}-\mathrm{d}_{6}\right)$ 


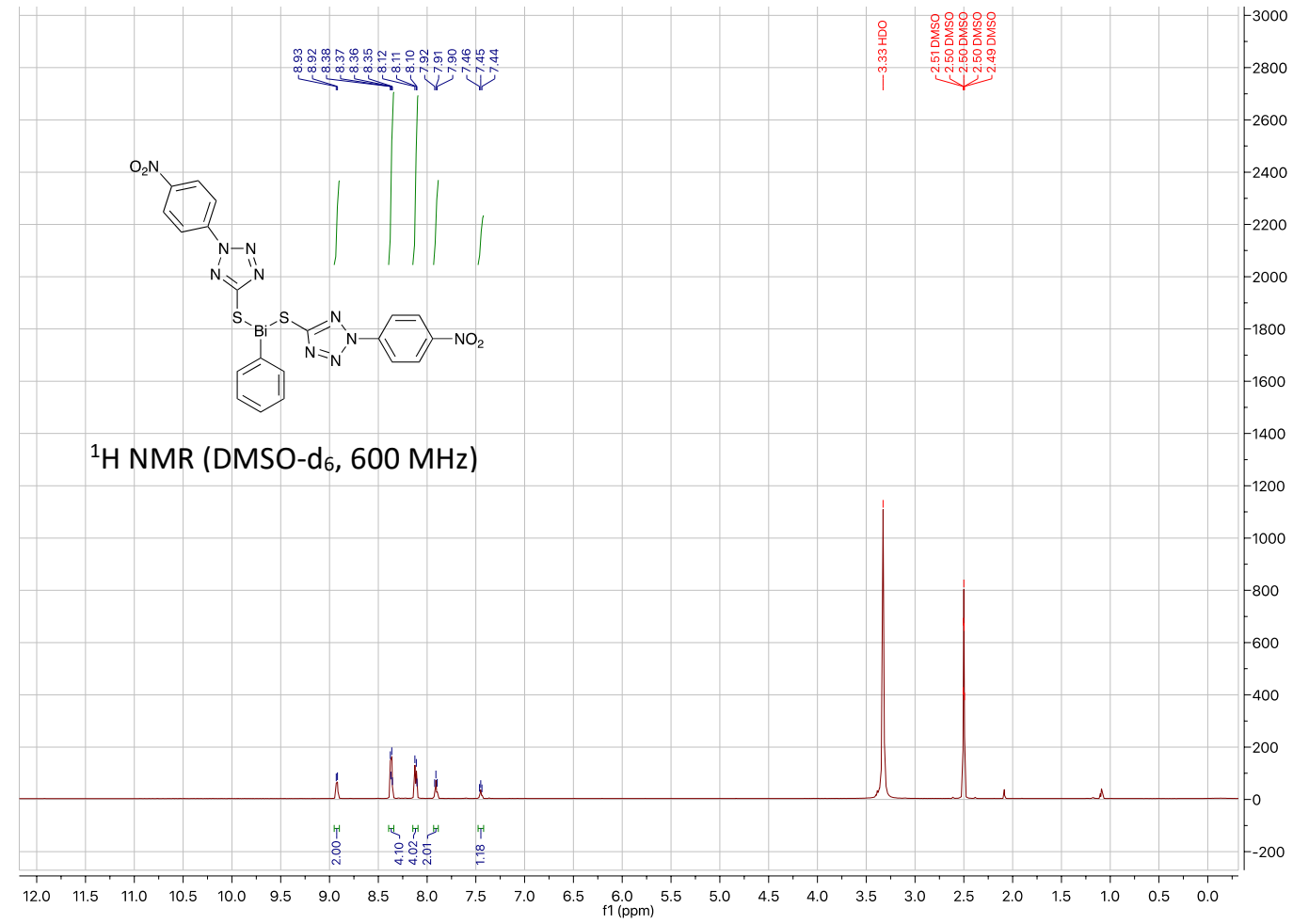

Figure S48. ${ }^{1} \mathrm{H}$ NMR of $\left[\mathrm{Bi}(\mathrm{Ph})\left(\mathrm{C}_{7} \mathrm{H}_{4} \mathrm{~N}_{5} \mathrm{O}_{2} \mathrm{~S}\right)_{2}\right] \mathbf{2 f}\left(\mathrm{DMSO}-\mathrm{d}_{6}\right)$

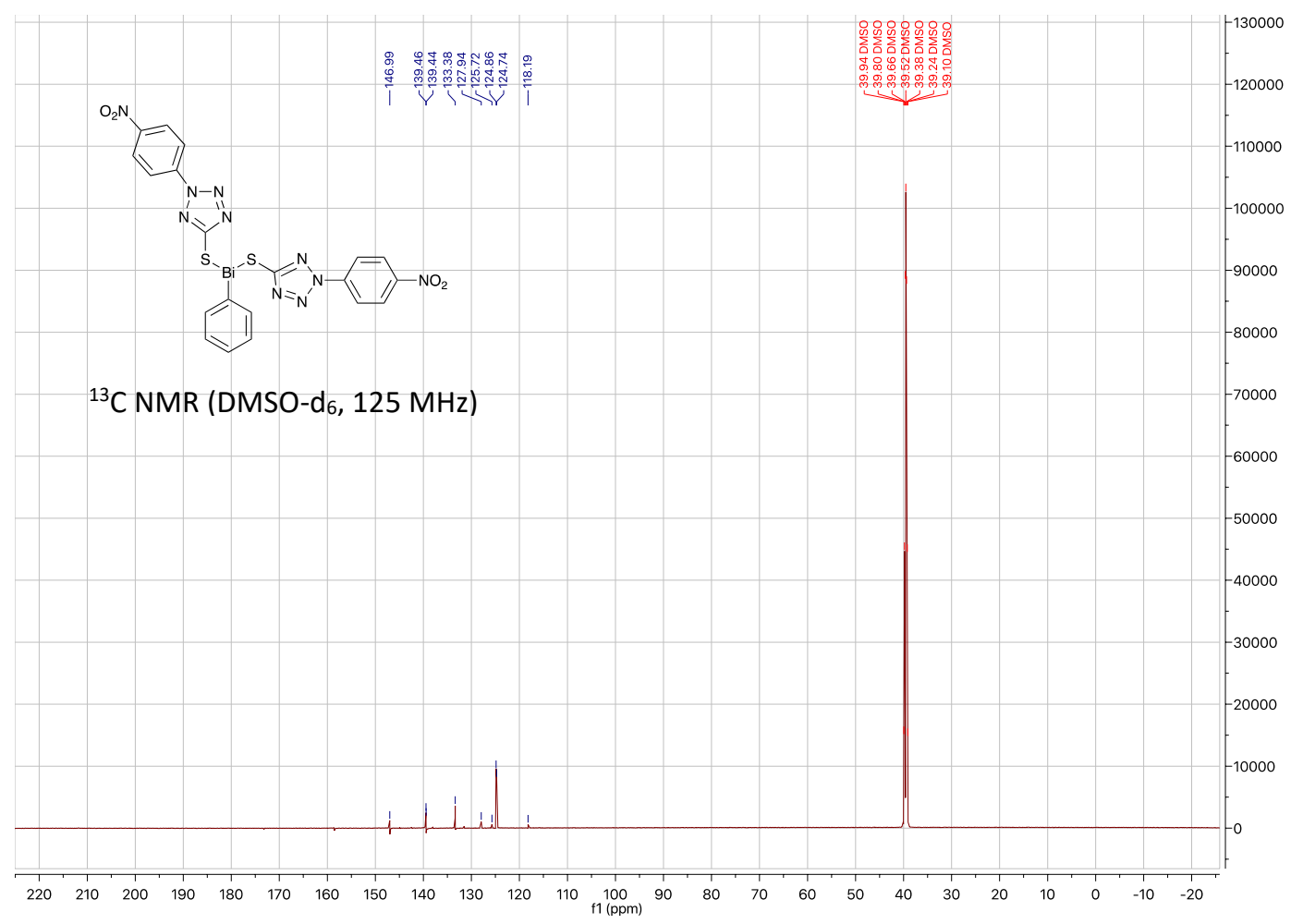

Figure S49. ${ }^{13} \mathrm{C}$ NMR of $\left[\mathrm{Bi}(\mathrm{Ph})\left(\mathrm{C}_{7} \mathrm{H}_{4} \mathrm{~N}_{5} \mathrm{O}_{2} \mathrm{~S}\right)_{2}\right] 2 \mathbf{f}\left(\mathrm{DMSO}-\mathrm{d}_{6}\right)$ 


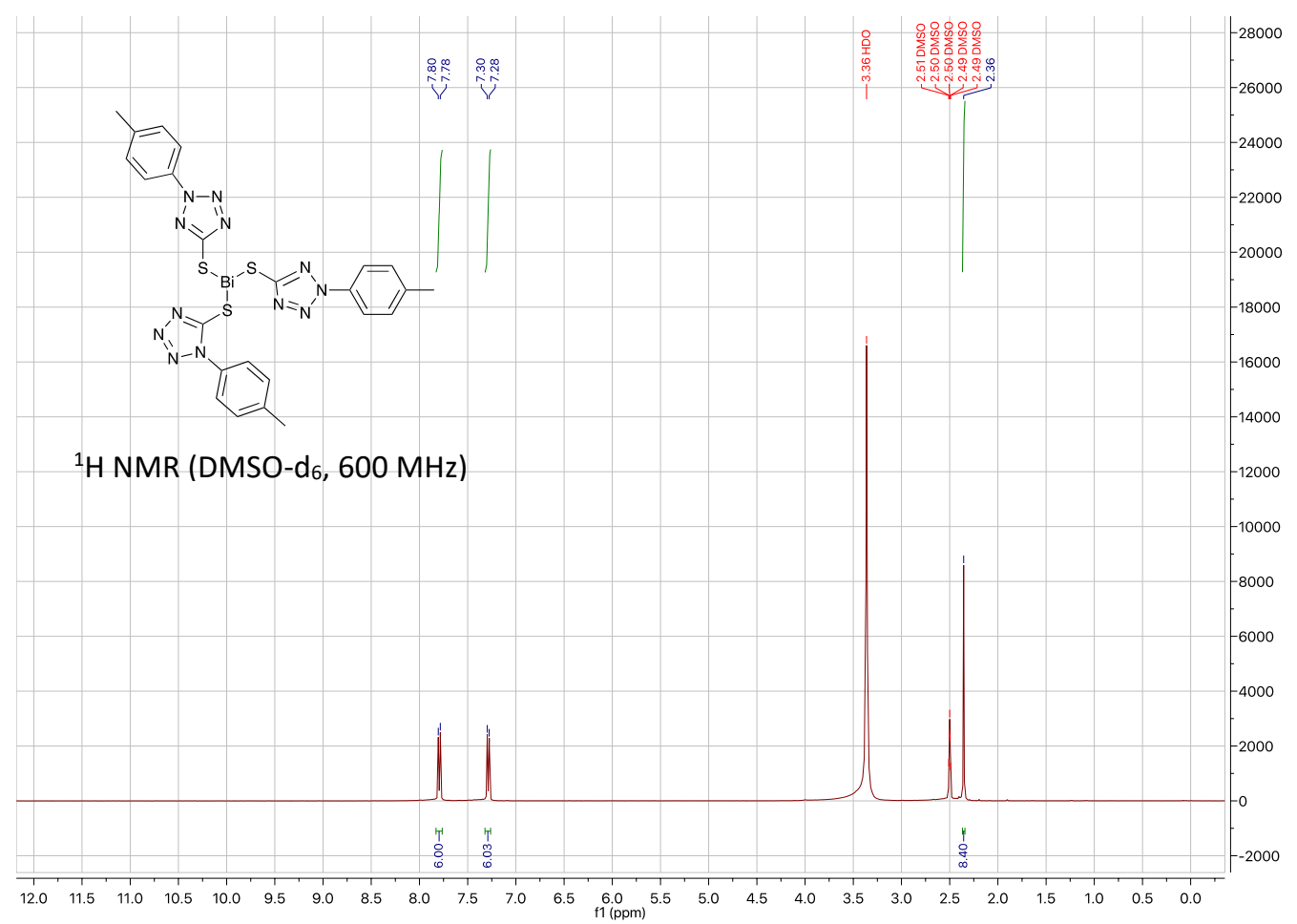

Figure S50. ${ }^{1} \mathrm{H}$ NMR of $\left[\mathrm{Bi}\left(\mathrm{C}_{8} \mathrm{H}_{7} \mathrm{~N}_{4} \mathrm{~S}\right)_{3}\right]$ 3a $\left(\mathrm{DMSO}-\mathrm{d}_{6}\right)$

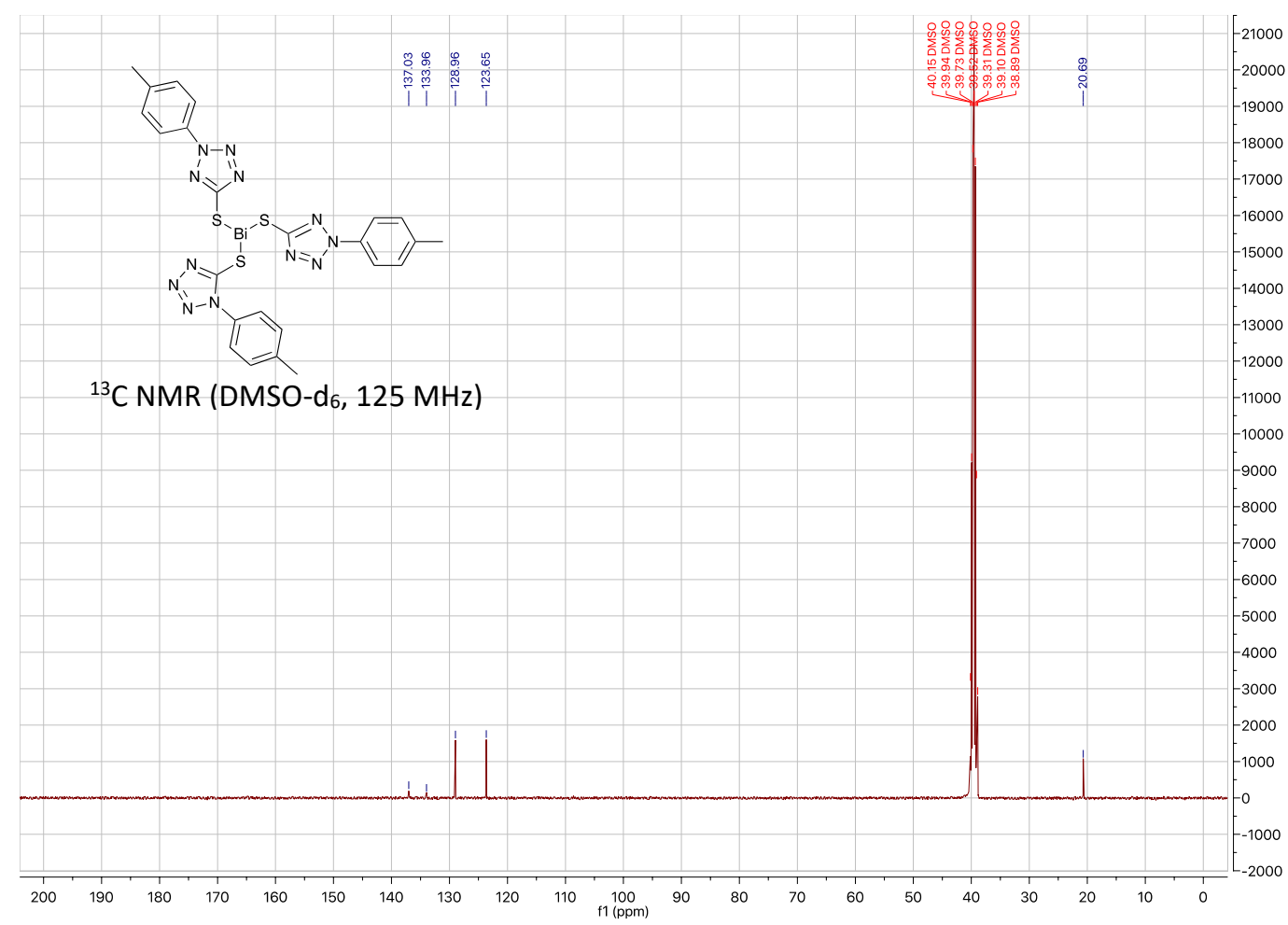

Figure S51. ${ }^{13} \mathrm{C}$ NMR of $\left[\mathrm{Bi}\left(\mathrm{C}_{8} \mathrm{H}_{7} \mathrm{~N}_{4} \mathrm{~S}\right)_{3}\right]$ 3a $\left(\mathrm{DMSO}-\mathrm{d}_{6}\right)$ 


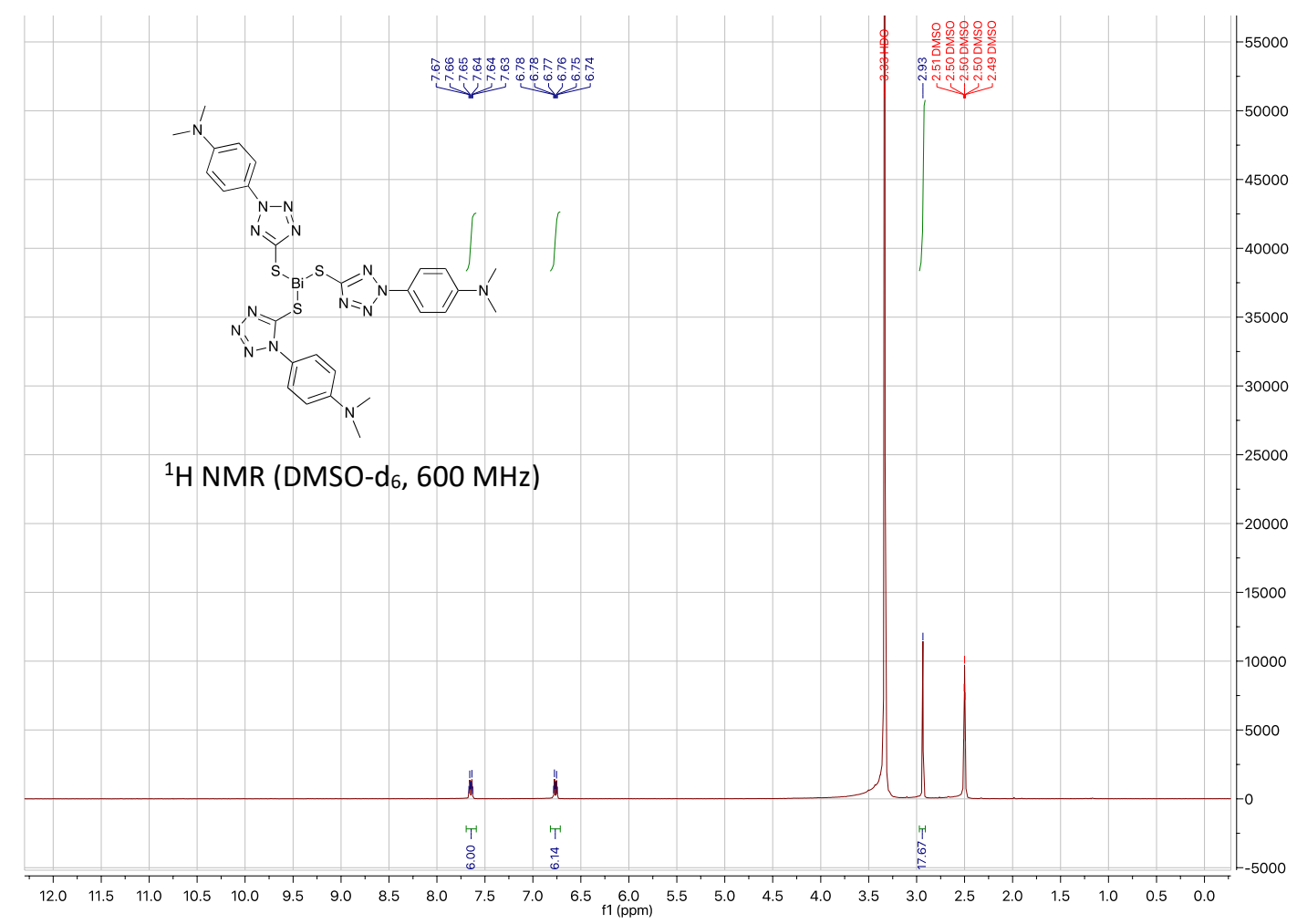

Figure S52. ${ }^{1} \mathrm{H} \mathrm{NMR}$ of $\left[\mathrm{Bi}\left(\mathrm{C}_{9} \mathrm{H}_{10} \mathrm{~N}_{5} \mathrm{~S}\right)_{3}\right]$ 3b $\left(\mathrm{DMSO}-\mathrm{d}_{6}\right)$

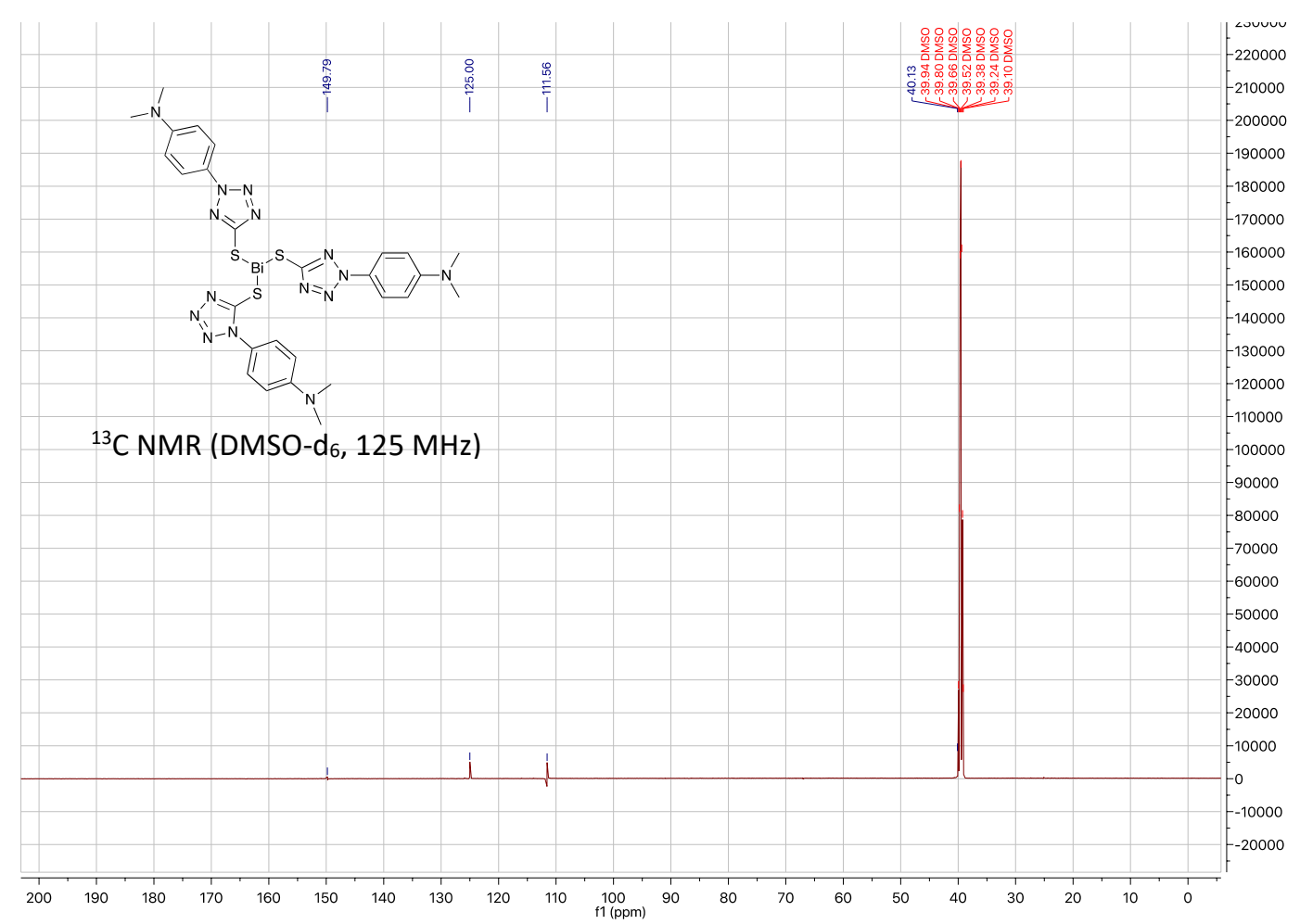

Figure S53. ${ }^{13} \mathrm{C}$ NMR of $\left[\mathrm{Bi}\left(\mathrm{C}_{9} \mathrm{H}_{10} \mathrm{~N}_{5} \mathrm{~S}\right)_{3}\right] \mathbf{3 b}\left(\mathrm{DMSO}-\mathrm{d}_{6}\right)$ 


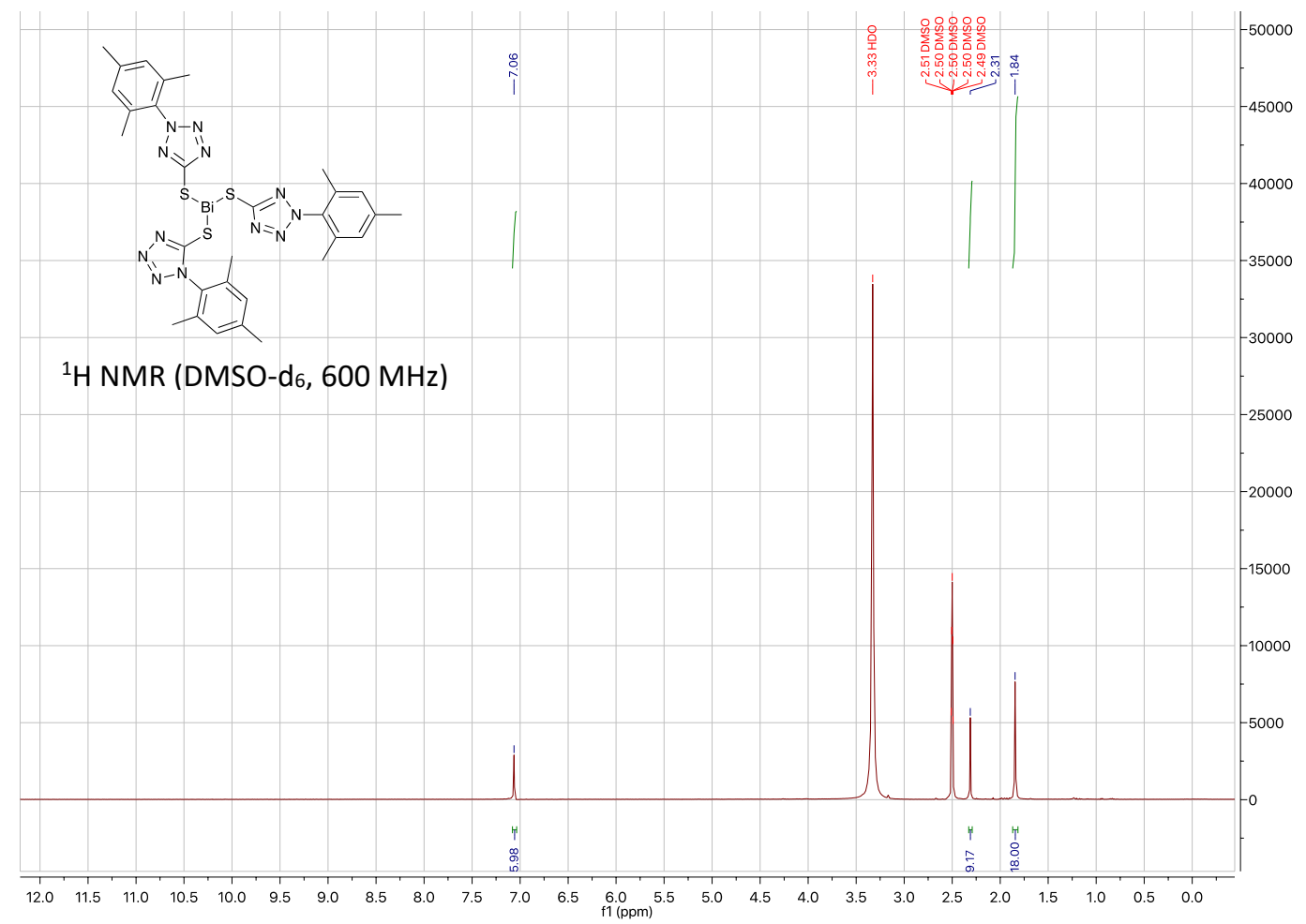

Figure S54. ${ }^{1} \mathrm{H}$ NMR of $\left[\mathrm{Bi}\left(\mathrm{C}_{10} \mathrm{H}_{11} \mathrm{~N}_{4} \mathrm{~S}\right)_{3}\right]$ 3c $\left(\mathrm{DMSO}-\mathrm{d}_{6}\right)$

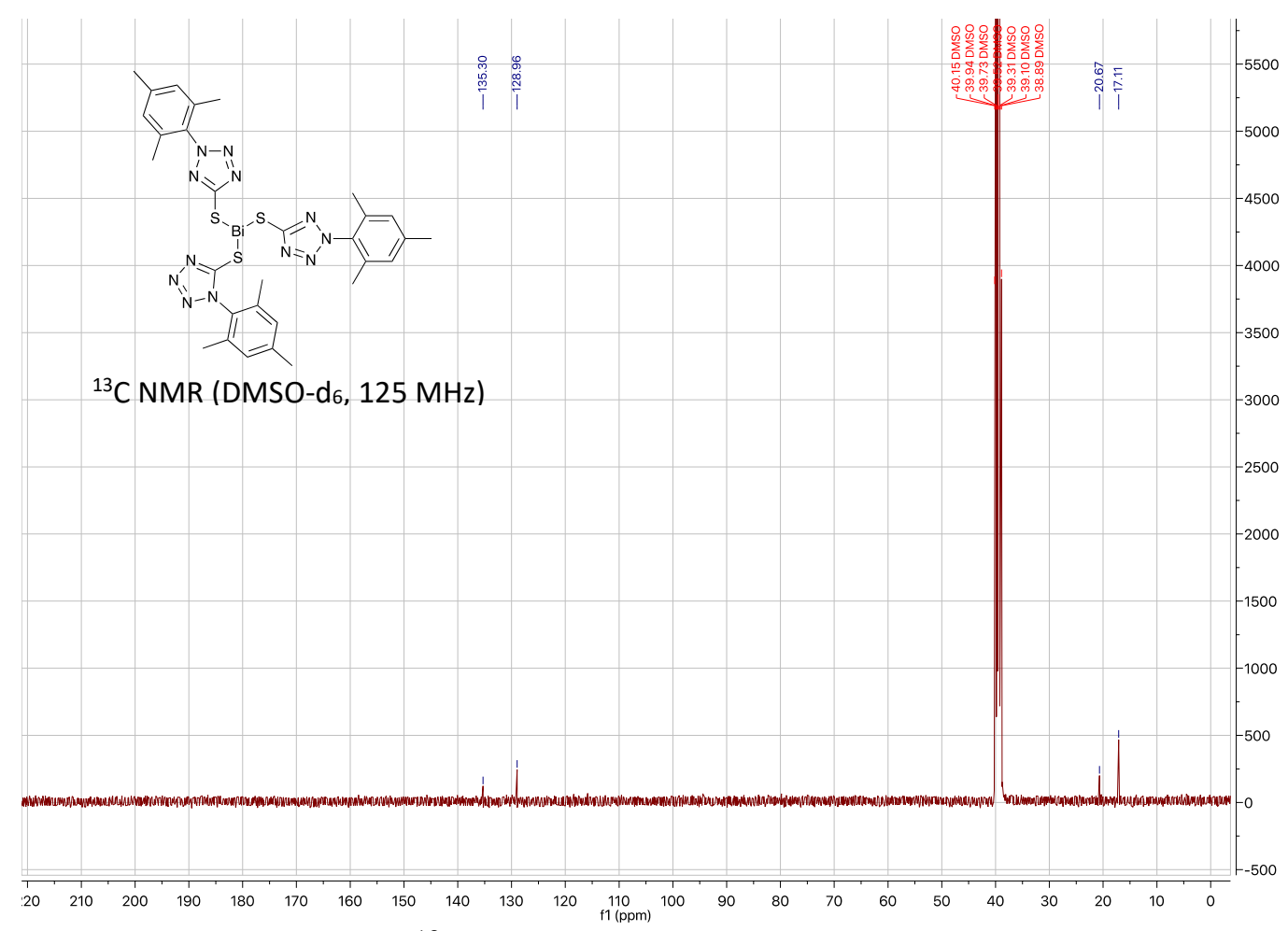

Figure S55. ${ }^{13} \mathrm{C}$ NMR of $\left[\mathrm{Bi}\left(\mathrm{C}_{10} \mathrm{H}_{11} \mathrm{~N}_{4} \mathrm{~S}\right)_{3}\right]$ 3c $\left(\mathrm{DMSO}_{-} \mathrm{d}_{6}\right)$ 


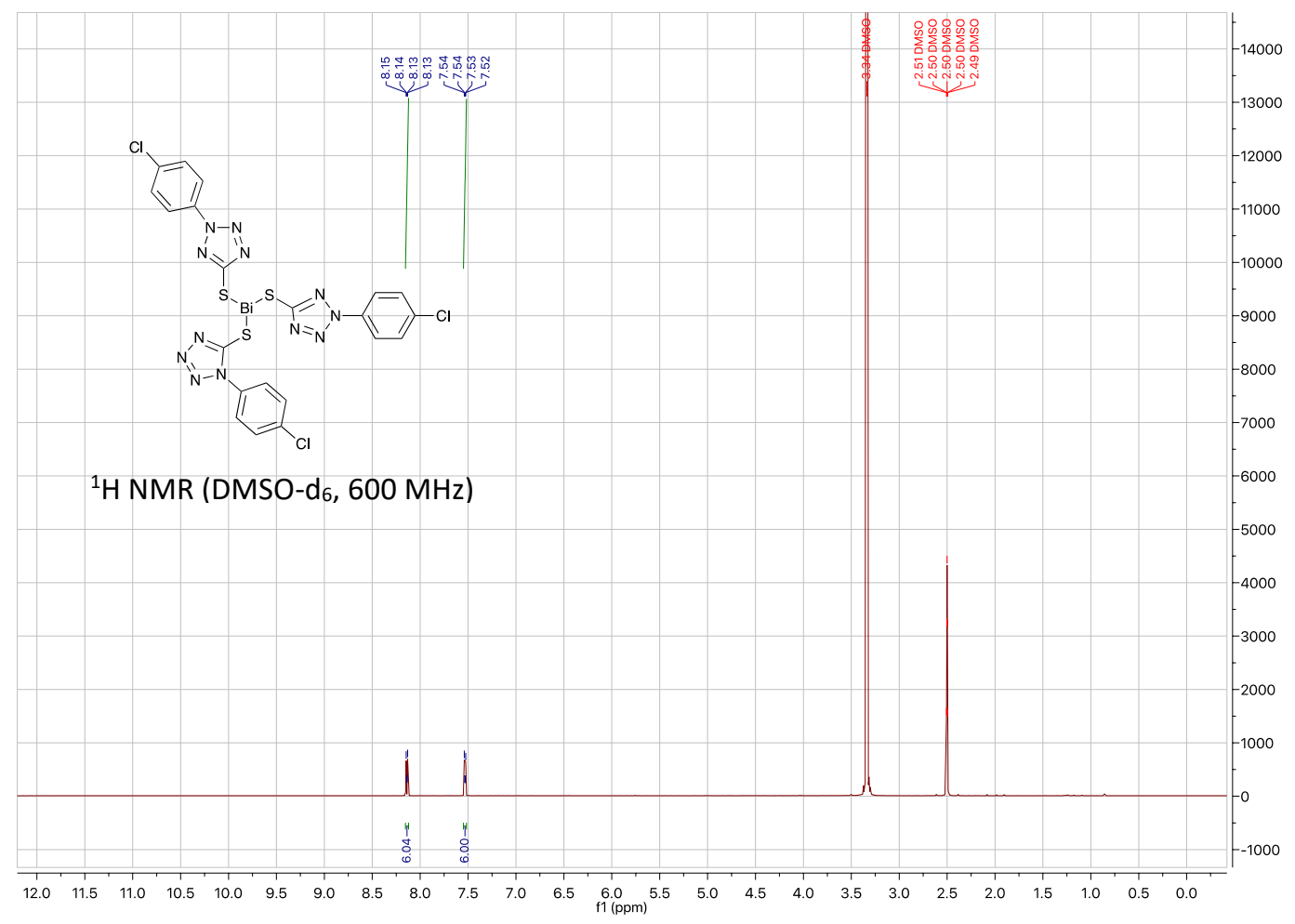

Figure S56. ${ }^{1} \mathrm{H}$ NMR of $\left[\mathrm{Bi}\left(\mathrm{C}_{7} \mathrm{H}_{4} \mathrm{~N}_{4} \mathrm{ClS}\right)_{3}\right]$ 3d $\left(\mathrm{DMSO}-\mathrm{d}_{6}\right)$

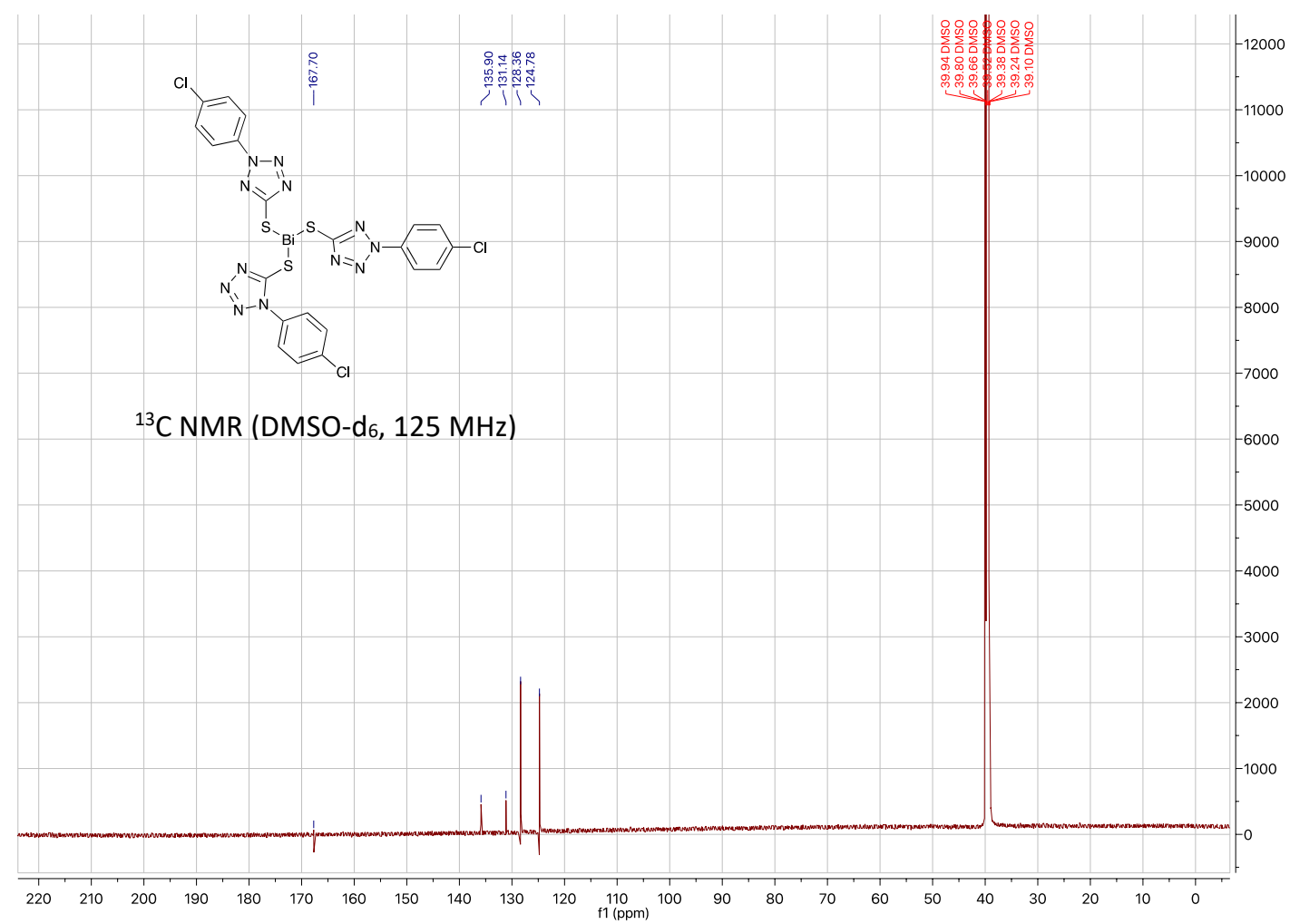

Figure S57. ${ }^{13} \mathrm{C}$ NMR of $\left[\mathrm{Bi}\left(\mathrm{C}_{7} \mathrm{H}_{4} \mathrm{~N}_{4} \mathrm{ClS}\right)_{3}\right]$ 3d (DMSO- $\left.\mathrm{d}_{6}\right)$ 


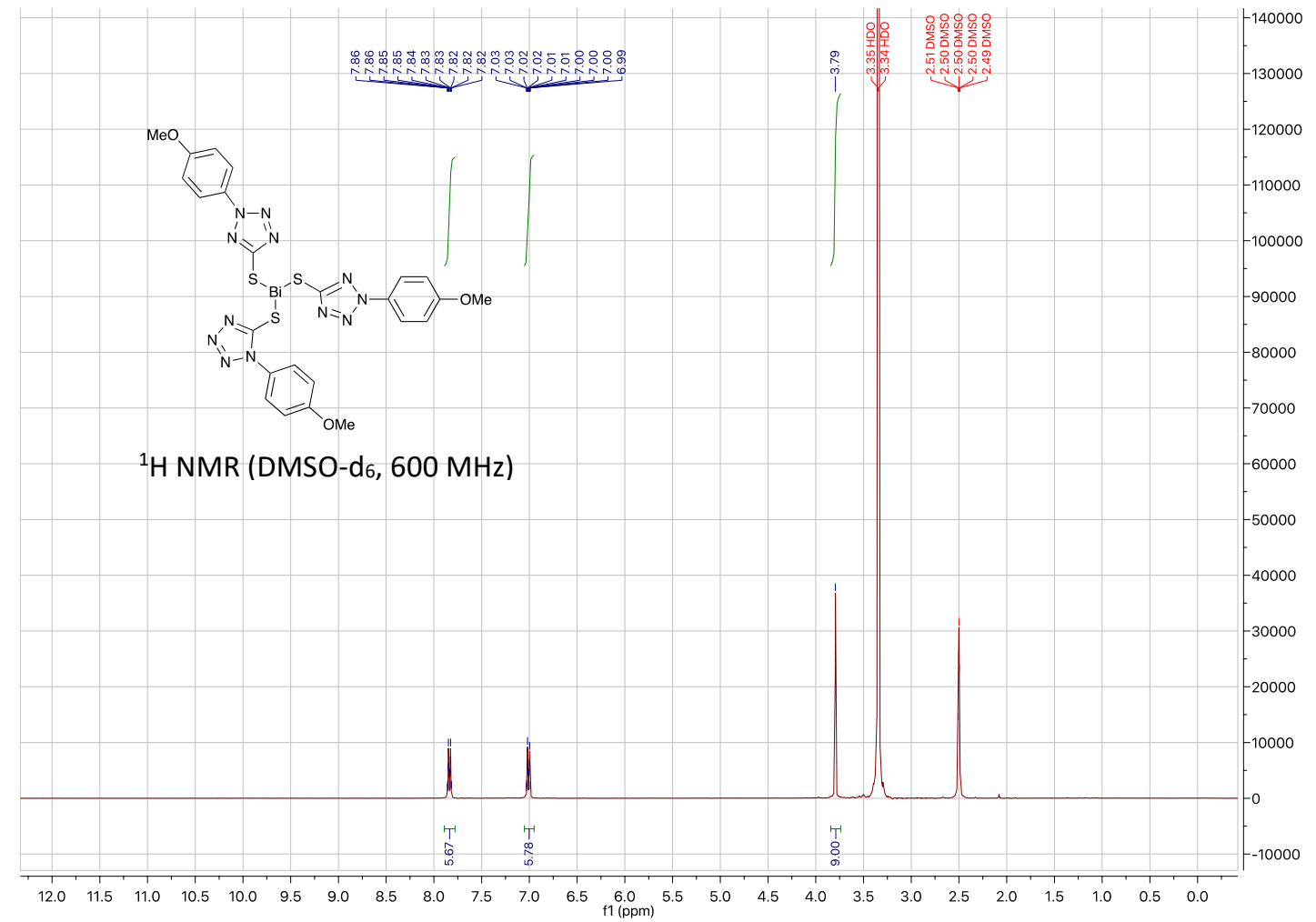

Figure S58. ${ }^{1} \mathrm{H}$ NMR of $\left[\mathrm{Bi}\left(\mathrm{C}_{8} \mathrm{H}_{7} \mathrm{~N}_{4} \mathrm{OS}\right)_{3}\right]$ 3e $\left(\mathrm{DMSO}-\mathrm{d}_{6}\right)$

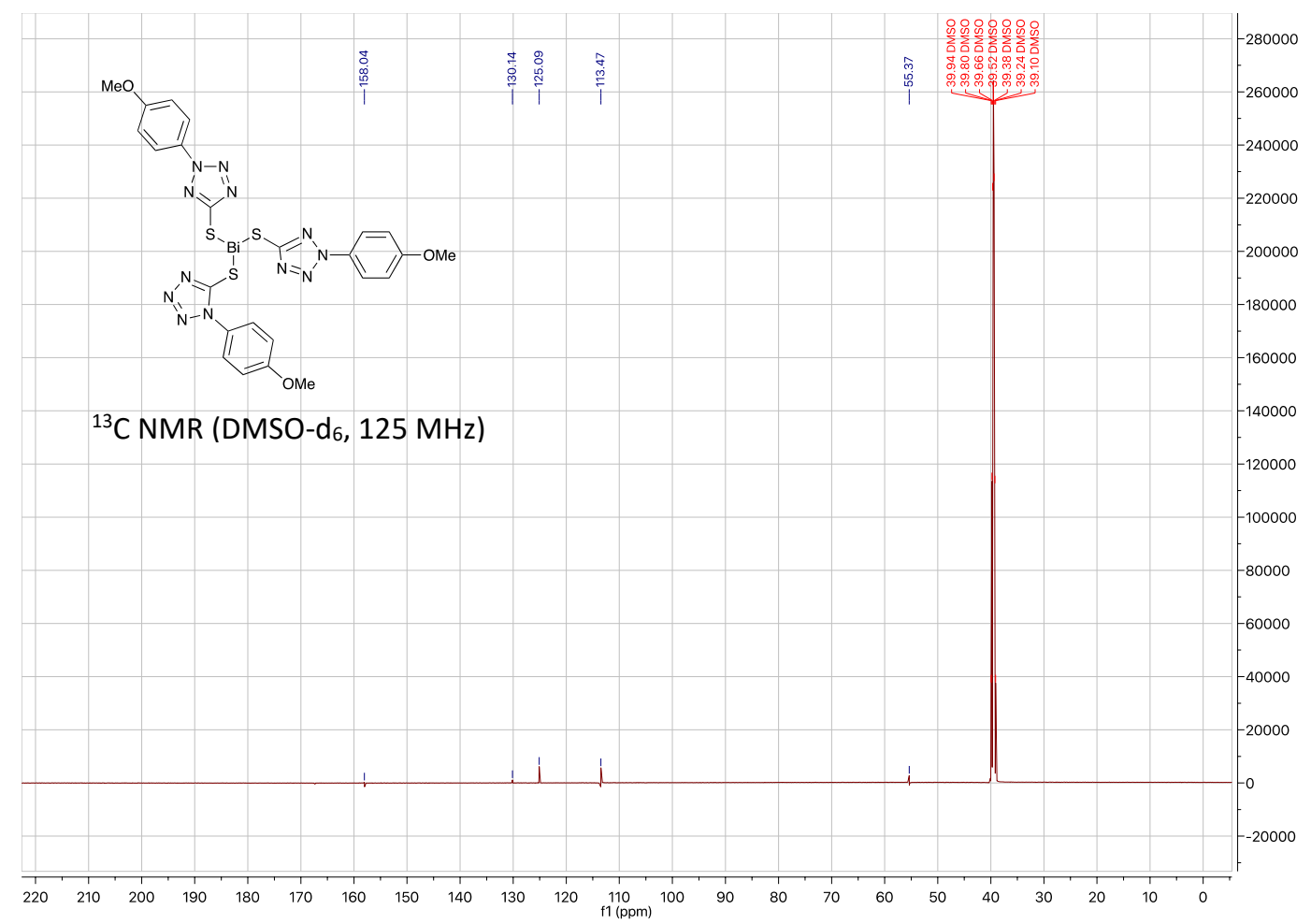

Figure S59. ${ }^{13} \mathrm{C}$ NMR of $\left[\mathrm{Bi}\left(\mathrm{C}_{8} \mathrm{H}_{7} \mathrm{~N}_{4} \mathrm{OS}\right)_{3}\right]$ 3e $\left(\mathrm{DMSO}-\mathrm{d}_{6}\right)$ 


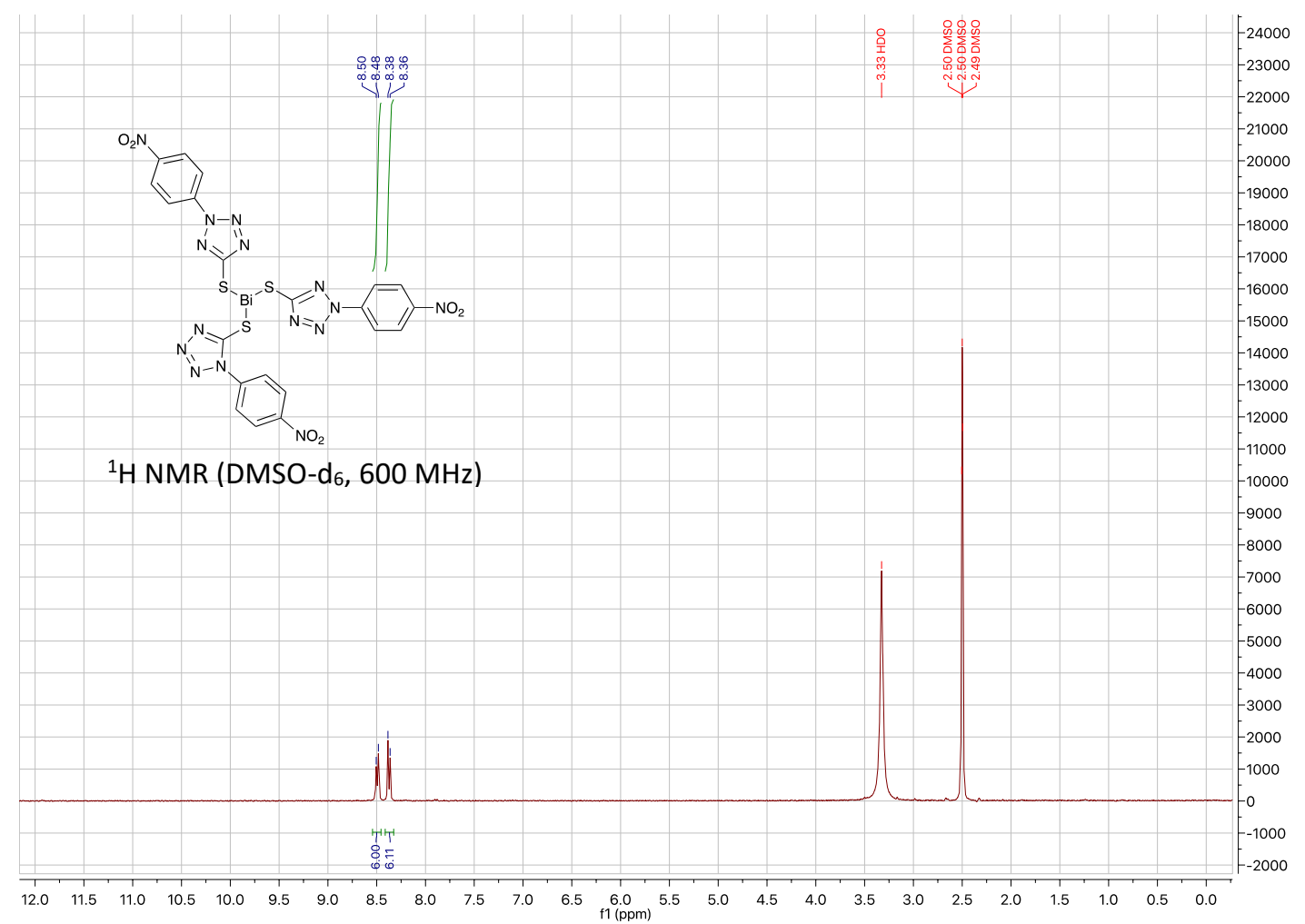

Figure S60. ${ }^{1} \mathrm{H}$ NMR of $\left[\mathrm{Bi}\left(\mathrm{C}_{7} \mathrm{H}_{4} \mathrm{~N}_{5} \mathrm{O}_{2} \mathrm{~S}\right)_{3}\right]$ 3f $\left(\mathrm{DMSO}-\mathrm{d}_{6}\right)$

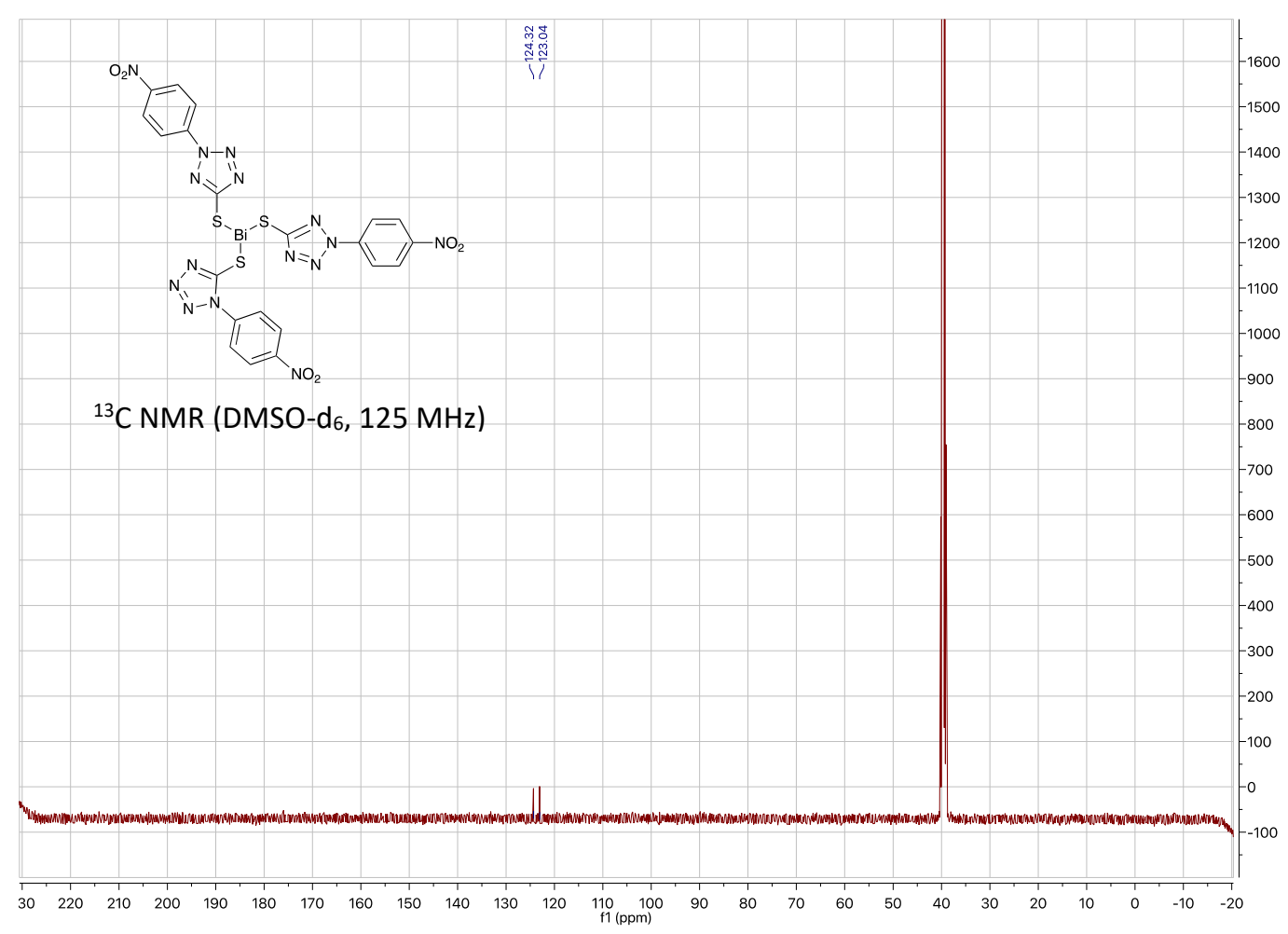

Figure S61. ${ }^{13} \mathrm{C}$ NMR of $\left[\mathrm{Bi}\left(\mathrm{C}_{7} \mathrm{H}_{4} \mathrm{~N}_{5} \mathrm{O}_{2} \mathrm{~S}\right)_{3}\right]$ 3f $\left(\mathrm{DMSO}-\mathrm{d}_{6}\right)$ 


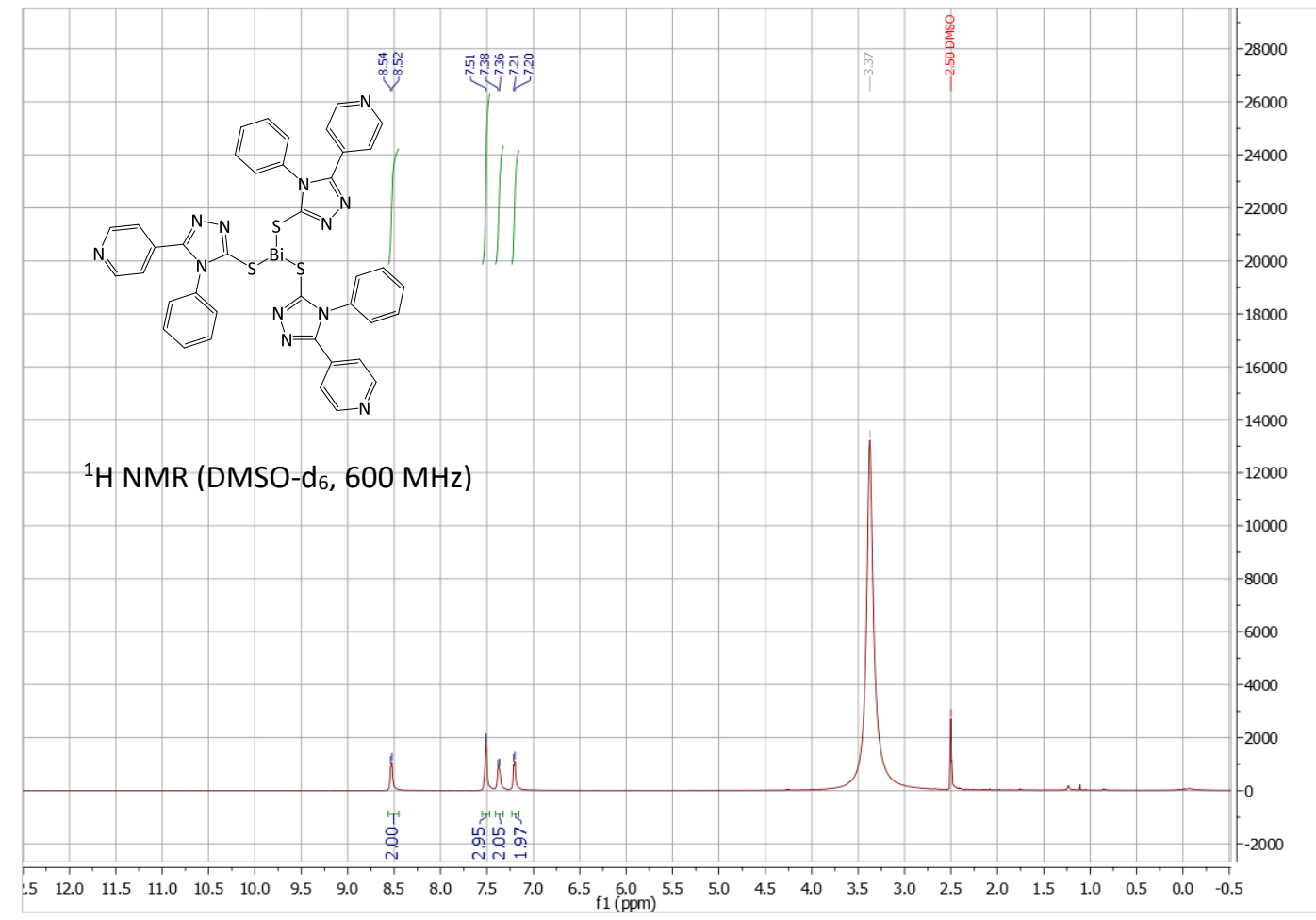

Figure S62. ${ }^{1} \mathrm{H}$ NMR of $\left[\mathrm{Bi}\left(\mathrm{C}_{13} \mathrm{H}_{9} \mathrm{~N}_{4} \mathrm{~S}\right)_{3}\right]$ 3g $\left(\mathrm{DMSO}_{-} \mathrm{d}_{6}\right)$

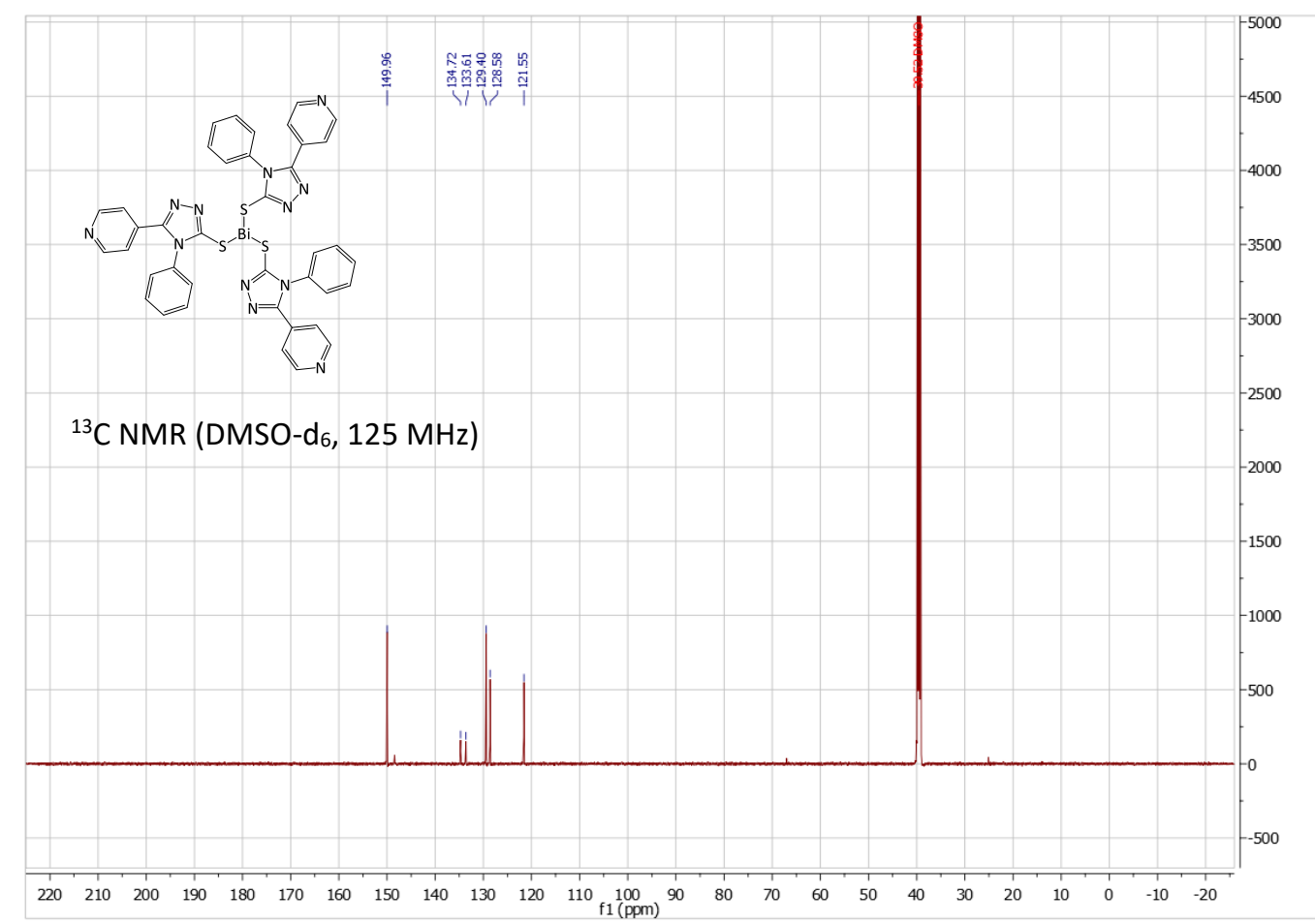

Figure S63. ${ }^{13} \mathrm{C}$ NMR of $\left[\mathrm{Bi}\left(\mathrm{C}_{13} \mathrm{H}_{9} \mathrm{~N}_{4} \mathrm{~S}\right)_{3}\right] \mathbf{3 g}\left(\mathrm{DMSO}-\mathrm{d}_{6}\right)$ 


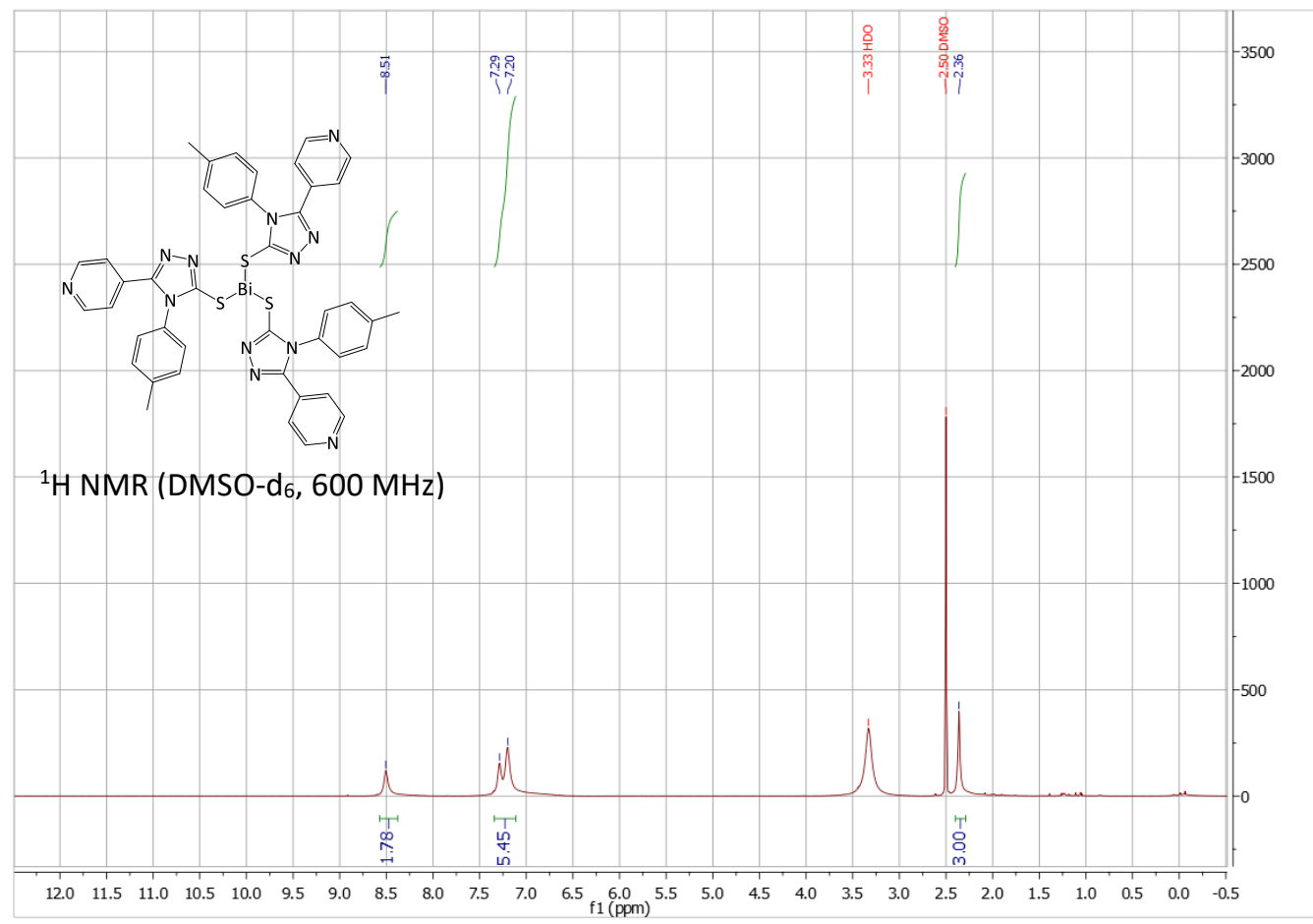

Figure S64. ${ }^{1} \mathrm{H}$ NMR of $\left[\mathrm{Bi}\left(\mathrm{C}_{14} \mathrm{H}_{11} \mathrm{~N}_{4} \mathrm{~S}\right)_{3}\right]$ 3h (DMSO-d $)$

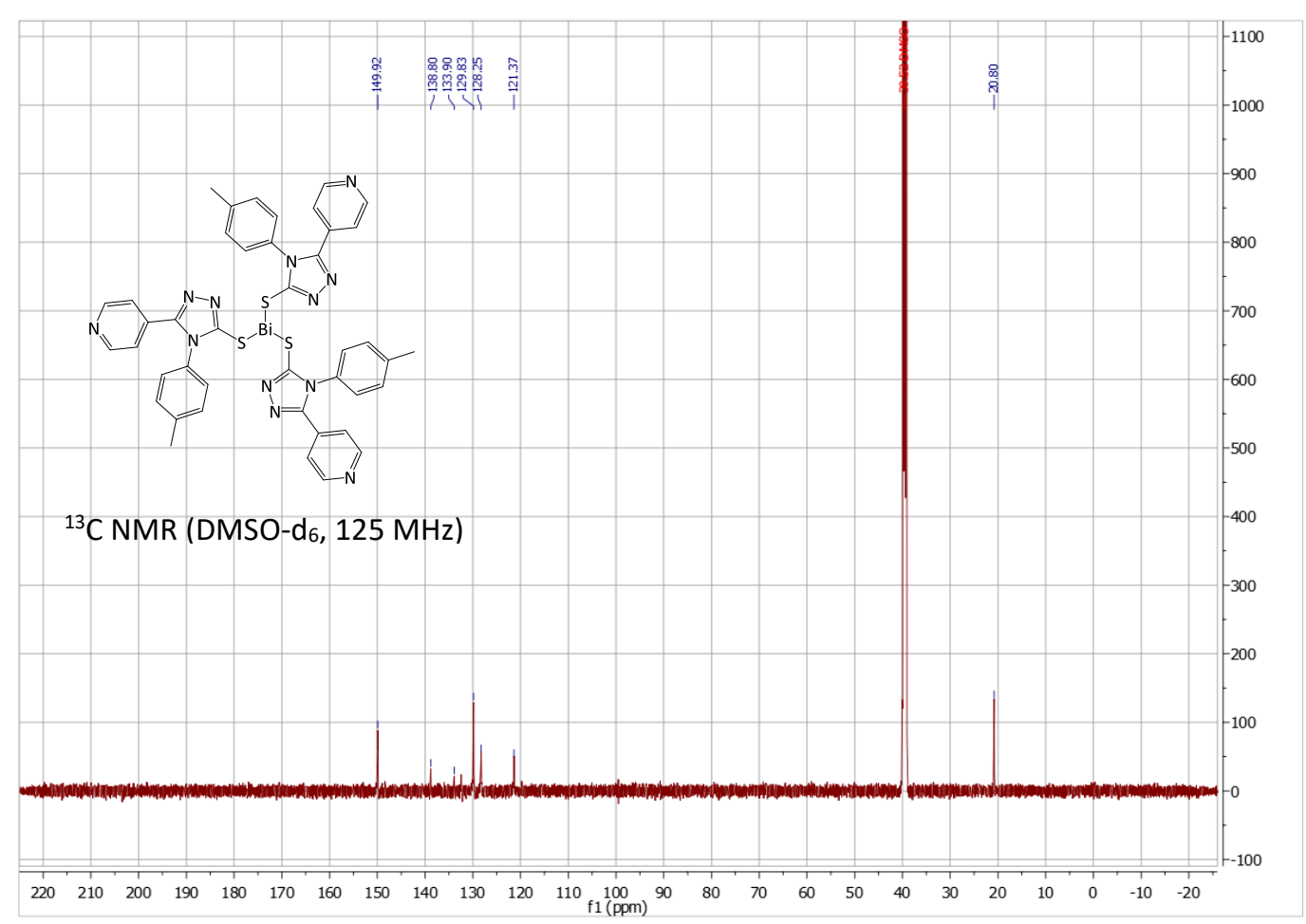

Figure S65. ${ }^{13} \mathrm{C}$ NMR of $\left[\mathrm{Bi}\left(\mathrm{C}_{14} \mathrm{H}_{11} \mathrm{~N}_{4} \mathrm{~S}\right)_{3}\right]$ 3h $\left(\mathrm{DMSO}-\mathrm{d}_{6}\right)$ 


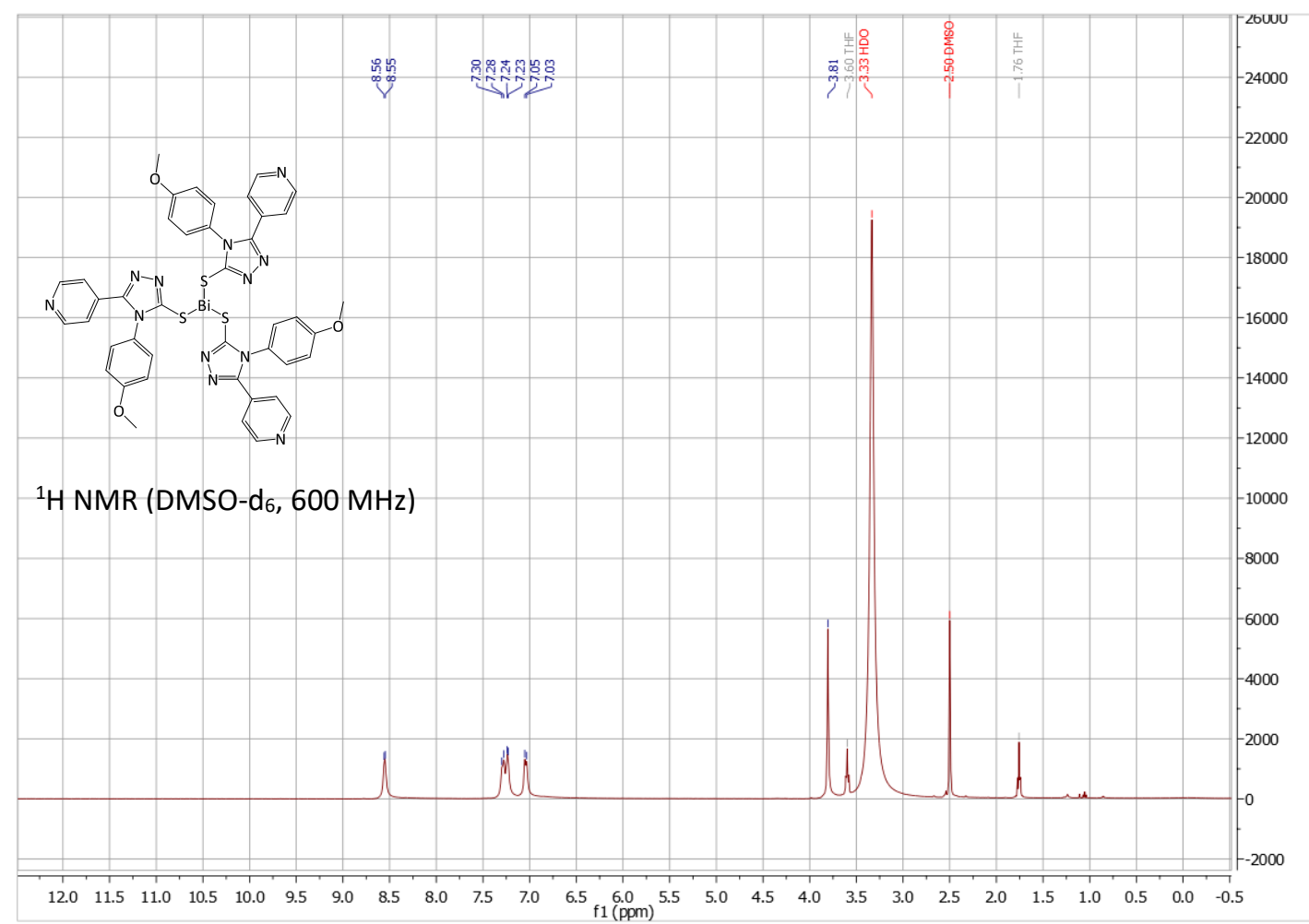

Figure S66. ${ }^{1} \mathrm{H}$ NMR of $\left[\mathrm{Bi}\left(\mathrm{C}_{14} \mathrm{H}_{11} \mathrm{~N}_{4} \mathrm{OS}\right)_{3}\right]$ 3i $\left(\mathrm{DMSO}^{-} \mathrm{d}_{6}\right)$

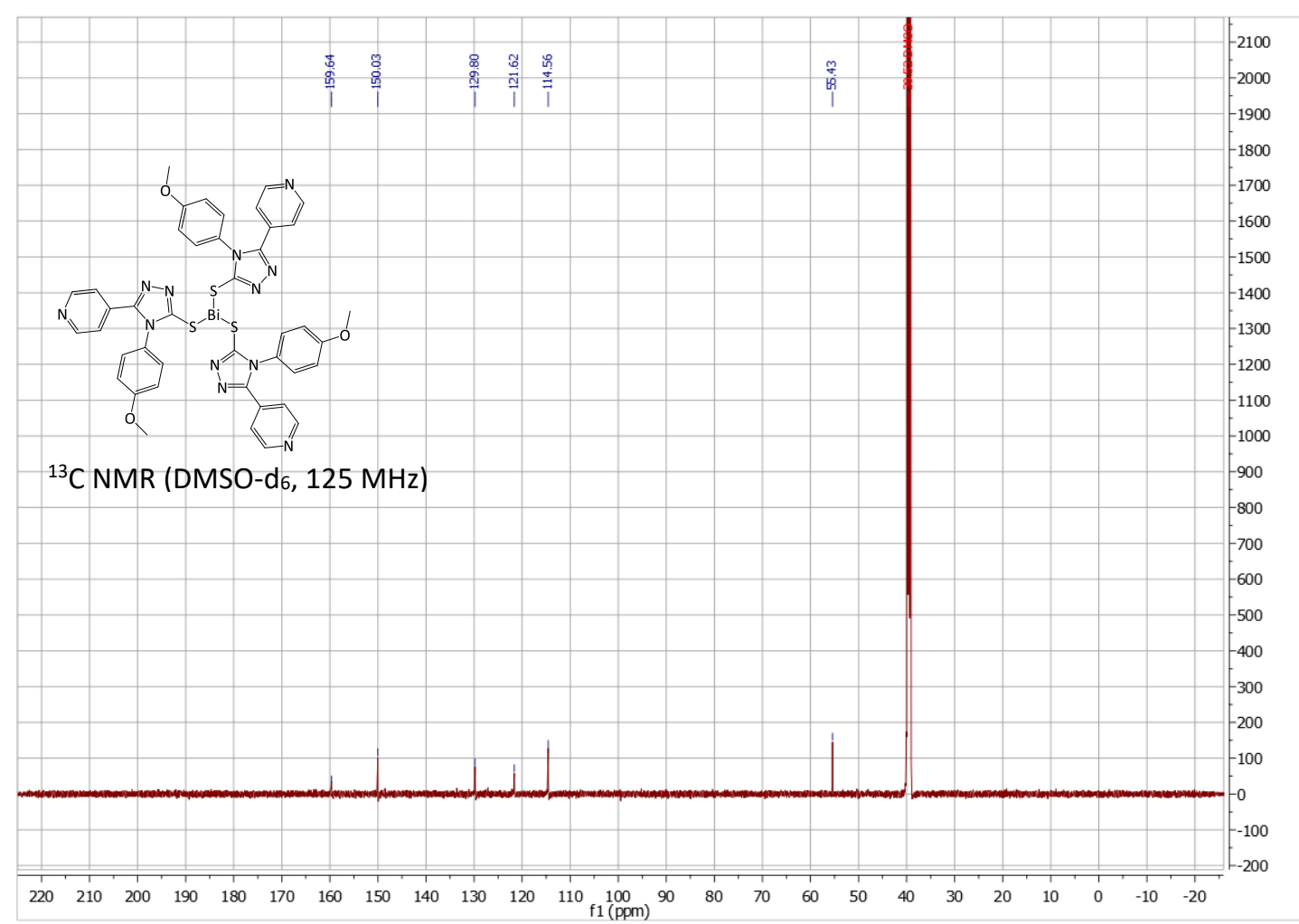

Figure S67. ${ }^{13} \mathrm{C}$ NMR of $\left[\mathrm{Bi}\left(\mathrm{C}_{14} \mathrm{H}_{11} \mathrm{~N}_{4} \mathrm{OS}\right)_{3}\right] 3 \mathbf{3 i}\left(\mathrm{DMSO}-\mathrm{d}_{6}\right)$ 


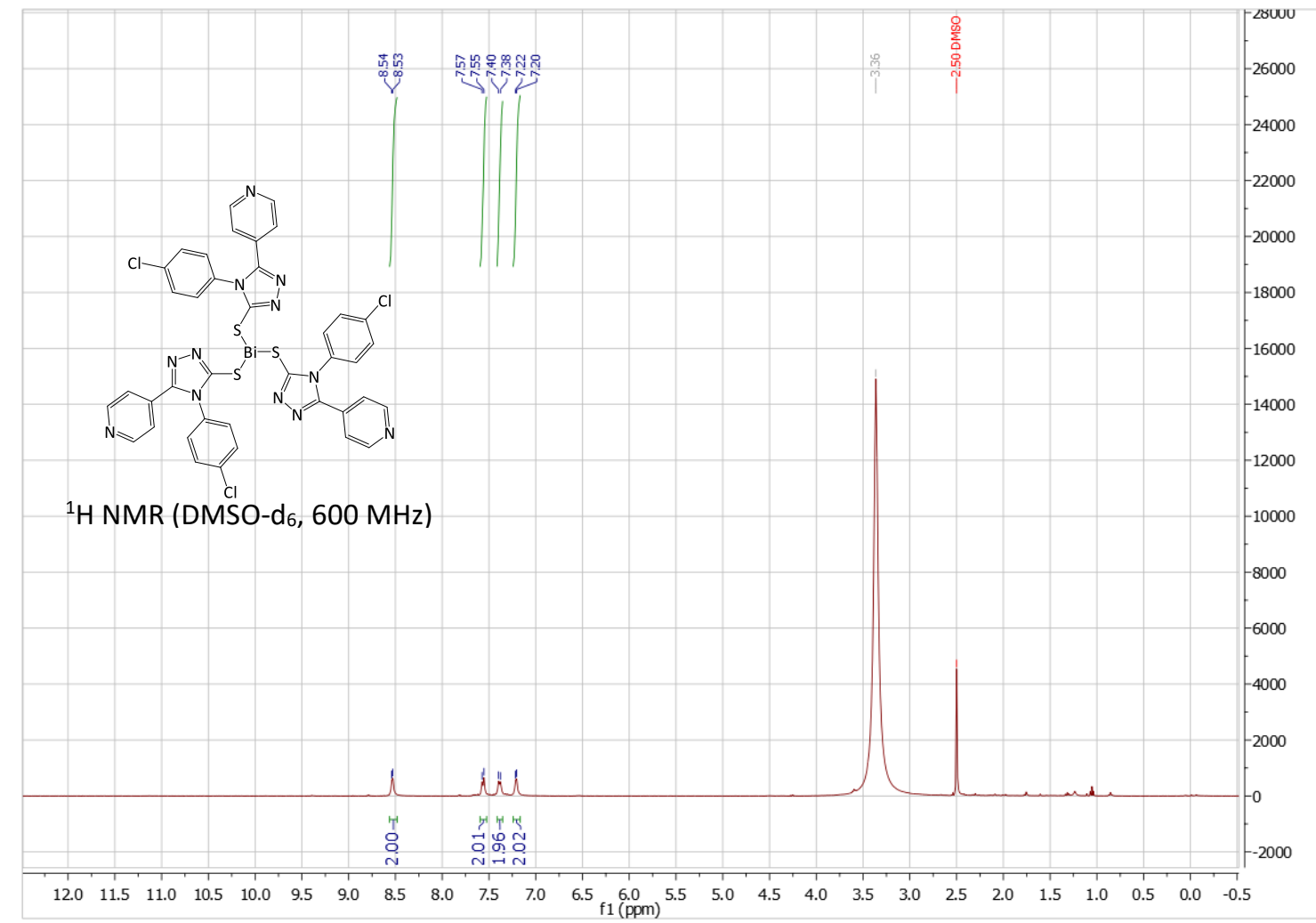

Figure S68. ${ }^{1} \mathrm{H}$ NMR of $\left[\mathrm{Bi}\left(\mathrm{C}_{13} \mathrm{H}_{8} \mathrm{~N}_{4} \mathrm{ClS}\right)_{3}\right] \mathbf{3 j}\left(\mathrm{DMSO}-\mathrm{d}_{6}\right)$

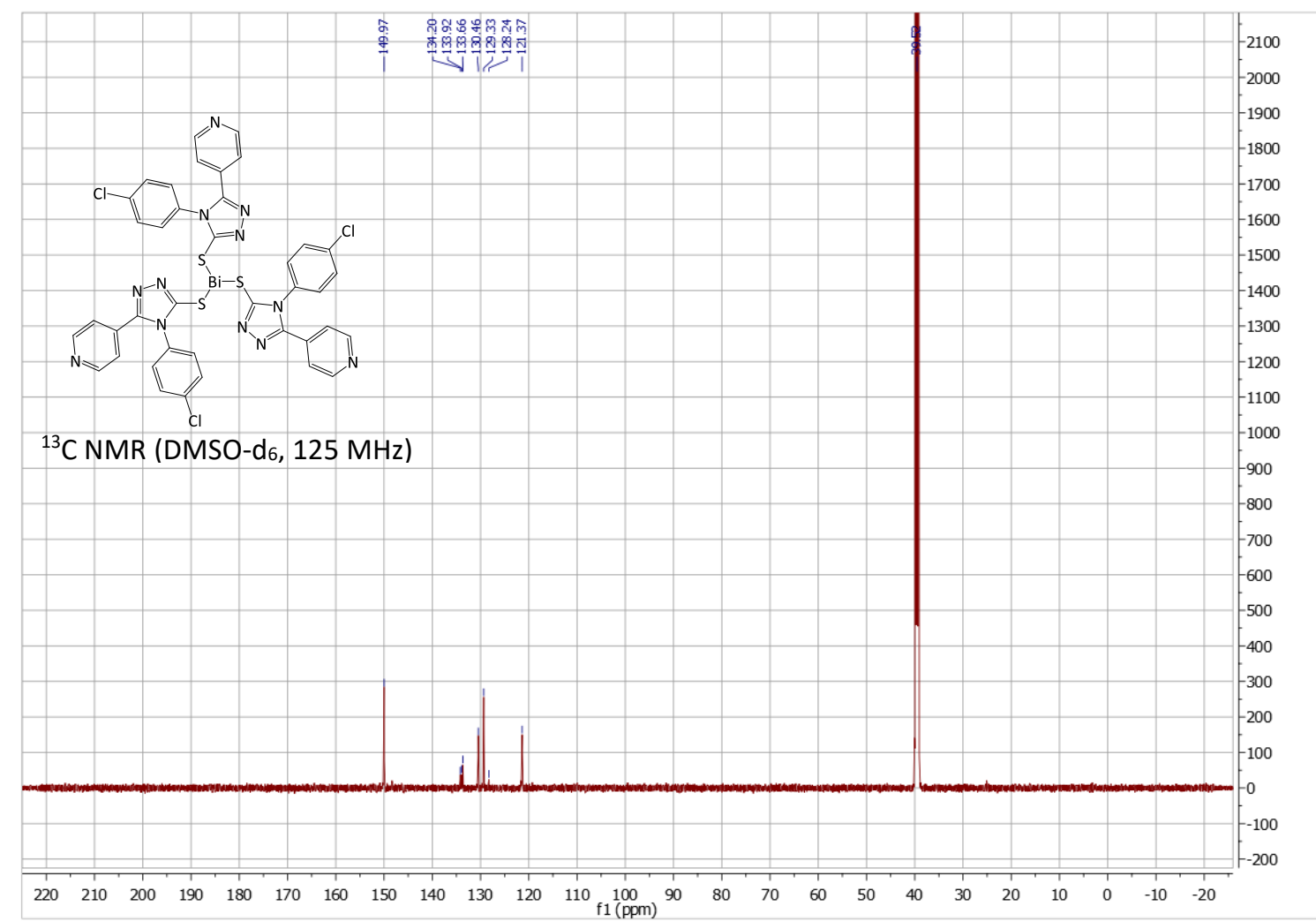

Figure S69. ${ }^{13} \mathrm{C}$ NMR of $\left[\mathrm{Bi}\left(\mathrm{C}_{13} \mathrm{H}_{8} \mathrm{~N}_{4} \mathrm{ClS}\right)_{3}\right] \mathbf{3 j}\left(\mathrm{DMSO}-\mathrm{d}_{6}\right)$ 


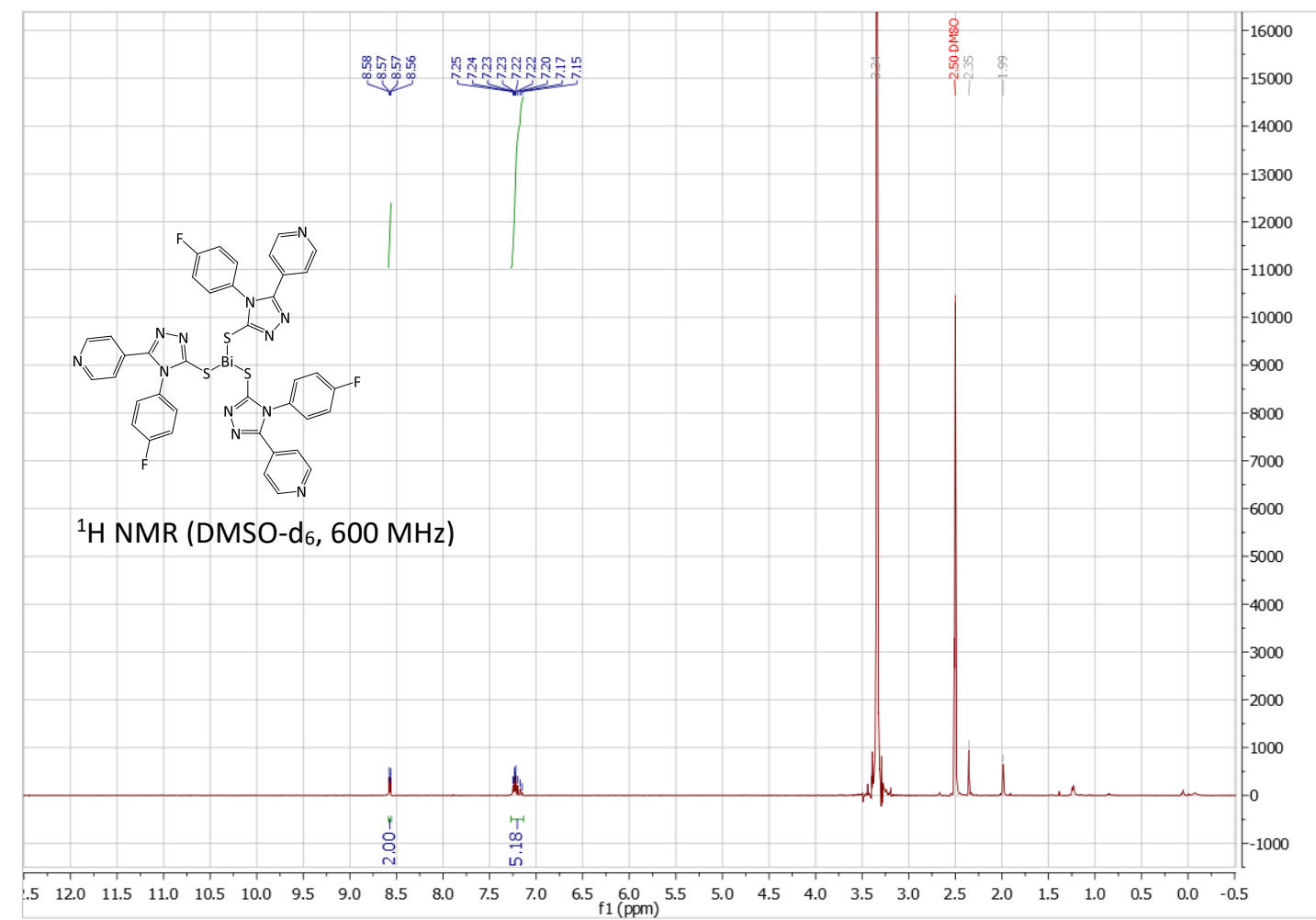

Figure S70. ${ }^{1} \mathrm{H}$ NMR of $\left[\mathrm{Bi}\left(\mathrm{C}_{13} \mathrm{H}_{8} \mathrm{~N}_{4} \mathrm{FS}\right)_{3}\right] \mathbf{1 k}\left(\mathrm{DMSO}-\mathrm{d}_{6}\right)$

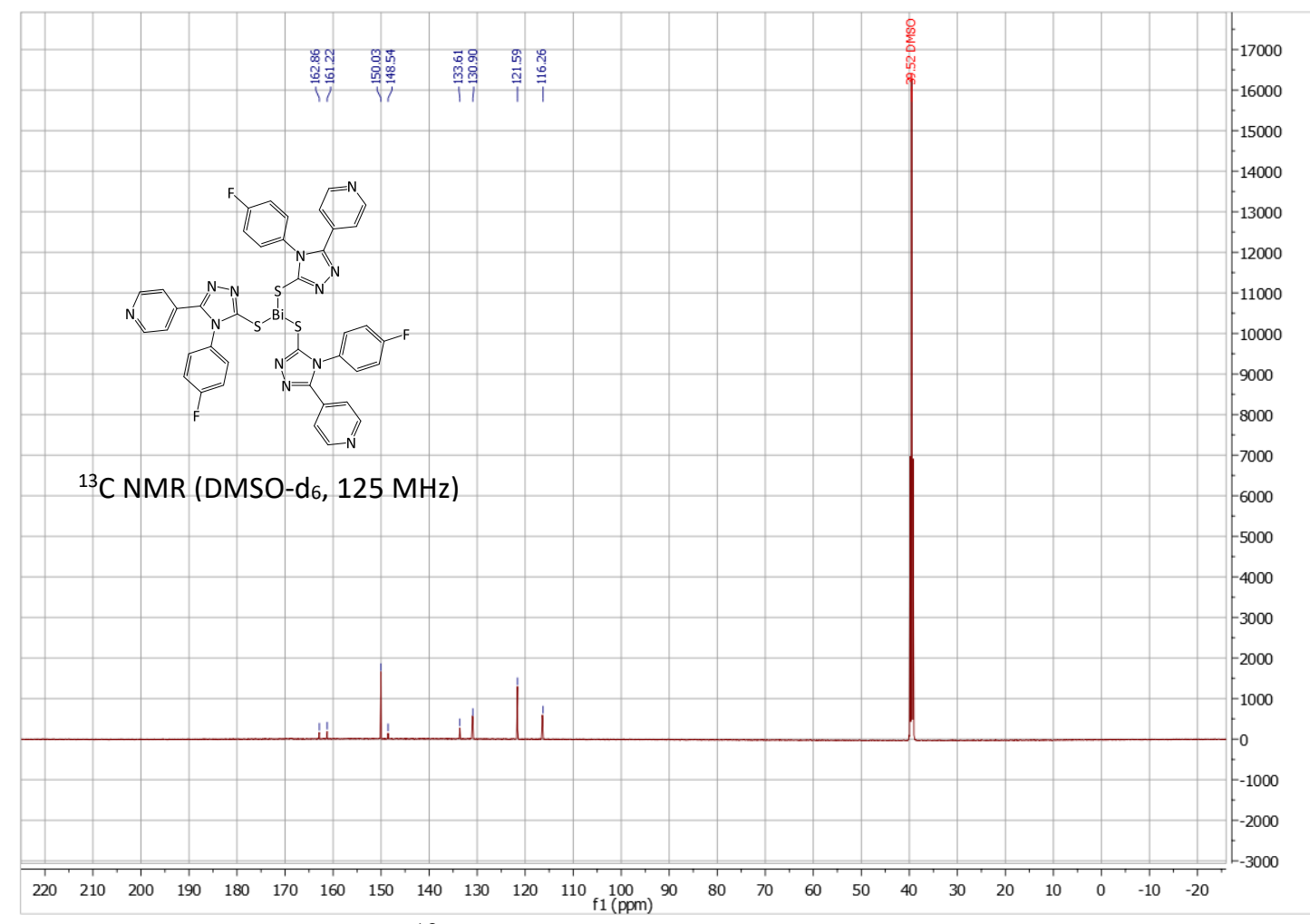

Figure S71. ${ }^{13} \mathrm{C}$ NMR of $\left[\mathrm{Bi}\left(\mathrm{C}_{13} \mathrm{H}_{8} \mathrm{~N}_{4} \mathrm{FS}\right)_{3}\right] 1 \mathbf{k}\left(\mathrm{DMSO}-\mathrm{d}_{6}\right)$ 


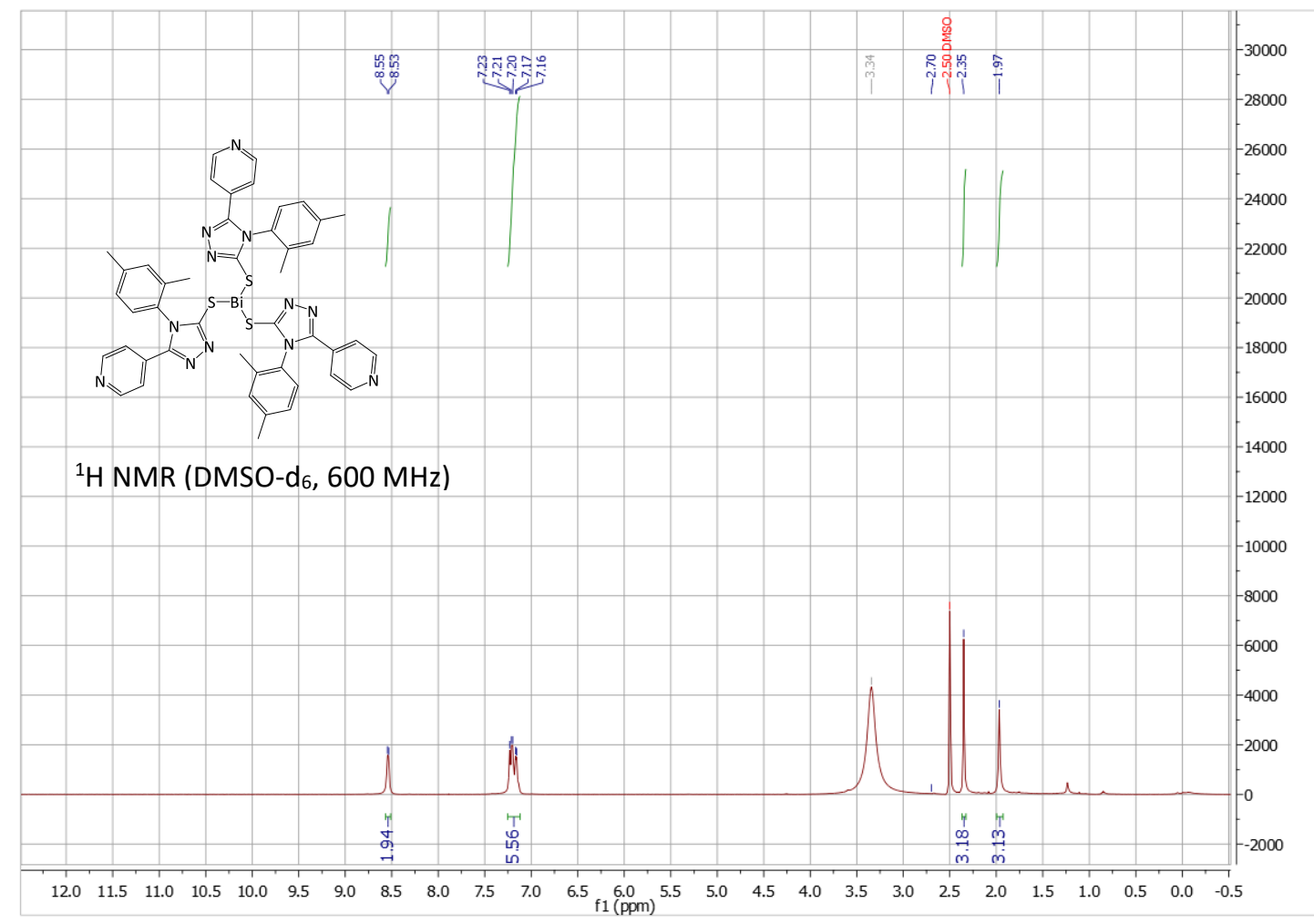

Figure S72. ${ }^{1} \mathrm{H} \mathrm{NMR}$ of $\left[\mathrm{Bi}\left(\mathrm{C}_{15} \mathrm{H}_{13} \mathrm{~N}_{4} \mathrm{~S}\right)_{3}\right] 11\left(\mathrm{DMSO}-\mathrm{d}_{6}\right)$

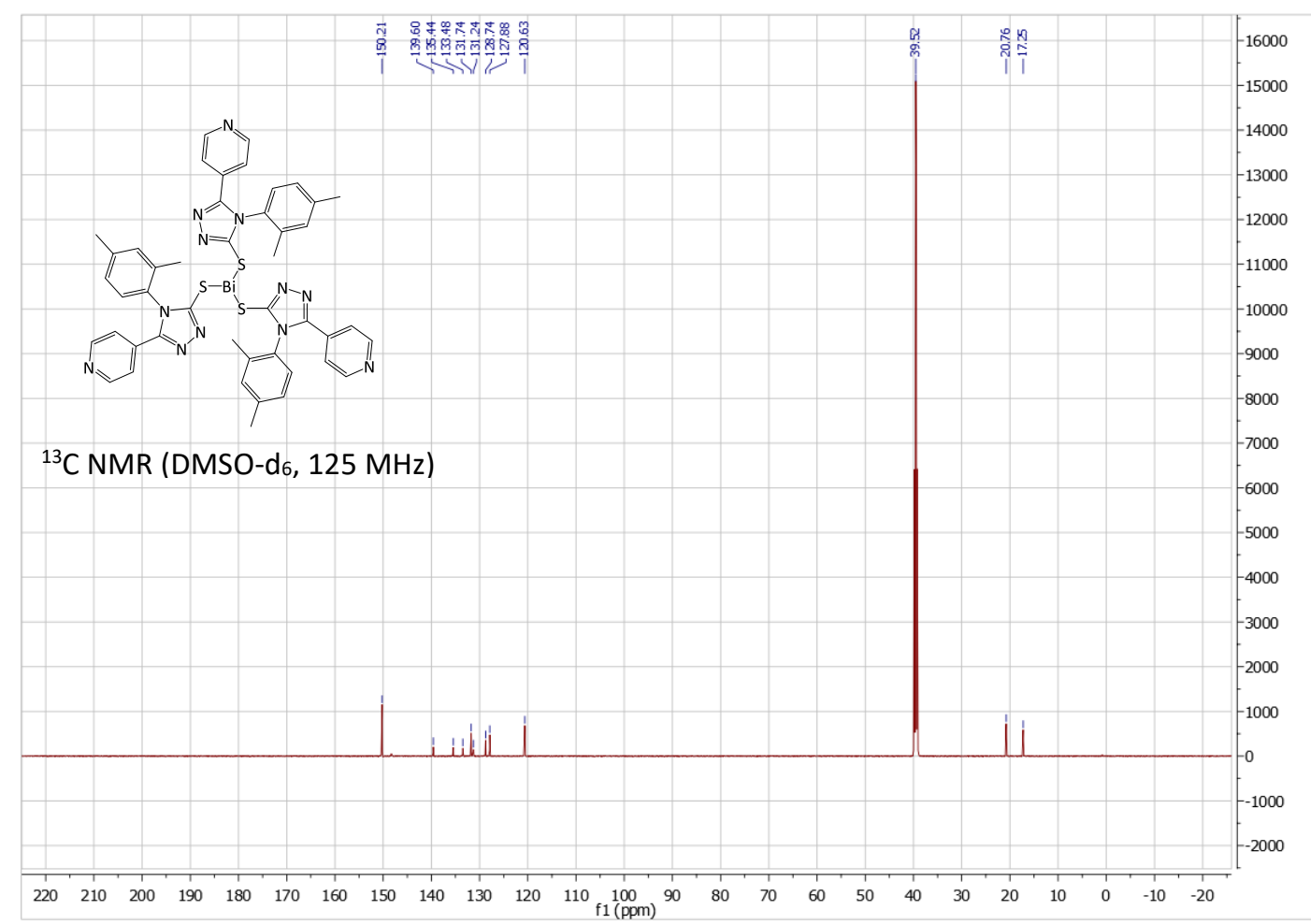

Figure S73. ${ }^{13} \mathrm{C} \mathrm{NMR}$ of $\left[\mathrm{Bi}\left(\mathrm{C}_{15} \mathrm{H}_{13} \mathrm{~N}_{4} \mathrm{~S}\right)_{3}\right] \mathbf{1 l}\left(\mathrm{DMSO}-\mathrm{d}_{6}\right)$

\section{References}

(1) Megally Abdo, N. Y.; Kamel, M. M. Synthesis and Anticancer Evaluation of 1,3,4- 
Oxadiazoles, 1,3,4-Thiadiazoles, 1,2,4-Triazoles and Mannich Bases. Chem. Pharm. Bull. 2015, 63, 369-376. 